\title{
The Internet of Bodies: A Systematic Survey on Propagation Characterization and Channel Modeling
}

\author{
Abdulkadir Celik, Senior Member, IEEE, Khaled N. Salama, Senior Member, IEEE, \\ and Ahmed M. Eltawil, Senior Member, IEEE.
}

\begin{abstract}
The Internet of Bodies (IoB) is an imminent extension to the vast Internet of things domain, where interconnected devices (e.g., worn, implanted, embedded, swallowed, etc.) located in-on-and-around the human body form a network. Thus, the IoB can enable a myriad of services and applications for a wide range of sectors, including medicine, safety, security, wellness, entertainment, to name but a few. Especially considering the recent health and economic crisis caused by novel coronavirus pandemic, a.k.a. COVID-19, the IoB can revolutionize today's public health and safety infrastructure. Nonetheless, reaping the full benefit of IoB is still subject to addressing related risks, concerns, and challenges. Hence, this survey first outlines the IoB requirements and related communication and networking standards. Considering the lossy and heterogeneous dielectric properties of the human body, one of the major technical challenges is characterizing the behavior of the communication links in-on-and-around the human body. Therefore, this paper presents a systematic survey of channel modeling issues for various link types of human body communication (HBC) channels below 100 $\mathrm{MHz}$, the narrowband (NB) channels between $400 \mathrm{MHz}$ and 2.5 GHz, and ultra-wideband (UWB) channels from 3 to $10 \mathrm{GHz}$. After explaining bio-electromagnetics attributes of the human body, physical and numerical body phantoms are presented along with electromagnetic propagation tool models. Then, the first-order (i.e., path loss, shadowing, multipath fading) and the second-order (i.e., delay spread, power delay profile, average fade duration, level crossing rate, etc.) channel statistics for NB and UWB channels are covered with a special emphasis on body posture, mobility, and antenna effects. For the HBC channels, three different coupling methods are considered: capacitive, galvanic, and magnetic. Based on these coupling methods, four different channel modeling methods (i.e., analytical, numerical, circuit, and empirical) are investigated, and electrode effects are discussed. Lastly, interested readers are provided with open research challenges and potential future research directions.
\end{abstract}

Index Terms-Internet of things, Internet of bodies, bioelectromagnetics, phantoms, narrowband, ultra-wideband, capacitive, galvanic, human body communications, channel modeling, antennas, propagation.

\section{INTRODUCTION}

$\mathrm{T}$ HE Internet of things (IoT) is a technological revolution that integrates the physical and digital worlds by interconnecting uniquely identifiable smart objects [1]. The IoT targets at ubiquitous connectivity among anyone/anything at any place/time for any service over any network. The recent report of international data corporation forecasts that an estimated 22 billion IoT devices in 2018 are foreseen to reach 41.6 billion in 2025 , generating 79.4 zettabytes of dat: ${ }^{1}$

Authors are with Computer, Electrical, and Mathematical Sciences \& Engineering (CEMSE) Division at King Abdullah University of Science and Technology (KAUST), Thuwal, KSA 23955-6900.

${ }^{1}$ https://www.idc.com/getdoc.jsp?containerId=prUS45213219
These numbers and ambitious goals naturally propel IoT as a mega-trend in next-generation communication and information technologies (ICT). As enabler of such a holistic approach towards digitizing and connecting a plethora of devices, all legacy telecommunications and networking technologies have come under the umbrella of IoT such as cellular networks [2], satellite networks [3], optical networks [4], wireless sensor networks [5], body area networks [6], cognitive radio networks [7]-[9], wireless-fidelity (Wi-Fi) [10], light-fidelity (Li-Fi) [11], Bluetooth [12], ZigBee [13], LoRa [14], to name but a few. Therefore, the IoT era dictates a radical paradigm shift in our perception of almost all verticals, including business, industry, energy, media, education, public health and safety, transportation, and logistics. The IoT can be classified into 'Internet of X-Things' where $\mathrm{X}$ may stand for space [15], underground [16], underwater [17], industrial [18], wearable [19], defense and public safety [20], health-care [21], and so on. In this survey, we narrow the scope to the body-centric IoT, which will be referred to as 'Internet of Bodies' (IoB) throughout the text.

\section{A. A Taxonomy of IoB Devices}

The IoB is an imminent extension to the vast IoT domain, where connected devices located in-on-and-around the human body form a network to enable a myriad of applications. Although an early version of IoB was first conceptualized in the realm of wireless body area networks (WBANs) [22], their wide-spread use in today's daily life has become possible as a result of parallel advancements in microelectronics, wireless communications, and signal processing. The IoB devices can be worn, swallowed, implanted in the body, or even embedded into the skin. Smartwatches, fitness tracker rings/wrist-bands, wireless headphones, heads-up display glasses, virtual reality headsets, smart tattoos/bio-patches, and global positioning system (GPS) enabled shoes are examples of wearable IoB devices. Digital drug delivery pills and ingestible sensors (e.g., endoscopy capsules) are typical examples of swallowable IoB devices. For implantable IoB, cardioverter defibrillators and heart pacemakers are a case in point. Lastly, embedded IoB devices can be simply chips buried under the skin. A very cutting-edge instance is brain-computer interface technology, where an IoB device allows a real-time connection between the brain and remote computers for the purpose of monitoring and controlling [23]. It has also recently come into fashion to inject a large rice grain size microchip into the hand for the purpose of biometric identification and authorization grant [24]. 


\section{Internet of Bodies}

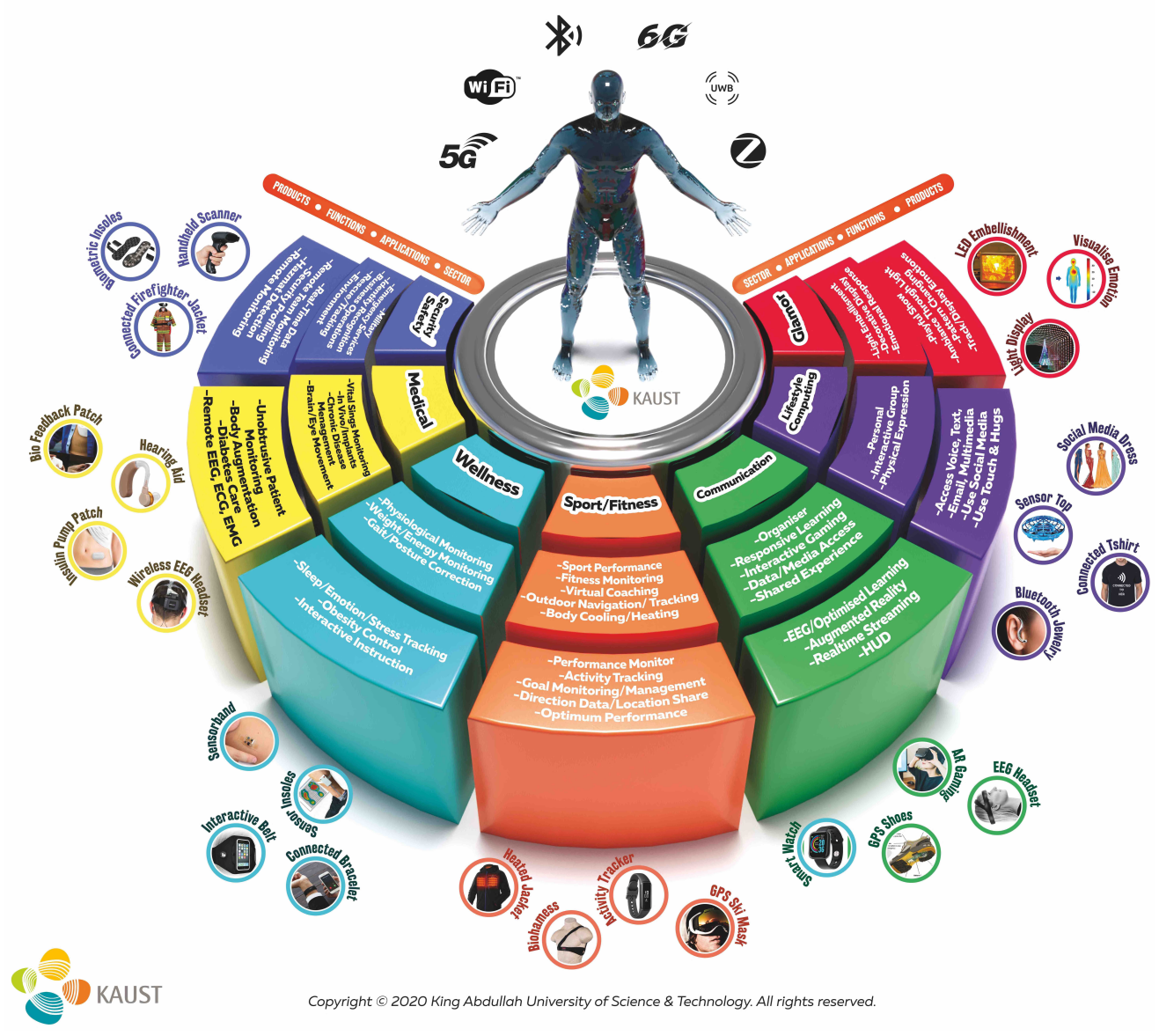

Fig. 1: A taxonomy of IoB Applications.

\section{B. A Taxonomy of IoB Applications}

As pictorially categorized in Fig. 1. IoB devices located in-on-and-around the human body enable many services for a wide range of sectors, each with various applications and functions. Among all these sectors, public health-care and safety require specific attention as world population growth poses three major challenges [25]: the demographic peak of baby boomers, the aging population as a result of the increasing life expectancy, and boosting health care expenditures. 2019 base estimates of the Organization for Economic Co-operation and Development (OECD) foresee health spending of member countries to reach $10.2 \%$ of Gross Domestic Product (GDP) by 2030, the highest $21.3 \%$ for the USA, up from $8.8 \%$ in 2015 [26]. These statistics mandate a dramatic shift towards a more scalable and affordable health-care system. More importantly, millions of people die from diabetes, obesity, cancer, cardiovascular diseases, asthma, and many more fatal or chronic diseases every single year. The majority of the current fatal diseases share one critical feature in common: most of the patients have the disease diagnosed a long while after they experience the early symptoms. Research has shown that early diagnosis and screening can control or even prevent most chronic diseases. Therefore, IoB can support future health-care systems for early detection and prevention of diseases through proactive wellness screening technologies. By enabling close and continuous monitoring, IoB can also be an effective tool for the rehabilitation of patients recovering after surgery or medication.

Indeed, all the statistics presented in the previous paragraph have to be reconsidered in the post-COVID-19 world. The COVID-19 is a respiratory virus that originated from the city of Wuhan, China, in December 2019. In March 2020, the World Health Organization (WHO) declared COVID-19 as a global pandemic. The WHO situation reports record almost 25 million infections and nearly a million deaths by September 1 , 2020 [27]. Governments have imposed severe restrictions to reduce the infection rates by minimizing social interaction so that the curve of hospitalized patients is flattened so as not to exceed the hospital capacity of the national health care system. In addition to the unprecedented humanitarian crisis caused by COVID-19, the World Bank has recently reported that the measurements taken across the globe have brought $92.9 \%$ of world countries into a 5\% global recession on average [28]. The World Bank also estimates 720 million population under 
extreme poverty $\left.\right|^{2}$ by 2021 , which is about $22 \%$ increase from 590 million estimate just after the spread of COVID-19. In this regard, IoB can be of great help in fighting pandemic diseases by detecting new cases based on physiological data and vital signs, remote monitoring of positive but asymptomatic patients in self-quarantine, and tracing other potential cases in contact with cases previously identified as positive. IoB based health monitoring is also a good solution to minimize the infection risk for health-care providers whose dedicated-selfless-andrelentless efforts have been proven priceless on the front-line against COVID-19.

\section{IoB Risks, Concerns, and Challenges}

Albeit, with all these fantastic opportunities, several risks, concerns, and challenges must still be addressed to realize the full potential of IoB. Security risks and privacy concerns are the major obstacles hindering the wide-spread use of IoB devices. This is especially crucial for IoB devices that have control over vital body functions (e.g. heart pace-makers)or gather sensitive data of users. Although laws and regulations exist regarding personal health information held by medical providers and insurance companies, these outdated regulations do not address technology companies that store and process sensitive information in their data centers. Focusing only on the "deemed" sensitivity of certain data items, does not necessarily address the ability to use non-sensitive data to infer sensitive information by means of data analytics.

There are also technical challenges in terms of contradictory design objectives such as safety, miniaturization, battery life, and communication performance. For example, an implant/embedded IoB device requires both a small form factor and long battery life. On the other hand, safety regulations limit the maximum transmission power to avoid harm to the human body, which also behaves as a delimiter on communication performance. As a lossy and heterogeneous dielectric medium, the communication channel's characterization in-onand-around the human body is not only non-trivial but also crucial to optimize overall communication performance. This is mainly because of the fact that the cross-layer optimization of the IoB network heavily depends on precise channel estimation. Noting that subsequent sections go over IoB requirements in detail, the focus of this survey will be on the channel modeling and their interwoven relations with risks, concerns, and challenges.

\section{Survey Contributions and Organization}

As tabulated in Table I], several magazine and survey papers covered different aspects of wireless body area networks in the last decade. In [30] and [31], authors present a short review of applications, devices, and challenges of wireless body area networks (WBANs). It is followed by several survey papers which handle WBANs in a layer-by-layer fashion [6], [22], [32], [36], [37]. Since these works cover a wide range of WBAN related issues, they do not provide readers with an

\footnotetext{
${ }^{2}$ Extreme poverty is measured as the number of people living on less than 1.90 US dollars per day.
}

TABLE I: Related works

\begin{tabular}{|c|c|c|c|}
\hline Year & Ref. & Type & Content \& Style \\
\hline 2007 & 29 & Magazine & Antennas \& propagation for on-body WBAN \\
\hline 2009 & 30 & Magazine & Principles and challenges of $\mathrm{HBCs}$ \\
\hline 2009 & $31]$ & Magazine & $\begin{array}{l}\text { A review of WBAN applications, } \\
\text { devices, and radio technologies }\end{array}$ \\
\hline 2011 & {$[\overline{6}]$} & Survey & A layer-by-layer survey of WBAN \\
\hline 2011 & 22 & Survey & A layer-by-layer survey of WBAN \\
\hline 2012 & 32 & Survey & A layer-by-layer survey of WBAN \\
\hline 2013 & 33 & Magazine & Propagation models for in-body WBAN \\
\hline 2013 & $\overline{34}$ & Magazine & Propagation models for on-body WBAN \\
\hline 2013 & 35 & Survey & A survey on intrabody communications \\
\hline 2014 & $\overline{36}$ & Survey & A layer-by-layer survey of WBAN \\
\hline 2014 & 37] & Survey & A survey on technologies \& design challenges \\
\hline 2017 & 38 & Survey & A review on $\mathrm{HBC}$ \\
\hline 2018 & 39 & Survey & A survey of galvanic coupling HBC \\
\hline
\end{tabular}

in-depth insights into the channel modeling issues. On the other hand, the in-body channel characterization is reviewed in [33], which is followed by on-body channel characterization in [29] and [34]. The surveys on human body communication is relatively more recent and can be found in [38] and [39].

To the best of authors' knowledge, this survey is the first to provide a systematic survey on modeling narrowband, ultrawideband, and human body communication channels in-onand-around the human body. In previous surveys, channel characteristics was either briefly mentioned or a specific channel type was considered (e.g., in-body or on-body). We are also the first to present the IoB concept as a holistic view along with its requirements and related standards. As can be seen from the organization chart of the survey [c.f. Fig. 2], our purpose with this comprehensive survey is to provide researchers with reference material, from which they can acquire a holistic view on channel characterization issues to realize future IoB technologies.

\section{IOB REQUIREMENTS AND RELATED STANDARDS}

Standardizing the IoB communications and networking is a challenging task due to the broad range of IoB applications presented in Section I-B The standardizations bring many benefits including reduced research and development costs thanks to the publicly available solid technical specifications and guidelines; compatibility and interoperability between products manufactured by different vendors; rapid and broad spread of the technology. Therefore, this section first outlines the main $\mathrm{IoB}$ requirements before delving into the various entities' standardization efforts.

\section{A. IoB Requirements}

In this subsection, we briefly summarize main IoB requirements in light of the application types discussed above.

1) Quality of Service $(Q o S)$ : The QoS demands of IoB applications can significantly deviate from each other in terms of data rate, bit error rate (BER), latency, and reliability. The data rate needs may range from Kbps (e.g, glucose monitoring: < $1 \mathrm{Kbps}$, drug delivery: < $16 \mathrm{Kbps}$, EEG: $86.4 \mathrm{Kbps}$, voice: 50-100 Kbps, ECG: $192 \mathrm{Kbps}$ ) to Mbps (e.g., capsule endoscopy and audio streaming: $1 \mathrm{Mbps}$, EMG: $1.5 \mathrm{Mbps}$, video streaming: 10-100 Mbps) [37]. While medical and military 


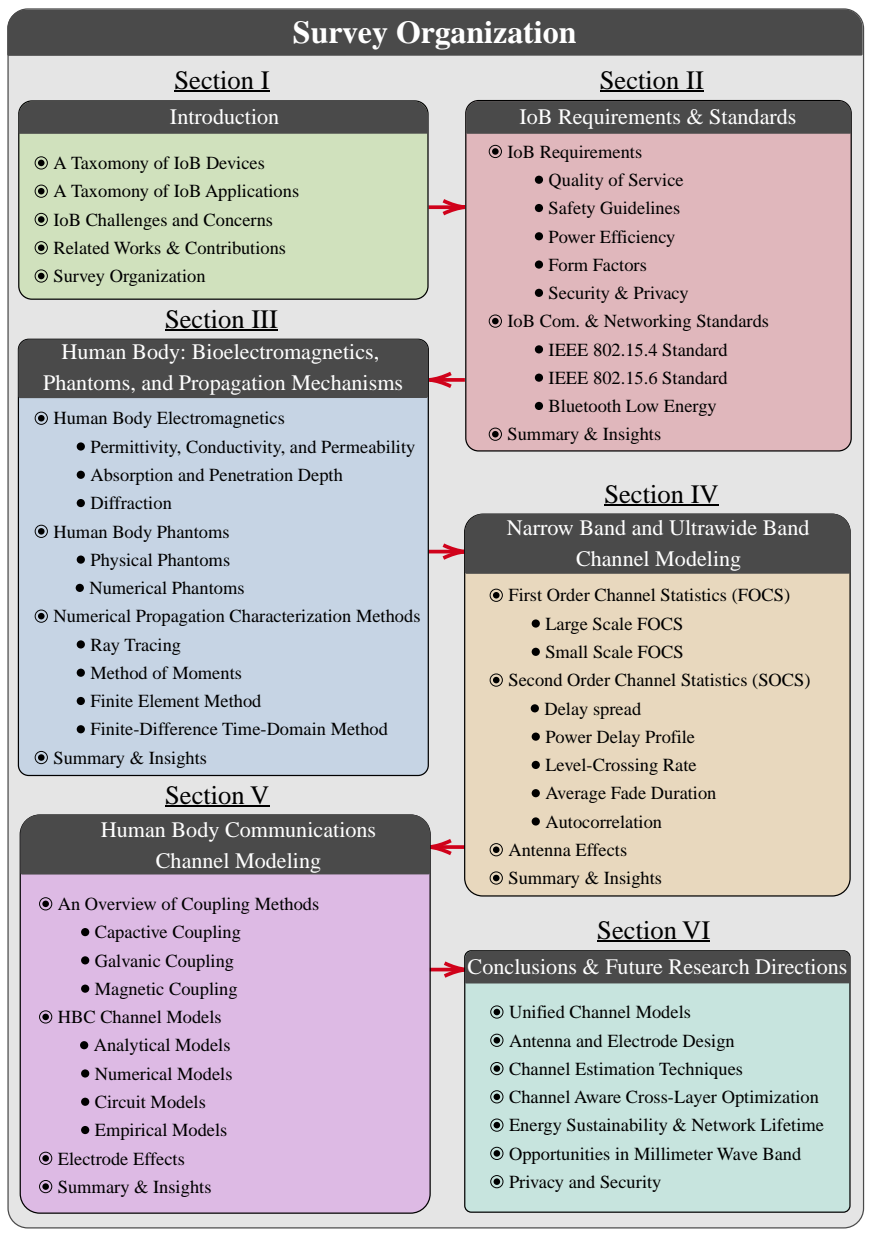

Fig. 2: Schematic illustration of the survey organization.

applications have stringent BER demands $\left(<10^{-10}\right)$, multimedia applications are relatively more tolerable $\left(<10^{-5}\right)$. Since medical and military applications generally fall within the scope of ultra-reliable low-latency communications (URLLC) class, IoB network architecture should be designed to provide a diverse range of QoS demands by prioritizing traffic types based on urgency and criticality of the underlying applications.

At this point, the coexistence of IoB nodes and other wireless technologies has significant impacts on meeting the QoS demands. Generic IoT devices are generally designed to operate on license-free globally available ISM bands, which are already overcrowded by other wireless standards, including IEEE 802.11 (Wi-Fi) [10], IEEE 802.15.1 (WPAN) [40], IEEE 802.15.4 (ZigBee) [41], and Bluetooth [12]. The coexistence of IoBs and these standards are addressed in [42]-[44]. Considering the ever-increasing IoT devices operating on ISM bands, it is hardly possible to guarantee URLLC for critical IoB applications. Therefore, the physical layer (PHY) should employ appropriate interference avoidance and error correction methods to improve BER performance. Moreover, the PHY should also be supported by higher layers to improve packet loss rate and transmission delays with effective collision and congestion avoidance techniques.

2) Safety Guidelines: In the context of wireless communications, the primary health concern is mainly heating of body tissues since the energy of transmitted electromagnetic fields is absorbed by the body. The degree of heating effects due to the overexposure to electromagnetic fields depends on the operating frequency, signal intensity, the duration of exposure, the location of exposure on the human body, the distance from the transmitter as well as other factors such as shielding. General safety guidelines are specified by The International Commission on Non-Ionizing Radiation Protection (ICNIRP) to limit time-varying electromagnetic field exposure on the human body [45]. For frequencies up to $10 \mathrm{GHz}$, these restrictions are determined in terms of Specific Absorption Rate (SAR), which is expressed in units of watts per kilogram $[\mathrm{W} / \mathrm{Kg}]$. Thus, the SAR represents the mass normalized energy rate coupled to biological tissues. Indeed, typical low power wireless devices do not radiate enough power to be a concern for whole-body SAR. However, since IoB devices are located in or on the human body, extra attention must be paid on localized SAR measured around the transceivers in/on the human body. Therefore, IoB related standards must comply with the minimum local SAR requirements of global (ICNRP [45]) or regional regulation entities, e.g., Scientific Committee on Emerging and Newly Identified Health Risks (SCENIHR) for European Union [46] and Federal Commission on Communications (FCC) for USA [47].

3) Power Efficiency: IoB nodes are specially designed as ultra-low-power communication devices for two main reasons: 1) To comply with the safety regulations mentioned above and 2) To maximize the network lifetime since IoB devices are typically battery powered. In particular, implanted and embedded IoB nodes require 5 years of battery lifetime as they are typically placed in the human body through a surgery process [48]. Likewise, wearable devices and on-body sensors are also preferred to have a lifetime of weeks or months for the sake of user satisfaction and maintenance easiness. A crosslayer optimization is necessary for an energy efficient system design, which is discussed in the subsequent sections of the survey.

4) Form Factor: IoB nodes generally have stringent form factor constraints that require squeezing antenna/electrode and battery into a tiny case while meeting the QoS demands of the application of interest. While miniaturization is crucial for implantable devices, flexibility and stretchability are more important comfort considerations for wearable devices [49]. Advances in stretchable electronics is a key technology to realize flexible wearable devices; e.g., liquid and microfluidic antennas [50], [51], displays [22], solar cells [52], battery [53], sensors [54], etc. Ink-jet printable textile electronics can also realize highly effective small form-factor wearable devices [55], [56]. Notice that antenna/electrode design for on/in-body devices is quite distinct due to the impacts of the human body on the radiation and polarization characteristics, which is discussed in more detail through Section IV-C and Section $\mathrm{V}-\mathrm{C}$

5) Security, Confidentiality, and Privacy: Most IoB applications require a high level of security, confidentiality, and privacy. However, this is a challenging task given the limited resources of energy, memory, and computational power. First of all, a trusted coordinator node is necessary for the manage- 


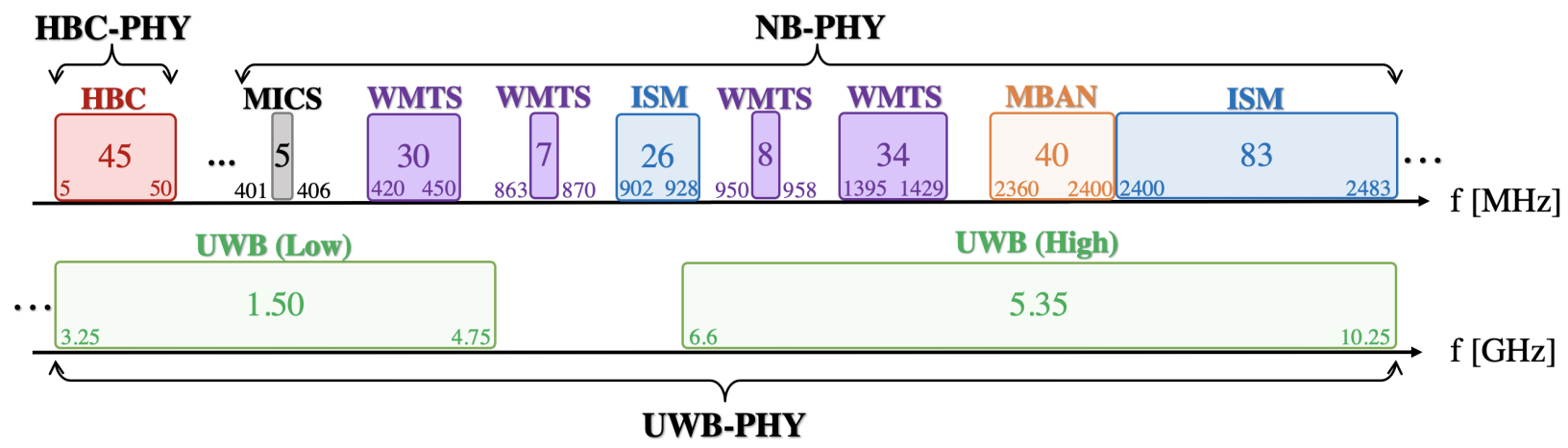

Fig. 3: Frequency bands of different PHYs defined by the IEEE 802.15.6 standard.

ment of adding/dropping nodes to/from the network as well as providing key distribution for encryption and decryption. The coordinator and IoB nodes also need to authenticate that data is received from a trusted pair, which can be done by computing a message authentication code based on a shared secret key. The integrity of data should also be protected against the adversary nodes' capabilities of altering original data. Furthermore, the confidentiality of user data must be carefully guarded against eavesdropping. Data integrity and privacy are especially important if the underlying application is sensitive to the age of information (e.g., data freshness). Since ensuring all these functions inevitably degrades other QoS metrics, the use of biometrics can help significantly reduce the complexity and its associated costs [57], [58]. The biometrics include heartbeat timing, fingerprint, voice, palm print and veins, face/iris recognition, gait, typing rhythm, etc. Unlike the license-free crowded RF bands, human body communications (HBC) offers extra levels of physical layer security by using the human body as a communication medium, which is also discussed in the subsequent sections of the survey.

\section{B. IoB-Related Communications and Networking Standards}

In this subsection, we summarize the main features of IEEE 802.15.4, IEEE 802.15.6, and Bluetooth standards. Although they are developed for WBANs, we believe they provide a good starting point for standardization of the IoB networks.

1) IEEE 802.15.4 Standard: The IEEE 802.15.4 standardizes the PHY and MAC specifications to support low cost, low power, low range, and low bit rate requirements of wireless personal area networks (WPANs) [41]. The upper layer operations of The IEEE 802.15.4 are defined by the ZigBee protocol of ZigBee Alliance [13] and 6LowPAN of the Internet engineering task force (IETF) [59]. The ZigBee protocol suite is later extended to ZigBee Pro for non-routing nodes with extra features of route aggregation, asymmetric link handling, conflict resolution, etc.

The IEEE 802.15.4 specifies a total of 27 half-duplex channels: a single channel with $20 \mathrm{Kbps}$ rate at $868 \mathrm{MHz}$ band, ten channels each with $40 \mathrm{Kbps}$ at $915 \mathrm{MHz}$ band, and sixteen channels each with $250 \mathrm{Kbps}$ at $2.45 \mathrm{GHz}$ band. In the MAC layer, beacon-enabled and non-beacon-enabled channel access modes are available. While the former employs the Carrier Sense Multiple Access/Collision Avoidance (CSMA/CA) algorithm with back-off periods, the later uses an unslotted CSMA/CA. Although an encryption algorithm is defined to cipher the transmitted data, key management and authentication policies are not specified. However, ZigBee manages these issues in the network and application layers by using advanced encryption standard (AES) with 128-bit key lengths.

2) IEEE 802.15.6 Standard: Unlike the IEEE 802.15.4 standard, the IEEE 802.15.6 standard is especially developed to meet the aforementioned distinctive QoS demands of WBANs [60]. As illustrated in Fig. 3, the IEEE 802.15.6 standard defines three different PHYs to address the breadth of WBAN applications:

a) HBC-PHY operates with a central frequency at $21 \mathrm{MHz}$ and a bandwidth of $5.25 \mathrm{MHz}$, which may support data rates ranging from $164 \mathrm{Kbps}$ to $1.3 \mathrm{Mbps}$.

b) NB-PHY requires NB-WBAN nodes to operate in at least one of the following bands: medical implant communication service (MICS) band on 402-405 MHz; wireless medical telemetry system (WMTS) bands on 420-450 $\mathrm{MHz}, 863-870 \mathrm{MHz}, 950-958 \mathrm{MHz}$, and 1395-1429 $\mathrm{MHz}$; medical body area network (MBAN) band on 2360$2400 \mathrm{MHz}$; and industrial-scientific-and-medical (ISM) bands on 902-908 MHz and 2400-2483.5 MHz. NB-PHY allows several bit rates ranging from $50 \mathrm{Kbps}$ to $1 \mathrm{Mbps}$ by using Bose-Chaudhuri-Hocquenghem $(\mathrm{BCH})$ error correction codes with basic modulation schemes. For example, Gaussian minimum shift keying (GMSK), $\pi / 2$ shifted differential binary phase-shift keying (DBPSK), and $\pi / 4$-shifted differential quadrature phase-shift keying (DQPSK).

c) UWB-PHY consists of low (3.25-4.75 GHz) and high $(6.60-10.25 \mathrm{GHz})$ bands, each of which is further divided into $500 \mathrm{MHz}$ sub-bands. The UWB technique is known with its ability to support either low rates over highly attenuated channels or high rates over short ranges. In addition to power-spectral density, its fine granularity time resolution is suitable for real-time applications [61]. The UWB-PHY provides high data rates $(0.2-15 \mathrm{Mbps})$ by 


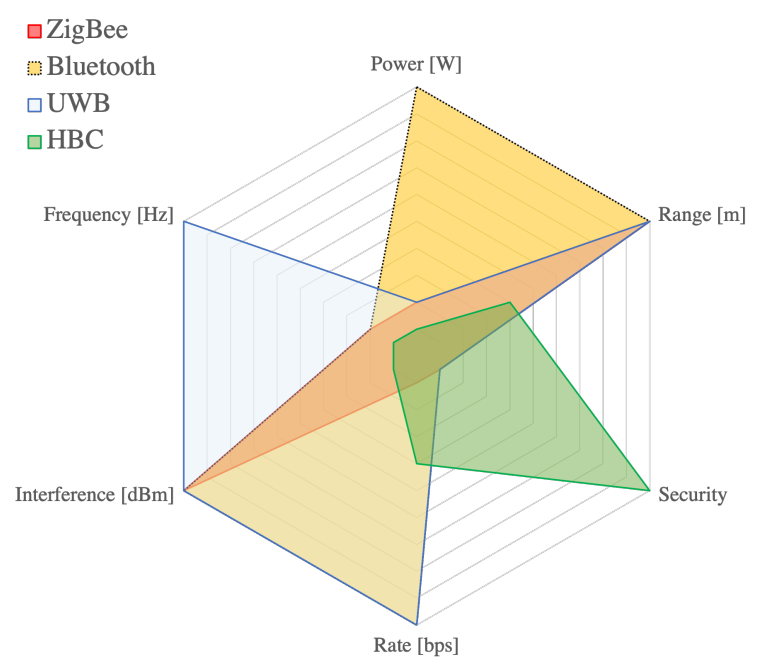

Fig. 4: A qualitative comparison of different WBAN technologies.

using simple modulation schemes of impulse radio (IRUWB) and frequency modulation (FM-UWB) with noncoherent detection or differentially coherent detection.

All PHY solutions share a single MAC protocol that has three modes of operation; 1) beacon mode with beacon periods (i.e., superframes), 2) non-Beacon mode with superframes, and 3) non-beacon mode without superframes. Superframes consists of three phases; 1) exclusive access phase for transmission of critical/emergency data, 2) random-access phase for nodes using CSMA/CA, and 3) contention access phase for nodes using slotted ALOHA access. The IEEE 802.15.6 also supports three levels of security; Level-0) Unsecured communications, Level-1) Authenticated but not encrypted communications, and Level-2) Authenticated and encrypted communications for confidentiality and privacy.

Other requirements of the IEEE 802.15.6 can be summarized as follows: Transmission range upper-bound and lower-bound are set as three meters for in-body and on-body communications, respectively. For $95 \%$ of the best-performing links, the packet error rate should be no more than $10 \%$ for a 250 octet payload. Nodes should be able to join and leave the network in less than 3 seconds. Less than $125 \mathrm{~ms}$ and $250 \mathrm{~ms}$ latency should be provided for medical and nonmedical applications, respectively. The minimum (maximum) transmission power must be no more (less) than $-10(0) \mathrm{dBm}^{3}$ Lastly, up to 10 nodes could co-exist in a $6 \mathrm{~m}^{3}$ space.

3) Bluetooth Low Energy: Bluetooth is a whole protocol stack with two modes of operation: basic rate (BR) and low energy (LE) [12]. The BR is the classic Bluetooth that can provide up to $3 \mathrm{Mbps}$ with optional enhanced data rate (EHR). LE is especially developed for low-power low-rate cheap devices powered by button cell batteries. Bluetooth operates on $2.45 \mathrm{GHz}$ ISM band which is split into $2 \mathrm{MHz}$ wide 40 sub-bands. Bluetooth can provide up to $1 \mathrm{Mbps}$ by using Gaussian frequency shift keying (GFSK) modulation. It

\footnotetext{
${ }^{3}$ This complies with FCC's SAR specification of $1.6 \mathrm{~W} / \mathrm{kg}$ in $1 \mathrm{~g}$ body tissue.
}

also has two security modes; the former ensures data integrity and encryption with or without authentication while the latter do not provide encryption. Authentication and encryption are implemented by AES with 128-bit key length and chaining message authentication codes, respectively. Bluetooth also support privacy by allowing devices to use random addresses over the time, which can be revealed only with a proper key.

\section{Summary and Insights}

Based on the broad range of IoB applications, this section first presented requirements for QoS, safety, power efficiency, form-factor, security, confidentiality, and privacy. As a result of the conflicting objectives, there are inextricably interwoven relations among these requirements. For example, provisioning a high QoS demand (e.g., URLLC) requires sophisticated signal processing techniques and advanced communication modules. Resulting computation and hardware complexity inherently conflict with power efficient and small form factor design. Likewise, security and privacy measures have a direct impact on overall system design and network architecture due to its limitations of the QoS performance. Therefore, future IoB standards should sufficiently address these main requirements.

In this regard, we presented three main BAN standards as an initial point for communication and networking standard of the IoB: IEEE 802.15.4, IEEE 802.15.6, and Bluetooth LE. The IEEE 802.15.4 standard (i.e., ZigBee) is developed for generic low-cost low-power WPANs operating on ISM bands. However, it has been recognized as insufficient to address specifics of body area communications and to support stringent requirements of BAN applications. Similarly, Bluetooth was developed as a whole protocol stack operating also on ISM bands. Considering the ever-increasing number of IoT devices, ZigBee and Bluetooth suffer from high interference, co-existence issues, security threats, and being limited to onbody communication links. Although communication modules of almost all communication devices are capable of operating on ISM bands, these standards do not offer a complete solution due to their deficiency and vulnerability in supporting the wide range of IoB applications and their requirements. Alternatively, IEEE 802.15.6 is dedicated to BANs with three different PHY options: NB (MICS, WMTS, ISM, MBAN), UWB, and HBC. To provide readers with a better insight into these communication bands, we present a qualitative comparison of different technologies in Fig. 4. These wide variety of spectrum ranges pave the way for a better system design tailored to the specific needs of IoB applications because communication channels in-on-and-around the human body have significantly distinctive behaviors at different spectrum bands. In what follows, we delve into reasons behind these behavioral changes based on the electromagnetic properties of the human body.

\section{Human Body: Bioelectromagnetics, Phantoms, AND PROPAGATION MECHANISMS}

Bioelectromagnetics (BEM) is the study of the interaction between electromagnetic (EM) fields and biological entities such as living cells, tissues, and organs. Therefore, it provides 


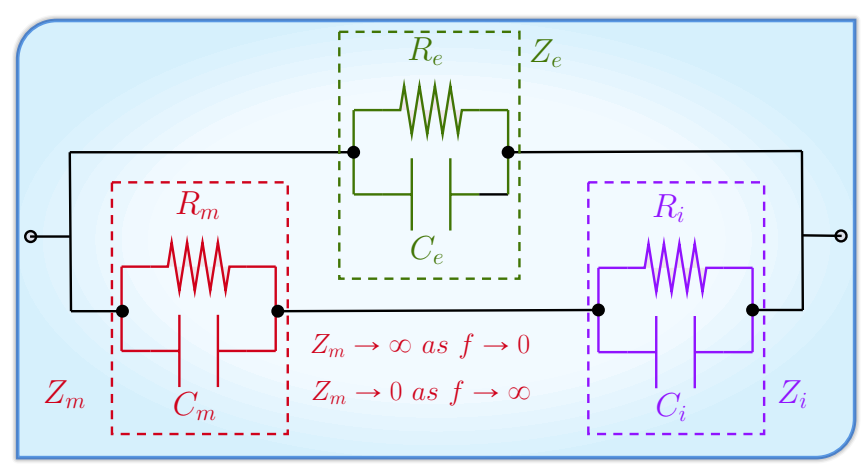

Fig. 5: Electric circuit representation of biological tissue.

the fundamental insights into the electromagnetic properties and behaviors of the human body. In this way, electromagnetic propagation characteristics in-on-and-around the human body can be understood and modeled in a precise manner. In this section, we first discuss dielectric properties of the human body, which are of utmost importance to understand the propagation characteristics and improve the accuracy of both analytical and numerical methods. Instead of human and animal experimentation, dielectric data can be useful to create body phantoms to mitigate cost, complexity, and ethical constraints. Second, we introduce a review of physical and numerical body phantoms. The third subsection presents EM computational tools that simulate EM propagation based on the numerical body phantoms.

\section{A. Bioelectromagnetics of Human Body}

The response of the human body to EM field exposures can be examined at the cellular and tissue level. The cellular level is characterized by dielectric properties of cell membranes as well as intracellular and extracellular fluids [62]; At only $10 \mathrm{~nm}$ of thickness, the cell membrane shows the highest resistance and capacitance as a result of its composition of phospholipids and proteins. On the other hand, the intracellular fluid has relatively higher resistance than the extracellular fluid that unites the cell to form the tissue. The electrical properties of the extracellular fluid are mainly determined by its moisture content and inorganic composition. Accordingly, the dielectric properties of tissue can be represented by an equivalent electric circuit [63], as shown in Fig. 5 where subscripts $m, i$, and $e$ refers to the cell membrane, the intracellular fluid, and the extracellular fluid, respectively. Notice in Fig. 5 that the cell membrane impedance $Z_{m}$ reaches zero and infinity as the frequency goes to zero and infinity, respectively. Accordingly, the equivalent electric circuit reduces to only $Z_{e}$ and a parallel circuit of $Z_{e} / Z_{i}$ at low and high frequencies, respectively. Since electrical properties of the human body varies with frequency, we next discuss the frequency-dependent dielectric properties including permittivity, conductivity, permeability, absorption, penetration depth, and diffraction.

1) Permittivity, Conductivity, and Permeability: Propagation mechanisms of EM fields are mainly characterized by three properties: permittivity is denoted by $\varepsilon$ and measured in $[\mathrm{F} / \mathrm{m}]$; conductivity is denoted by $\sigma$ measured in $[\mathrm{S} / \mathrm{m}]$;

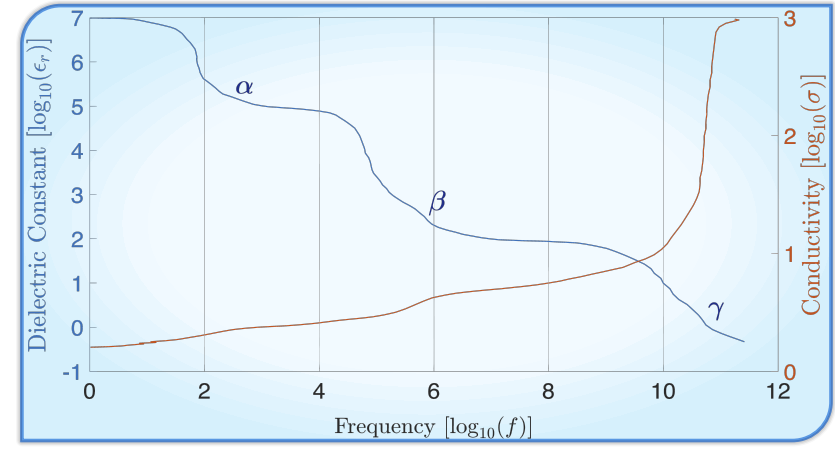

Fig. 6: Dielectric properties over the frequency spectrum; Permittivity and conductivity on the left and right y-axis, respectively.

and permeability is denoted by $\mu$ and measured in $[\mathrm{H} / \mathrm{m}]$ [61]. Since permeability of the nonmagnetic human body is considered to be almost the same with the free space [64], we focus our attention on frequency-dependent properties of permittivity and conductivity.

Permittivity is a complex property whose real and imaginary part are the dielectric constant $\varepsilon_{r}$ and loss factor, respectively. While the dielectric constant measures the polarity of medium, the loss factor is measure of the energy loss through conduction, polarization, and dissipation. On the other hand, conductivity results from loses caused by ionic conductivity and dielectric polarization [65]. Therefore, the human body is regarded as a frequency-dependent lossy dielectric medium. The permittivity is given by

$$
\varepsilon=\varepsilon_{0}\left(\epsilon_{r}-j \frac{\sigma}{\omega \varepsilon_{0}}\right)
$$

where $\varepsilon_{0}$ is the permittivity of free space, $\varepsilon_{r}$ is the relative permittivity (dielectric constant), $\omega=2 \pi f$, and $\sigma=\sigma_{i}+\sigma_{d}$ is the conductivity which is a superposition of ionic conductivity $\sigma_{i}$ and displacement conductivity $\sigma_{d}$. As shown in Fig. 6 , the frequency-dependent nature of the complex permittivity can be characterized by $\alpha, \beta$, and $\gamma$ dispersion regions corresponding to the frequency orders of $\mathrm{kHz}, \mathrm{MHz}$, and $\mathrm{GHz}$, respectively [66]. While the $\alpha$ dispersion is more related to ionic diffusion at the cell membrane, the $\beta$ dispersion is primarily due to the polarization of the cell membrane, which basically behaves as a barrier to ionic flows between intracellular and extracellular fluids. Lastly, $\gamma$ dispersion is constituted by the polarization effects of protein and other inorganic molecules.

The Debye's model is used as a basis to express the frequency-dependent nature of the dispersion, which is given by

$$
\varepsilon(\omega)=\varepsilon_{\infty}+\frac{\Delta \varepsilon}{1+j \omega \tau}
$$

where $\Delta \varepsilon=\varepsilon_{\infty}-\varepsilon_{s}$ is the dispersion magnitude, $\tau$ is the relaxation time constant of polarization, and $\varepsilon_{\infty}$ and $\varepsilon_{s}$ are the infinite $(\omega=\infty)$ and static $(\omega=0)$ permittivity, respectively. In order to account for different dispersion mechanics, Kenneth 
and Robert Cole extended the Debye's model to a N-Cole-Cole expression as follows [67]

$$
\varepsilon(\omega)=\frac{\sigma_{i}}{j \omega \varepsilon_{0}}+\sum_{i=1}^{N} \frac{\Delta \varepsilon}{1+\left(j \omega \tau_{n}\right)^{\alpha_{n}}}+\varepsilon_{\infty}
$$

where $\alpha_{n} \in[0,1]$ characterizes the deviation from Debye's behavior. Notice in (3) that the middle term represents $N$ different dispersion regions at intermediate frequencies whereas the first and last terms represent the dispersions at static and infinite frequencies, respectively. The early efforts on dielectric properties of different tissue types are presented in [68] where 20 different tissue types are measured over $1 \mathrm{MHz}$ to $20 \mathrm{GHz}$ spectrum range and key parameters are tabulated to compute above analytical models. An on-line database is available at FCC website ${ }^{4}$ which provides parameters of different tissue types to calculate 4-Cole-Cole expression for frequencies ranging from $10 \mathrm{MHz}$ to $6 \mathrm{GHz}$. These datasets show that high water content tissues (e.g., blood and muscle) have a higher permittivity and conductivity than the low-water content tissues (e.g., bone and fat). Since the water content of tissues reduces with age, aging has a negative impact on permittivity and conductivity [69].

2) Absorption and Penetration Depth: Another important frequency-dependent parameter is the number of wavelengths per unit distance, which is also referred to as the wavenumber, $k=k_{0} \sqrt{\mu \varepsilon}$ where $k_{0}$ is the free-space wavenumber and $\mu$ is the relative permeability. According to Beer-Lambert law, the intensity of the propagating electric field inside the human body is given by $E=E_{0} e^{j(\omega t-k d)}$ where $d$ is the propagation distance and $E_{0}$ is the electric field at the body-air surface. This clearly shows that the electric field exponentially decays with the product of wavenumber and distance, which differs from free space wave propagation. The penetration depth (skin) $\delta_{p}$ is also frequency-dependent as it is inversely proportional to the imaginary part of the wavenumber, i.e., $\delta_{p} \propto 1 / \operatorname{Im}(k)$. Since the wavenumber is a function of $\varepsilon$, the water content of tissue also has a direct impact on the penetration depth. That is, penetration depth increases with decreasing frequency and increasing water content. As a result, one can conclude that low frequencies are more suitable for in-body communications due to its high penetration depth and low propagation loss. On the other hand, higher frequencies yield a smaller SAR due to low penetration depth and high conductivity, which is obvious from $\mathrm{SAR}=\sigma E^{2} / \rho$ where $\rho$ is the mass density.

3) Diffraction: Diffraction is defined as the bending of waves around the corners of an obstacle or through an aperture into the region of geometrical shadow of the obstacle [70]. This phenomenon is observed when the wavelength is in the order of the obstacle size. Considering the uneven surface and non-uniform internal structure of the human body, diffraction is applicable for both in-body and on-body communications.

\section{B. Human Body Phantoms (HBPs)}

To validate the safety and evaluate the performance of IoB devices, their EM interaction with the human body must

\footnotetext{
${ }^{4}$ https://www.fcc.gov/general/body-tissue-dielectric-parameters
}

TABLE II: Comparison of the physical HBPs.

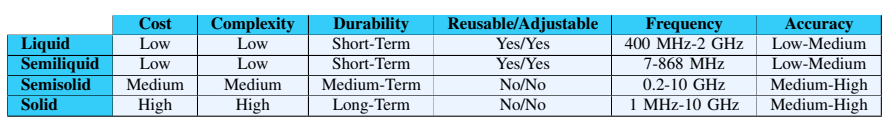

go through a quantitative and precise investigation in all the possible operation scenarios. However, regulatory bodies require experimental studies on human subjects and humanrelated materials to receive ethical clearance, which has strict constraints on scientific, physical, and psychological risk levels. To this end, body phantoms mimic dielectric properties of the human body by either numerical or physical models. Thus, body phantoms are useful to system modeling and optimization before human clinical trials. In addition to medical purposes (e.g., X-Ray, hyperthermia, MRI, diagnosis \& treatment), body phantoms are also used for SAR measurements, EM dosimetry, implantable \& wearable devices. The HBPs can be classified into physical and numerical for experimental and computational type of research, respectively. We provide a taxonomy of physical and numerical HBPs types in the sequel.

1) Physical HBPs: Based on the chemical compound ingredients, the physical HBPs are categorized into liquid HBPs, semiliquid HBPs, semisolid HBPs, and solid HBPs; which can be further categorized based on body parts (e.g., head, torso, limb, breast, etc.) or tissue types (e.g., low and high water content). Before delving into the details, we refer interested readers to Table $[\mathrm{II}$ for comparison among different physical HBPs.

a) Liquid HBPs: Liquid HBPs are the first and oldest phantom type that is especially suitable for high water content tissues with high dielectric properties. Therefore, water is the main ingredient whose permittivity and conductivity is adjusted to different tissue types by adding various solvable (e.g., sugar and salt) or dis-solvable (e.g., flour and oil) liquid mixtures. Fabrication procedure of several liquid phantoms is discussed in [110]. The prepared formulas are generally poured into thin containers with low dielectric properties. Depending on the application, the container may have a shape of regular prisms, body parts, or the whole body.

The liquid HBPs have the virtues of low-complexity and low-cost fabrication. It is also easy to place/replace the measurement probes and sensors. Liquid materials are reusable and adjustable to confine to different dielectric properties by adding extra ingredients. However, they have short-term durability because of the dehydration and mold growth [110]. Moreover, they are limited by frequency ranges up to $6 \mathrm{GHz}$ [111]. Due to the suspension insoluble ingredients, the formula is required to be stirred from time to time, which may result in minor changes in the electrical properties [112]. Lastly, the liquid HBPs may have inaccurate SAR measurements for two reasons [113]: 1) Dielectric properties of the container are different from that of liquid, and 2) The homogeneity of liquid formula also limits the accurate modeling of human body heterogeneity. In [114], [115], authors were the first to develop a multi-layer arm phantom for the five basic tissue layers: skin, fat, muscle, cortical bone, and bone marrow. The model's high accuracy and perfect match are validated by experiments on 
TABLE III: Categorization of physical HBPs based on body parts.

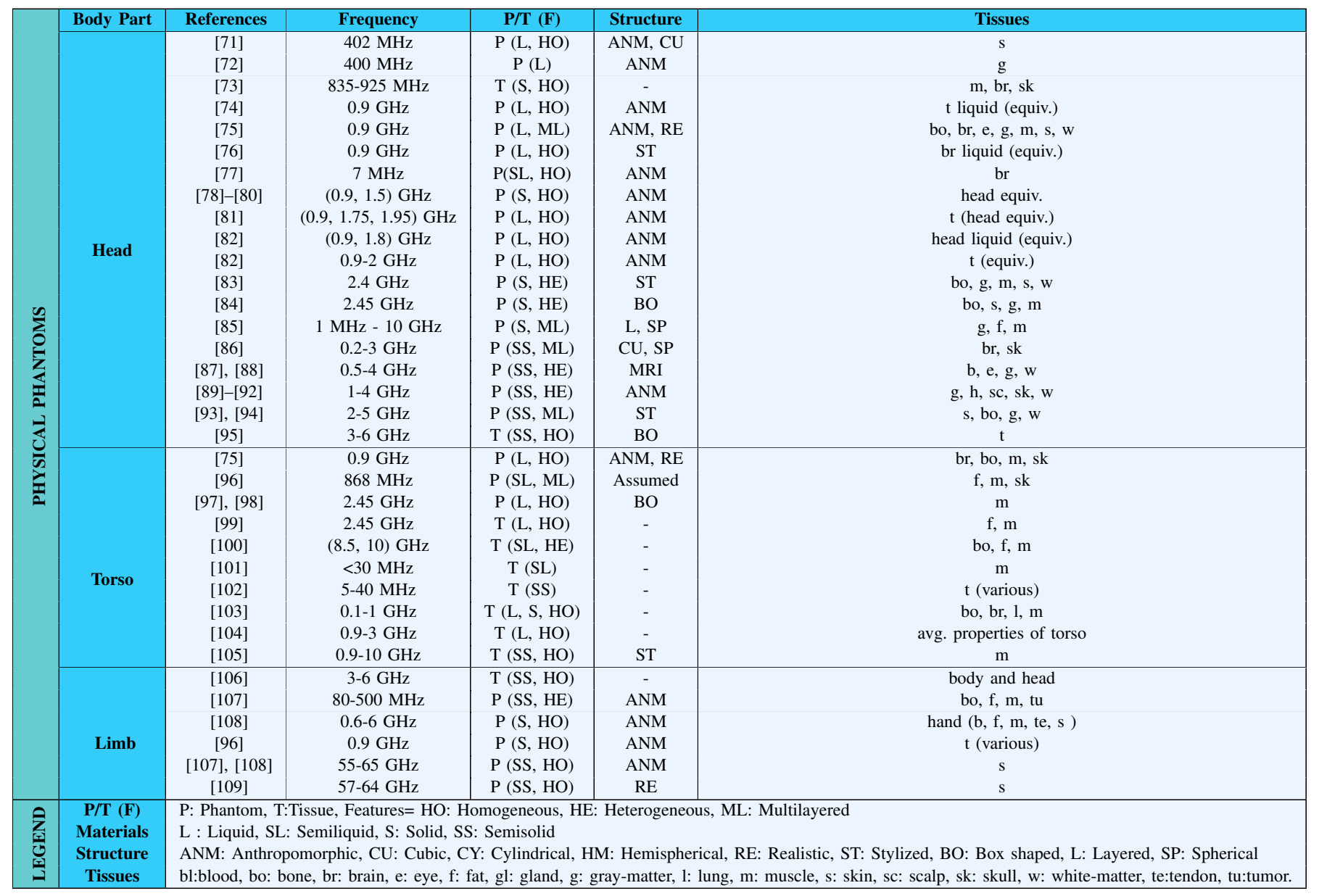

real subjects.

b) Semiliquid and Semisolid HBPs: Semiliquid (gel) and semisolid (jelly) phantoms are fabricated from coagulant materials such as gelatin, agar, polyamide resin, and polyethylene powder [116]. Unlike the liquid materials, the semiliquids do not suffer from suspension and sustain the homogeneity for a longer duration. Noting that the semiliquid share the rest of liquid phantoms' virtues and drawbacks, their fabrication takes a relatively long time as slow and continuous rotation is necessary to prevent trapping air bubbles [117].

Unlike the liquid and semiliquid HBPs, the semisolid materials can independently conform to any shape, which eliminates the need for an outer shell. Moreover, their nondiffusive and castable nature allows a multi-layered fabrication to mimic the anatomical structure of the human body more realistically. The fabrication procedure of semisolid materials is outlined in [118]. The semisolid HBPs have the virtues of medium-complexity and medium-cost fabrication. Even though the majority of the semisolid HBPs presented an accurate and stable nature for a wide frequency range up to 11 $\mathrm{GHz}$ [111], their accuracy is also studied at $55-65 \mathrm{GHz} \mathrm{mm}$ Wave bands [119], [120]. However, they have medium-term durability because of the dehydration and mold growth [118]. Moreover, replacing the measurement probes and sensors may cause the deformation of the phantom shape. Unlike the liquid and semiliquid HBPs, they are neither reusable nor adjustable.

c) Solid HBPs: Solid HBPs can be manufactured from a mixture of ceramic/graphite powder, silicone rubber, carbon fiber, and conductive plastics. Fabrication procedure of solid phantoms can be found in [121]. They can preserve their dielectric properties for an extended period of time since they are not subject to dehydration. However, their fabrication has high-complexity processes involving extreme pressures and temperatures. The complexity, as well as the required raw materials, increase the overall cost. Moreover, they are limited by frequency ranges up to $6 \mathrm{GHz}$ [111]. Similar to the semisolid HBPs, they are neither reusable nor adjustable, and it is not possible to replace the measurement probes and sensors. We refer interested readers to Table [III for a list of references categorized based on body parts.

2) Numerical HBPs: Thanks to the ever-increasing computational power, numerical simulations are powerful tools to analyze the radio propagation characteristics of the human body. Numerical HBPs are based on a digital representation of the human body anatomy, which is generally obtained by magnetic resonance imaging (MRI) and computed tomography (CT). MRI and CT provide gray-scale images of resolutions in the order of millimeters, which then goes through coloring and segmentation processes to interpret different colors into various tissue types. Virtual Population 3.0 [122], a family 


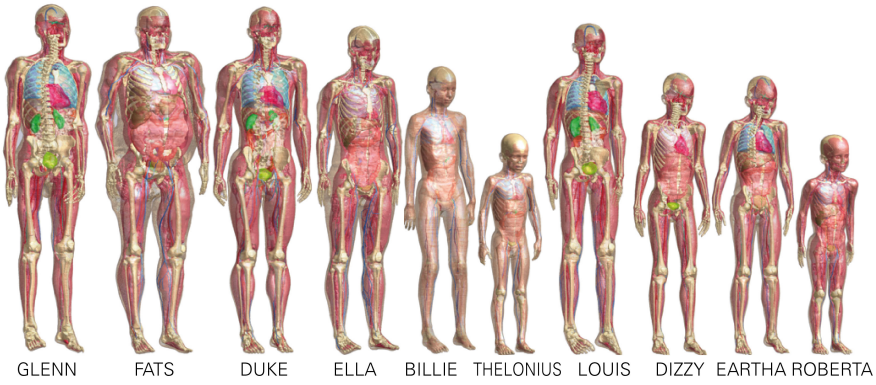

Fig. 7: Virtual Population 3.0 [122]: A family of numerical HBPs at different ages and genders.

of numerical HBPs at different ages and genders, are depicted in Fig. 7. In this way, each segment is associated with the corresponding permittivity and conductivity values to solve Maxwell equations [62]. Noting that segmentation is cumbersome and time-consuming, an alternative approach is using anthropomorphic numerical models, which are also referred to as dielectric analytical models. These models semiautomatically correlate the gray-scale images to the complex permittivity values by means of continuous transfer functions [123]. Numerical HBPs are categorized into homogeneous and heterogeneous models depending on the homogeneity of the tissue and phantom of interest. We refer interested readers to Table IV for a list of numerical HBPs discussed below.

a) Homogeneous HBPs: The homogeneous HBPs are generally in the form of simple geometric shapes such as cylinders, spheres, parallelepipeds, etc. They are generally used to evaluate EM dosimetry applications where the EM field is radiated from simple sources [113]. While spherical phantoms are mainly used for dosimetry inside the human head [124]-[127], cylindrical models are used for whole-body models [128], [129]. A 200- $\mathrm{mm}^{3}$ cube and a 200-mm diameter sphere models is also proposed in [130]. The Homogeneous HBPs are also used for the confirmation of the validity of numerical tools covered in the next sub-section.

b) Heterogeneous HBPs: The heterogeneous HBPs are also known as volumetric or voxel phantoms as they consist of volumetric cells, i.e., voxels. Their accuracy increases with the number of voxels at the expense of more computation and time complexity. Since the human body exhibits different dielectric properties of different ages and genders, some models provide a set of male and female HBPs at various ages, such as Virtual Family [131], Virtual Population 3.0 [122], and GSF family [132]. There are also phantoms dedicated to different nations such as the Visible Human Model of U.S. National Library of Medicine [133], Japanese male and female model [134], and Chinese adult model [135].

\section{Numerical Propagation Characterization Methods}

Apart from dosimetry applications and SAR measurements, numerical modeling of RF propagation has distinct features since the human body acts as a transmission medium and/or reflectors. If transceivers are located in-on-and-around the human body, radio propagation paths and their gains undergo large variations, especially when the polarization and orientation of antennas change with the body postures. Such variations are significant for microwaves because body dimensions are large compared to the wavelength. Thus, semianalytical computational EM approaches are extremely useful to investigate the radio propagation around the human body and gain a deep insight into the underlying physics. On the contrary of simplified theoretical model and analyses, numerical simulations can afford the simulation of very realistic and accurate scenarios based on the numerical phantoms presented in the previous section. In the following sections, we briefly outline wide-spread computational EM methods.

1) Ray Tracing $(R T)$ : Scattering, reflection, and penetration are the three basic propagation phenomena to describe the interaction between the surrounding environment and the propagating waves. If the objects are larger than multiple signal wavelengths, RT techniques can provide efficient and accurate results based on high-frequency asymptotic methods. Based on geometrical optics, the geometrical theory of diffraction (GTD) can calculate the reflected and refracted fields from and through surfaces [70]. Since it ignores the diffractions from curved surfaces and corners, GTD is inefficient in taking the multiple reflections into account, which can be mitigated by the uniform theory of diffraction (UTD) [136]. RT is highly popular, especially with the accurate and efficient calculation of key parameters such as power delay profile, received signal strength, delay spread, and angle of arrival [113]. Therefore, RT techniques are also studied in bodycentric communications [136]-[141].

2) Method of Moment $(M o M)$ : This method is developed to solve complex integrals by reducing them to a system of simpler linear equations using Harrington's weighted residuals techniques [142]. It is especially efficient for cases where volume is large compared to the surface such that solving Maxwell's equations over the entire volume of interest is not necessary. The MoM has a time complexity of $\mathcal{O}\left(N^{2}\right)$ and is not suitable for parallel computing methods [111], which limits its use to simple homogeneous geometries. Therefore, complexity poses considerable restrain on its application to IoB, where the received signal strength in/on the human body is required to be known. The MoM is mostly suitable for thin-wire structures and applicable in both time and frequency domains. Therefore, it is generally employed to study body propagation of loop wire antennas [143]-[147].

3) Finite Element Method (FEM): FEM analyses EM structures by dividing them into a number of isoparametric elements in various shapes, e.g., rectangular and triangular. In this way, it is suitable for the propagation analysis of structures with curved boundaries. Since it is formulated by a set of linear equations, a major disadvantage of FEM is the time complexity of matrix solution. Denoting the number of rows and columns of stiffness matrix by the $N$ and $W$, Farmaga et al. evaluated the complexity order of FEM as $\mathcal{O}\left(N W^{2}\right)$ [148], which turns out to be the highest among other numerical approaches. Since it is well-suited to $\mathrm{MHz}$ frequencies, it is mostly used for IoB applications operating at HBC frequencies [149]-[154] [c.f. Section V-B2].

4) Finite-Difference Time-Domain (FDTD): Since it was proposed by Yee in 1966 [155], FDTD has become one of 
TABLE IV: Numerical Body Phantoms

\begin{tabular}{|c|c|c|c|c|c|}
\hline Type & References & Model/Shape & Gender & Age & Resolution (mm) \\
\hline 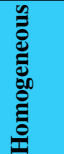 & $\begin{array}{c}[71], 75], 79] \\
124] \\
125]-127 \\
128 \\
129 \\
12]\end{array}$ & $\begin{array}{c}\text { Cylindrical Arm Model } \\
\text { Spherical phantom } \\
\text { Multilayered sphere model of the human skull } \\
\text { Cylindrical model for whole body } \\
\text { Three-layered elliptical model of the human body }\end{array}$ & $\begin{array}{l}- \\
- \\
- \\
- \\
-\end{array}$ & $\begin{array}{c}0-90 \\
- \\
- \\
- \\
-\end{array}$ & $\begin{array}{l}- \\
- \\
- \\
- \\
-\end{array}$ \\
\hline 竎 & \begin{tabular}{|c|}
$122],[131]$ \\
$123]$ \\
$132]$ \\
133 \\
134 \\
135 \\
\end{tabular} & $\begin{array}{c}\text { Virtual population } \\
\text { Virtual Family } \\
\text { Dielectric anatomical model } \\
\text { GSF family } \\
\text { Visible human model } \\
\text { Japanese Avg. Male and Female } \\
\text { Chinese Visible Human project }\end{array}$ & $\begin{array}{c}\text { All } \\
\text { F, A } \\
\text { F, M, A } \\
\text { M, F, C } \\
\text { M, A } \\
\text { F, M, A } \\
\text { C } \\
- \\
\text { F, M, A } \\
\text { F, M, A }\end{array}$ & $\begin{array}{c}5-84 \\
26 \\
26-34 \\
6-11 \\
34 \\
38-40 \\
2,7 \\
- \\
22 \\
22-35\end{array}$ & $\begin{array}{l}\text { Head: } 0.5 \times 0.5 \times 1.0 \\
\text { Torso + Limb: } 0.9 \times 0.9 \times 2.0 \\
\text { Body: } 2 \\
\text { Implant: } 0.5 \\
\text { Head: } 0.5 \times 0.5 \times 1.0 \\
\text { Torso + Limb: } 0.9 \times 0.9 \times 2.0 \\
2 \times 2 \times 2 \\
- \\
- \\
- \\
- \\
2 \times 2 \times 2 \\
1 \times 1 \times 1\end{array}$ \\
\hline
\end{tabular}

the best-known and widely-adopted numerical methods in computational electromagnetics. Similar to FEM, it is capable of analyzing large, complex, and heterogeneous EM structures by dividing them into simpler elements. Unlike the high complexity of the previous methods, FDTD has a linear time complexity $\mathcal{O}(N)$ and suitable for parallel computing methods to handle large EM structures in finer resolutions [156]. However, this low complexity is at the expense of not being as flexible as the FEM method on curvy EM structures such as the human body [113]. The FDTD has found its place to model human head or entire body for SAR measurement purposes [157]-[161]. This method is also used in HBC application to measure the electric field around the human body [162]-[166]. It is shown in [164], [165] that most of the electric field is concentrated around the tip and surface of the arm [c.f. Section V-B2.

\section{Summary and Insights}

This section provided the fundamental insights into the electromagnetic properties (e.g., permittivity, conductivity, permeability) and behaviors (e.g., absorption, penetration depth, and diffraction) of the human body. The tools and models presented help researchers gain a deeper understanding and perform accurate modeling of the propagation characteristics in-on-and-around the human body. Since dielectric properties are frequency dependent, overall propagation loss of in/on body links heavily depends on frequency, distance, and tissue properties along the propagation paths. Unlike the in-body links, the communication on and around the human body is jointly determined with electromagnetic interaction with the surrounding environment.

In order to validate the safety and characterize the channels, it is necessary to investigate EM interaction of IoB devices in a quantitative and precise manner. However, ethical constraints on conducting experiments on living subjects has led to development of physical and numerical body phantoms that mimic the dielectric properties of the human body. Accordingly, we surveyed physical (e.g, liquid, semi-liquid, semi-solid, solid) and numerical (e.g., homogeneous and heterogeneous) phantoms in a comprehensive manner. Indeed, body phantoms are extensively used for SAR measurements and channel characterization especially for in/on body communication devices due to the technical challenges in locating transceivers inside the body [c.f. Section IV]. The numerical body phantoms are particularly important for developing numerical propagation characterization methods such as RT, MoM, FEM, and FDTD. FEM and FDTD have been mostly used to validate the analytical and empirical HBC channel models [c.f. Section V].

\section{NB \& UWB Channel Modeling}

An accurate channel model is an initial and essential step for well-designed IoB communication and networking systems. However, modeling the communication channels within the proximity of the human body substantially differs from traditional communications. This is mainly because of the heterogeneous frequency-dependent dielectric nature of the human body that has relatively high permittivity and conductivity. Therefore, signal attenuation is mainly affected by the carrier frequency, the distance between transceivers as well as tissue dielectric properties along the propagation path, body curvatures, changing body postures, and so on.

For the sake of a better organization, we define three different IoB types based on their location on the human body:

I: In-Body IoB nodes are either implanted or embedded devices located in deep tissues/organs or under the human skin, respectively;

O: On-Body IoB nodes are located on or within 2 centimeters around the body surface;

E: Off-Body IoB nodes are external devices located 2 centimeters to several meters away from the body surface;

which results in the following combination of link types

I/I: In-Body to In-Body

I/O: In-Body to On-Body

I/E: In-Body to Off-Body

O/O: On-Body to On-Body

O/E: On-Body to Off-Body 
Depending on the IoB node location, the link budgets may be dominated by various channel characteristics. For example, inbody links (I/I and I/O) suffer from severe signal attenuation due to absorption and scattering effects of heterogeneous body tissues, whereas multipath fading and shadowing are more significant for on-body links $(\mathrm{O} / \mathrm{O}$ and $\mathrm{O} / \mathrm{E})$ due to body postures, body movements, and reflecting surfaces in the surrounding environment. Of course, these links exhibit distinct behaviors at different node locations and frequency bands.

In the remainder of this section, we investigate NB \& UWB channel modeling based on the first-order and the second-order channel statistics. While the first-order statistics account for mean and covariance of the signal attenuation, the secondorder statistics are related to a variety of communication paradigms such as delay spread, power delay profile, levelcrossing rate, Doppler spread, auto-correlation and crosscorrelation, and so on.

\section{A. The First-Order Channel Statistics (FOCS)}

Based on the magnitude of variation in signal decay, the FOCS can be categorized into large-scale and small-scale signal attenuation. Hence, path loss and shadowing contribute to the large-scale FOCS, while multipath fading is the main reason for slight variations in the signal attenuation.

1) Large-Scale FOCS (Path Loss \& Shadowing): The large scale signal attenuation is mainly characterized by two phenomena; absorption and scattering. While the former is due to the lossy dielectric properties of body tissues, the latter is because of the heterogeneity of body tissues. On the other hand, shadowing is defined as random variations in signal decay and caused by creeping waves at low frequencies and diffractions existing in shadowed regions of the body surface. Hence, large-scale fading is often presented as a combination of path loss and shadowing, which are often represented by five different parametric models:

The first and the most basic model assumes that path loss $\mathrm{PL}(\mathrm{d})[\mathrm{dB}]$ linearly varies with distance $\mathrm{d}$

Model A: $\mathrm{PL}(\mathrm{d})=\mathrm{C}+\alpha \mathrm{d}+\mathrm{S}$

where $\mathrm{d}[\mathrm{m}]$ is the distance between IoB nodes, $\mathrm{C}[\mathrm{dB}]$ is a constant term that generally refers to the path loss at a reference distance, $\alpha$ is the slope parameter, and $\mathrm{S}[\mathrm{dB}]$ is the log-normal shadowing component that follows a Normal distribution with a standard deviation of $\sigma_{s}, \mathrm{~S} \sim \mathcal{N}\left(0, \sigma_{\mathrm{s}}^{2}\right)$. In the second model, path loss varies with a power of the distance as follows

Model B: $\mathrm{PL}(\mathrm{d})=\mathrm{C}+\alpha \mathrm{d}^{\mathrm{n}}+\mathrm{S}$

where $\mathrm{n}$ is the path loss exponent. The third model combines log-distance path loss model with log-normal shadowing for a given reference distance

Model C: $\mathrm{PL}(\mathrm{d})=\mathrm{PL}\left(\mathrm{d}_{0}\right)+10 \mathrm{n} \log _{10}\left(\frac{\mathrm{d}}{\mathrm{d}_{0}}\right)+\mathrm{S}$,

where $\operatorname{PL}\left(\mathrm{d}_{0}\right)[\mathrm{dB}]$ is the path loss at the reference distance $\mathrm{d}_{0}[\mathrm{~m}]$. Notice that (6) reduces to free-space radio propagation
TABLE V: In-Body Parametric Path Loss Models

\begin{tabular}{|c|c|c|c|c|c|}
\hline \multicolumn{6}{|c|}{ In-Body Parametric Path Loss Models } \\
\hline Ref. & Band & Frequency & Model & Link & Description \\
\hline 167] & MICS & $402-405 \mathrm{MHz}$ & Model C & $\begin{array}{c}\mathrm{I} / \mathrm{I} \\
\mathrm{I} / \mathrm{O}\end{array}$ & IEEE Std. 802.15.6 \\
\hline 168] & $\begin{array}{c}\text { MICS } \\
\text { WMTS } \\
\text { ISM }\end{array}$ & $\begin{array}{c}402-405 \mathrm{MHz} \\
863-870 \mathrm{MHz} \\
2.4-2.5 \mathrm{GHz}\end{array}$ & Model C & $\begin{array}{l}\mathrm{I} / \mathrm{I} \\
\mathrm{I} / \mathrm{O}\end{array}$ & $\begin{array}{l}\text { SIM: Finite integration technique } \\
\text { EXP: Phantom Body }\end{array}$ \\
\hline 169] & $\begin{array}{l}\text { MICS } \\
\text { ISM }\end{array}$ & $\begin{array}{l}402-405 \mathrm{MHz} \\
2.4-2.5 \mathrm{GHz}\end{array}$ & Model D & $\mathrm{I} / \mathrm{O}$ & EXP: Human/Phantom Body \\
\hline 170] & ISM & $2.4-2.5 \mathrm{GHz}$ & $\begin{array}{l}\text { Model A* } \\
\text { Model C* }\end{array}$ & $\begin{array}{l}\text { I/I } \\
\mathrm{I} / \mathrm{O} \\
\mathrm{I} / \mathrm{E}\end{array}$ & STAT: Liquid phantom \\
\hline [171] & UWB & $1-6 \mathrm{GHz}$ & Model B & $\mathrm{I} / \mathrm{I}$ & SIM: Finite integration technique \\
\hline 172] & UWB & $3.5-4.5 \mathrm{GHz}$ & Model B & $\mathrm{I} / \mathrm{I}$ & $\begin{array}{l}\text { SIM: CST Studio software } \\
\text { EXP: Pig tissues }\end{array}$ \\
\hline 173] & UWB & $3.4-4.8 . \mathrm{GHz}$ & Model C* & $\mathrm{I} / \mathrm{O}$ & SIM: FDTD, EXP: Liquid Phantom \\
\hline [174] & UWB & $3.4-4.8 . \mathrm{GHz}$ & Model C & $\mathrm{I} / \mathrm{O}$ & SIM: FDTD \\
\hline 175] & UWB & $2-6 \mathrm{GHz}$ & Model C & $\mathrm{I} / \mathrm{I}$ & $\begin{array}{l}\text { SIM: Frequency dependent path } \\
\text { loss based on FDTD simulations. }\end{array}$ \\
\hline
\end{tabular}

model for $\mathrm{n}=2$. The fourth model employs the following linear fitting model

Model D: $\mathrm{PL}(\mathrm{d})=\alpha \log _{10}(\mathrm{~d})+\beta+\mathrm{S}$,

where $\alpha$ and $\beta$ are linear fitting coefficients. The last model follows an exponential decay that flattens out for large distance as a result of the contribution of multipath components from indoor environment

Model E: $\mathrm{PL}(\mathrm{d})=-10 \log _{10}\left(\mathrm{P}_{0} \mathrm{e}^{-\mathrm{M}_{0} \mathrm{~d}}+\mathrm{P}_{1} \mathrm{e}^{-\mathrm{M}_{1} \mathrm{~d}}\right)+\mathrm{S}$,

where $\mathrm{P}_{0}[\mathrm{~dB}]$ is the average loss close to the antenna; $\mathrm{P}_{1}$ $[\mathrm{dB}]$ is the average attenuation of the transmitted signal that is reflected back by indoor environment; $M_{0}[\mathrm{~dB} / \mathrm{cm}]$ is the average decay rate for the surface wave traveling around the perimeter of the body; and $\mathrm{M}_{1}[\mathrm{~dB} / \mathrm{cm}]$ is the average decay rate for reflected signals.

2) In-Body Link Budgets (I/I-I/O-I/E): The implanted IoB devices support a wide variety of medical applications such as defibrillators, cardiac pacemakers, swallowable endoscopy capsules, glucose and bladder pressure monitors, smart pills for precise drug delivery to a target body location, and micro-robots used for the execution of biopsy and therapeutic procedures [176]. Due to ethical concerns and technical challenges, experimental in-body channel measurements are not possible on human subjects. Therefore, the implant channel characterization campaigns are carried out through measurements on experimental body phantoms or through simulations on numerical body phantoms, as explained in the previous section. There are two potential spectrum bands for in-body communication: MICS and lower UWB.

IEEE 802.15.6 standard allocated the MICS band for in-body links thanks to its favorable propagation behavior through human tissues and support for the use of smallsize antennas. The Channel Modeling Subgroup of TG15.6 determined that Model $\mathrm{C}$ in (6) is a good fit for in-body channels. The mean value of in-body signal attenuation is caused by the lossy dielectric nature of human tissues whilst shadowing is because of the varying dielectric properties of different organs/tissues along the propagation path [33]. In order to obtain a statistical channel model, a 3D immersive visualization and simulation platform is developed, and measurements are obtained for near-surface (cardiac pacemakers) 


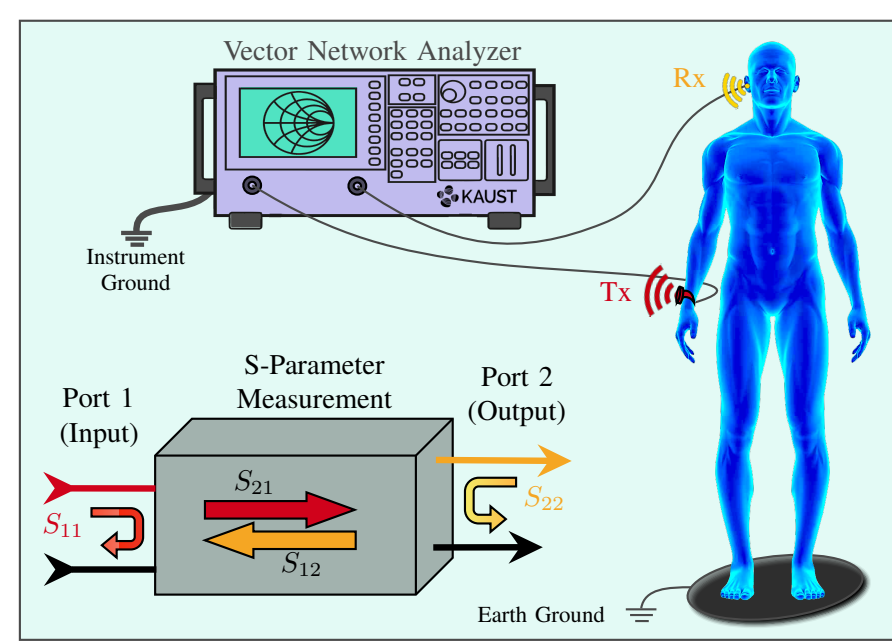

Fig. 8: Illustration of measuring S-parameters between a smart watch and wireless headphone.

and deep tissue (endoscopy capsule) devices [167], [177], [178].

Albeit having favorable in-body propagation characteristics, the limited bandwidth of MICS systems does not support highspeed communication. Hence, UWB systems have emerged as an attractive alternative with the following attributes [179]: As a result of the low maximum effective isotropic radiated power (EIRP) spectral density, noise-like nature of UWB signals makes signal detection hard to unintended receivers. This inherent UWB feature increases the robustness of UWB systems against jamming, thereby mitigating the need for sophisticated encryption algorithms in low-cost transceivers [33]. Moreover, the simple structure impulse radio (IR) UWB transceivers facilitate miniature and low-power UWB systems. Table $\mathrm{V}$ lists different frequency bands and parametric path loss models used for $\mathrm{I} / \mathrm{I}$ and $\mathrm{I} / \mathrm{O}$ link configurations.

3) On-Body Link Budgets (O/O-O/E): Unlike the regular outdoor and indoor RF channel characterization, propagation between two points on the human body has subtle distinctions. Although off-body communication (e.g., from an access point to a mobile handset) considers a sitting/standing person stationary, this is not a valid assumption for channel characterization between transceivers located on the human body. In addition to the environmental changes, the on-body link budgets also substantially differ from its off-body counterpart in being affected by the changes in body postures and gaits. For instance, the on-body link of a sitting person could be lineof-sight (LoS) or non-line-of-sight (NLoS) based on the body posture, which is generally considered the same in off-body channel characterization.

The parametric path loss models for the on-body links are tabulated in Table VI .Since technical and ethical restrictions of in-body communication do not apply for on-body communications, the majority of works consider statistical channel modeling. The experiments are often conducted in an indoor environment by measuring S-parameters using a fixed or hand-held vector network analyzer if the human subject is stationary or mobile, respectively. The illustration
TABLE VI: On-Body Parametric Path Loss Models

\begin{tabular}{|c|c|c|c|c|c|}
\hline \multicolumn{6}{|c|}{ On-Body Parametric Path Loss Models } \\
\hline Ref. & Band & Frequency & Model & Link & Description \\
\hline [180] & $\begin{array}{l}\text { MICS } \\
\text { WMTS } \\
\text { ISM } \\
\text { UWB }\end{array}$ & $\begin{array}{c}400-450 \mathrm{MHz} \\
608-614 \mathrm{MHz} \\
950-956 \mathrm{MHz} \\
2.4-2.5 \mathrm{GHz} \\
3.1-10.6 \mathrm{GHz}\end{array}$ & Model D & $\mathrm{O} / \mathrm{O}$ & $\begin{array}{l}\text { IEEE Std. } 802.15 .6 \\
\text { STAT: Body phantom meas. } \\
\text { in hospital room and } \\
\text { anechoic chamber. }\end{array}$ \\
\hline [181] & $\begin{array}{l}\text { ISM } \\
\text { UWB }\end{array}$ & $\begin{array}{c}902-928 \mathrm{MHz} \\
2.4-2.5 \mathrm{GHz} \\
3.1-10.6 \mathrm{GHz}\end{array}$ & $\begin{array}{l}\text { Model E } \\
\text { Model C }\end{array}$ & $\begin{array}{l}\mathrm{O} / \mathrm{O} \\
\mathrm{O} / \mathrm{O}\end{array}$ & $\begin{array}{l}\text { IEEE Std. } 802.15 .6 \\
\text { STAT: Body phantom meas. }\end{array}$ \\
\hline 182 & ISM & $2.4-2.5 \mathrm{GHz}$ & Model C & $\mathrm{O} / \mathrm{O}$ & $\begin{array}{l}\text { STAT: Human body meas. } \\
\text { SIM: FDTD }\end{array}$ \\
\hline [183] & ISM & $\begin{array}{c}902-928 \mathrm{MHz} \\
2.4-2.5 \mathrm{GHz}\end{array}$ & $\begin{array}{l}\text { Model C } \\
\text { Model E }\end{array}$ & $\mathrm{O} / \mathrm{O}$ & STAT: Indoor human body meas. \\
\hline$[184$ & UWB & $3-6 \mathrm{GHz}$ & Model C & $\mathrm{O} / \mathrm{O}$ & STAT: Indoor human body meas. \\
\hline$[185$ & UWB & 3-6 GHz & Model C & $\mathrm{O} / \mathrm{O}$ & STAT: Indoor human body meas. \\
\hline$\overline{186}$ & UWB & $4.2 \mathrm{GHz}$ & Model C & $\mathrm{O} / \mathrm{O}$ & STAT: Indoor human body meas. \\
\hline [187] & UWB & $3-10 \mathrm{GHz}$ & Model C & $\mathrm{O} / \mathrm{O}$ & STAT: Indoor human body meas. \\
\hline 188 & UWB & $2-8 \mathrm{GHz}$ & Model C & $\mathrm{O} / \mathrm{O}$ & STAT: Indoor human body meas. \\
\hline [189] & UWB & $3-6 \mathrm{GHz}$ & Model C & $\mathrm{O} / \mathrm{O}$ & STAT: Indoor human body meas. \\
\hline$\overline{190}$ & UWB & $3-10 \mathrm{GHz}$ & Model C & $\mathrm{O} / \mathrm{O}$ & STAT: Indoor human body meas. \\
\hline 191] & UWB & 3-6 GHz & Model C & $\mathrm{O} / \mathrm{O}$ & STAT: Indoor human body meas. \\
\hline$\overline{192}$ & UWB & $3-9 \mathrm{GHz}$ & Model D & $\mathrm{O} / \mathrm{O}$ & STAT: Liquid phantom meas. \\
\hline [193] & ISM & $2.4-2.5 \mathrm{GHz}$ & Model C & $\mathrm{O} / \mathrm{E}$ & $\begin{array}{l}\text { STAT: Indoor human body meas. } \\
\text { with various body postures. }\end{array}$ \\
\hline$\overline{194]}$ & UWB & 3-10 GHz & Model C & $\mathrm{O} / \mathrm{O}$ & SIM: Distance and freq. depend. \\
\hline [195] & ISM & $2.4-2.5 \mathrm{GHz}$ & Model C & $\mathrm{O} / \mathrm{E}$ & $\begin{array}{l}\text { STAT: Indoor human body meas. } \\
\text { with various body postures. }\end{array}$ \\
\hline [196] & UWB & $2-10 \mathrm{GHz}$ & Model C & $\mathrm{O} / \mathrm{E}$ & $\begin{array}{l}\text { STAT: Indoor human body meas. } \\
\text { with various body postures. }\end{array}$ \\
\hline
\end{tabular}

of S-parameter measurement can be seen in Fig. 8 . From all these statistical channel campaigns, one can infer that the following three critical factors have a significant impact on the measurements:

- Although the parametric models capture the distancedependent behavioral pattern, reported measurements highly depend on experimental setup and environment. Even measurement setups sharing the same parametric model within similar environments report a wide range of parameter values depending on the operational frequency and transceiver locations. For example, belt-to-chest, beltto-head, belt-to-wrist links were respectively reported to have $38.9 \mathrm{~dB}, 41 \mathrm{~dB}$, and $46.3 \mathrm{~dB}$ of average path-loss, which is proportional to the distance [29]. Notice that link fluctuations due to the changes in body postures and gaits become more significant during walking, running, and sports activities. For example, the peak-to-peak variation of the belt-to-chest link was recorded to be $8 \mathrm{~dB}$ and 21 $\mathrm{dB}$ during standing and moving scenarios, respectively. These variations were much higher for chest-to-head links, which were $44 \mathrm{~dB}$ and $56 \mathrm{~dB}$ in the stationary and mobile scenarios, respectively. It is even possible to observe around $4 \mathrm{~dB}$ variations due to involuntary body movements (e.g., breathing) [197]. Therefore, the generalization of these parametric models is not possible without a large parameter data set tailored to node locations and operational frequency.

- Some of the works have recorded significant differences between measurements conducted within an anechoic chamber and daily-life indoor environments. This makes clear that multipath fading has an impact as noticeable as the large-scale fading. Therefore, anechoic chambers are necessary to strip the multipath fading component away and observe the large scale fading alone. 
- Channel measurements are typically done by allowing transceivers to share the instrument ground of vector network analyzer (VNA) [c.f. Fig. 8]. This is hardly the case for wearable devices in practice and ignores the coupling effects. Although de-embedding antenna characteristics from propagation paths are generally neglected for on-body links, its momentous impacts on human body communication channel characterization are thoroughly investigated [c.f. Section V].

4) Small-Scale FOCS (Multipath Fading): As a result of body motions and posture changes, multiple paths are formed between the transmitter and receiver. Consequently, a received signal may end up as the superimposition of several delayed, attenuated, time-varying, and eventually distorted replicas of a transmitted signal [62]. The first-order small-scale statistical modeling studies mostly focus on on-body links as they are most vulnerable to multipath fading phenomena. Measured or simulated channel gain data is generally fitted to statistical distributions, which are commonly used to describe fading effects. These common small-scale fading distributions can be presented under the umbrella of generalized gamma distribution as follow

$$
f(x \mid a, b, c)=\frac{p x^{a p-1}}{b^{a p} \Gamma(a)} \exp \left[-\left(\frac{x}{b}\right)^{p}\right]
$$

where $\Gamma(\cdot)$ is the standard Gamma function, $a$ and $p$ are shape parameters, and $b$ is the scale parameter. The generalized gamma distribution can reduce to Rayleigh distribution ( $p=2, a=1)$, Nakagami- $m$ distribution $(p=2)$, Weibull distribution $(a=1)$, and gamma distribution $(p=1)$, and log-normal distribution $\left(p \rightarrow 0, a=2 /\left(p^{2} b^{p}\right)\right.$ ). Based on more than 200 statistical fits, a comprehensive comparison of these distributions are provided in [34, \& references therein]. Log-normal, Weibull, and gamma have been mostly studied and found often to be best fits. In particular, gamma/Weibull and log-normal/gamma distributions fit better to everyday and dynamic activities, respectively.

Although Nakagami-m, Rician, and Rayleigh distributions are commonly preferred to characterize small-scale fading in regular RF channels, they have been shown to provide poor fits for the on-body measurements [34]. This is again due to the aforementioned distinctions of communication on-and-around the human body. For NB channels, there is a general trend toward modeling small-scale fading by Weibull and gamma distribution [198].

Since the channel gain is a product of numerous factors (e.g., diffraction, reflection, absorption, antenna gain, etc.), their contribution to the signal attenuation is additive in the log-domain. Since the addition of multiple log-normally distributed paths still yields another log-normal distribution, log-normal distribution performs well to capture these multiplicative effects in additive $\mathrm{dB}$ scale. This is especially true for UWB channels with larger bandwidths. Therefore, in comparison to NB channels, large-scale fading component is much higher for UWB channels due to higher frequencies [167]. This can also be observed for shadowing as shown in Table $\mathrm{V}$ and Table $\mathrm{VI}$.
In light of the above discussions, one can infer that smallscale fading statistics are also subject to node locations, dynamicity of the human body, carrier frequency, bandwidth, and surrounding environment. In order to decide on which statistical distribution is the best option for a specific scenario, potential distributions should be compared based on a 'goodness-of-fit' criterion, which typically measures the discrepancy between observed values and the expected values under the target model. For instance, Akaike and Bayesian information criteria are two well-known tools that strike a balance between the fitness and simplicity of a model.

\section{B. The Second-Order Channel Statistics (SOCS)}

FOCS concentrate on time-invariant components of the wireless channel and target mean/variance of the underlying distribution model. However, they are not sufficient to characterize the time-variant nature of channels. The propagation path on a walking/running human body randomly changes with time since the number of reflectors and their locations within the environment changes. This inevitably causes random changes in delays, amplitudes, and multipath components. At this point, it is crucial to understand timedependent variations of on-body links.

1) Delay spread and Power Delay Profile: In multipath channels, the time delay spread is an important metric defined as the time between the first and the last received signal component of a transmitted signal. The delay spread is random especially for mobile terminals. For example, the propagation path of a left-wrist-to-right-hip link on a moving human subject randomly changes not only with the changing environment but also due to the swinging arms which expose and block lineof-sight link. If the delay spread is smaller than the inverse of the bandwidth, then all multipath components (LoS and NLoS) are unresolvable, which is typically the case for NB signals. In [183], authors investigate the root mean square (RMS) delay spread for $915 \mathrm{MHz}$ and $2.45 \mathrm{GHz}$ with $15 \mathrm{~cm}$ and $45 \mathrm{~cm}$ antenna separations. They observe that the measured rms delay spreads are on the order of a few tens of ns in the worst case and it and follow a normal distribution.

For UWB signals, multipath components (LoS and NLoS) are generally resolvable since the delay spread is larger than the inverse of larger bandwidths. On the other hand, the power delay profile (PDP) measures the intensity of a signal received through a multipath channel as a function of the time delay. In [185], the PDP of UWB channels is modeled as

$$
p(\Delta \tau)=\alpha(\Delta \tau)^{\beta}+\gamma[\mathrm{dBm}]
$$

where $p$ is the signal power for each individual ray, $\Delta \tau$ is the excess delay with reference to the earliest arrival in ns, and $(\alpha, \beta, \gamma)$ are the fitting parameters. Fitting parameters based on measurements conducted along body-front and body-back are found to be $(-28.58,0.431,-1.88)$ and $(-27.33,0.492,0.63)$, respectively. This can be interpreted as around $25 \mathrm{dBm}$ signal strength loss over 1 ns delay duration. On the other hand, fitting parameters based on measurements conducted along front-torso and back-torso are found to be $(-5.13,0,-24)$ and $(-7.79,0,-22.43)$, respectively. That is, the PDP along the 
torso follows a linear behavior. The RMS and average PDP for in-body UWB links is studied in [171] where the RMS is shown to be less than $1 \mathrm{~ns}$ and the average PDP ranges from $-5 \mathrm{~dB}$ to $-30 \mathrm{~dB}$.

Both delay spread and power delay profile determine the number of channel taps (i.e., significantly resolvable signal paths) and the presence of inter-symbol interference (ISI). Unlike the NB channels, which are well approximated by a single tap [167], UWB channels are described by multiple taps as they are almost 50 times wider than NB channels specified by IEEE 802.15.6 [60]. Therefore, ISI has been mostly observed for UWB on-body channels [191], [199].

2) Level-Crossing Rate and Average Fade Duration: The level-crossing rate (LCR) quantifies the rate at which the signal strength crosses a threshold, particularly at the mean path loss and usually in the positive-going direction. Thus, the LCR can be interpreted as a measure of the rapidity of the fading and often used to determine the Doppler spread as it is a function of the maximum Doppler shift. On the other hand, the average fade duration (AFD) is the average time the received signal is below a threshold and often used to measure the time during which packet losses occur on a link. Therefore, a longer AFD is not acceptable especially by URLLC applications. For a particular threshold value, the product of the average fade duration and the level crossing rate is a constant. Since they are closely related to speed and amount of body movement, they are both essential to characterize the channel dynamics [198].

In [186], the LCR and AFD were evaluated over the 3000 channel samples for threshold values ranging from $-30 \mathrm{~dB}$ to $10 \mathrm{~dB}$, which corresponds to 35 seconds of measurement. The LCR and AFD have been shown for links from center waist to a) head, b) left-arm, c) left-hand, d) and chest. The measurements shows that $\mathrm{LCR}[\mathrm{Hz}]$ has a bell shape whose peak value (around $8 \mathrm{~Hz}$ ) is attained at $-5 \mathrm{~dB}$ and $-3 \mathrm{~dB}$ for links a-b and c-d, respectively. Interestingly, the skewness and kurtosis of LCR curves also changes significantly with link types, which emphasize the impact of node locations on the channel characteristics. On the other hand, the AFD of all links a-d is around $80 \mathrm{~ms}$ until $-10 \mathrm{~dB}$, which starts increasing exponentially and reach to 1 second at $8 \mathrm{~dB}$ approximately. Similar LCR and AFD measurements are also presented in [200] for various antenna types. On the other hand, the AFD of on-body links is also measured to be higher than $300 \mathrm{~ms}$ [201], which is above the $250 \mathrm{~ms}$ latency requirement of IEEE 802.15.6 [60].

3) Auto-Correlation: Autocorrelation of a time-variant channel determines the channel coherence time over which the channel impulse response is considered to be invariant. Thus, autocorrelation and average fade duration govern the duration of successful packet transmission both together. Therefore, they are key metrics to determine packet lengths, channel estimation pilot intervals, and frequency of updates to the power control [198]. While the coherence time of channel during daily activities have been reported to be up to one second, it reduces to $25-70 \mathrm{~ms}$ in case of significant and continuous body movement [198], [202]. The coherence time and coherence bandwidth are closely related and both determine the frequency-selective fading phenomenon. In [29], authors roughly estimate the coherence bandwidth as $30 \mathrm{MHz}$ based on 33 ns delay in a 5 meters long room.

\section{Antenna Effects}

The antenna specifications at both transmitter and receiver side have a considerable impact on the overall channel gain. Unlike free space, the lossy dielectric nature of the human body alters the antenna features by shifting the resonant frequency, detuning impedance matching, modifying radiation pattern, and eventually reducing the overall efficiency [113]. Therefore, the measurements and statistical inferences provided by empirical studies must also consider the impact of antenna type and orientation on the measured attenuation levels.

For on-body links, there are two crucial requirements on antennas: as a natural result of human body's influence on the antenna's reactive field, the distance between antenna and body inherently determines the antenna matching attributes. Therefore, the first antenna requirement is being insensitive to the proximity of the human body. As discussed in Section III-A the energy absorption and skin penetration depth reduces at high frequency ranges, which yields distinct body-antenna interactions at NB and UWB channels. In the NB case, the dominant and major drawback is strong mismatch caused by the resonance frequency shift, which destroys the overall antenna efficiency. A widely accepted approach to overcome this issue is employing adaptive impedance matching [203][205]. On the contrary, the antenna proximity to the body slightly improves the impedance matching at UWB channels for two reasons: The losses help the matching generally by lowering the $S_{11}$ parameter and the high permittivity tissues shifts the band down [206].

The second antenna requirement is having an optimal radiation pattern to minimize attenuation. In addition to antenna specifications, we previously explained that the channel quality is mainly determined by link geometry and antenna positions which determines the propagation mode (LoS, NLoS, creeping wave, etc.). In all these modes, the radiation pattern should be designed to minimize the propagation through-and-off body while maximizing the coupling between body-worn devices [29]. Such a design is non-trivial especially if one considers the large number of link geometry and body posture combinations.

Since the position or orientation of implant IoB cannot be controlled, omnidirectional in-body antennas are generally preferred to directional antennas to establish a reliable communication with a sensor array located on-or-off body [179]. For body-worn antennas, the radiation pattern must be omnidirectional in the horizontal plane and the polarization has to be vertical with respect to the human body [207]. For in-on-and-around the human body communications, a wide variety of antenna types are investigated such as loop antennas, monopole/dipole antennas, and patch antennas [29], [173], [206], [207]. In addition to link loss related issues, there are also other design parameters including endurance, miniaturization, low-profile, low-cost, light-weight, flexibility, comfortability, and ease of fabrication. 


\section{Summary and Insights}

In this section, we begin with the FOCS which are mostly dealing with the mean and variance of the channel attenuation. The FOCS is further classified into two types: large-scale and small-scale fading. The mean and variance of the large-scale fading is mainly characterized by the path loss and shadowing, respectively. We presented five different parametric model which are commonly used to formulate composite path loss and shadowing effects. Accordingly, we tabulated in-body and on-body parametric path loss models in Table $\mathrm{V}$ and Table VI. respectively. One can observe from Table $\mathrm{V}$ that most of the developed models are based on numerical phantoms and computational EM tools presented in Section III-B and Section III-C, respectively. Although IEEE 802.15.6 determines the MICS band as the main channel for implant IoB nodes, there are also a considerable amount of works investigating UWB channels for in-body communications. On the other hand, on-body channels are mostly statistically characterized based on indoor measurements in general. A common observation across all works listed in Table $\mathrm{VI}$ is the discrepancies between reported values under various measurement setting. This is mainly because of the fact that on-body links can be in different propagation modes (LoS, NLoS, creeping wave) which significantly varies with possibly large number of node locations and body postures combinations. Although reported works record conflicting parameter values, almost all of them share common parametric path loss models combined with log-normal shadowing. Unlike the in-body and HBC channels, the on-body NB and UWB links propagate in the air while being in interaction with the surrounding environment. Therefore, small-scale (multi-path) fading is another important channel attributed that has been studied quite extensively. Lognormal, Weibull, and gamma distributions have been found to be best fit, which contradicts with the regular RF channels (offbody) that are generally characterized by Rayleigh, Rician, and Nagakami- $m$ fading.

Since the FOCS are not sufficient to capture the time-variant nature of human mobility, we also delve into the SOCS to shed light into the various communication phenomena, including delay spread, power delay profile, level crossing rate, average fade duration, channel coherence time and bandwidth, and auto-correlation. These are crucial statistics for cross-layer optimization of the IoB network architecture under dynamic channel conditions. Lastly, we pointed out the impacts of antenna features on the channel attenuation measurements. Indeed, antenna radiation pattern and its proximity to the body has significant influence on shifting resonant frequency, detuning impedance matching, modifying radiation pattern, and finally reducing the overall efficiency. Therefore, it is necessary to quantify antenna gain along with other factors affected by the measurement set-up.

\section{HBC Channel Modeling}

In previous section, we delved into the channel characteristics of in/on body wireless links where NB and UWB signals propagates in/around the human body. $\mathrm{HBC}$ is an alternative and promising wireless approach that uses human skin tissues as a communication channel, thus it is also referred to as body channel communication (BCC) or intra-body communication (IBC). HBC couples communication signals to the body through electro-static or magneto-static field via transmitter electrodes, which are captured by receiver electrodes on another part of the body. It is worth noting that such a communication is possible due to the bio-electromagnetic properties of the human body, which is covered in Section III-A

The HBC channel is specifically confined to frequency band between $100 \mathrm{kHz}$ and $100 \mathrm{MHz}$ for two reasons [30]: 1) Frequencies lover than $100 \mathrm{kHz}$ is susceptible to all sort of EM interference, and 2) At frequencies above $100 \mathrm{MHz}$, the human body acts as an antenna since the carrier wavelength reaches to the length of human body parts, where there is no longer HBC. This yields a carrierless communication scheme and decouples the transceiver size from the carrier wavelength. Operating on such low frequencies also have advantages in terms of modem complexity, power efficiency, physical layer security, safety, and so on.

In this section, we first provide an overview of three main coupling methods: galvanic coupling, capacitive coupling, and magnetic coupling. After that, we focus on channel characterization techniques which are based on analytical, numerical, circuit, and empirical models. Finally, we discuss how HBC channels are affected by human body movements and dynamics of the surrounding environment.

\section{A. An Overview of Coupling Methods}

1) Capacitive Coupling (CC): The capacitive coupling, also known as near-field coupling or electro-static coupling, was first proposed for personal area networks by Zimmerman in 1995 [208]. The CC is illustrated in Fig. 9 where signal electrodes of both transmitter and receiver are attached to the skin whilst the ground electrodes are kept floating in air. The stimulated electric field on the signal electrode of the transmitter induces an electric flow on the signal path through the human body and a external return path through the surrounding environment (i.e., air). In other words, the conductivity of body not only generates the forward path but also creates a backward path by coupling electric field to the environment, the earth ground, and electrodes' ground plates [38]. For this very reason, the $\mathrm{CC}$ is more sensitive to environmental changes (e.g., appearance of nearby metallic objects and wires) and susceptible to interference caused by devices radiating electric fields.

2) Galvanic Coupling $(G C)$ : The galvanic coupling was first demonstrated in [209] where Handa et. al. coupled the modulated ECG signals to the human chest, that are received by a pair of electrodes on the wrist. The GC is illustrated in Fig. 10 where both signal and ground electrodes of transmitter and receiver are in contact with skin. On the contrary to $\mathrm{CC}$, the GC is independent of environmental affects since the signal is mostly confined within the human body. GC is suitable for short distances $(\sim 15-40 \mathrm{~cm})$ and low frequencies $(<1 \mathrm{MHz})$, which restricts its suitability for QoS demanding applications [30]. Its stable and reliable channel 


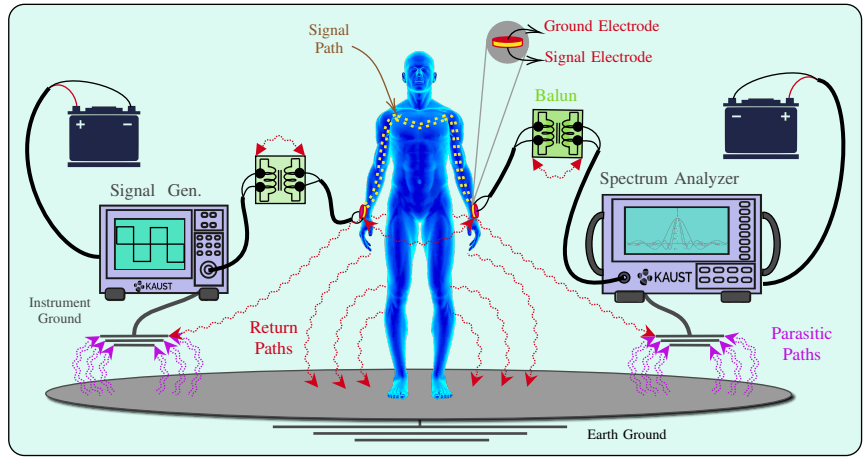

Fig. 9: Illustration of capacitive coupling based HBC.

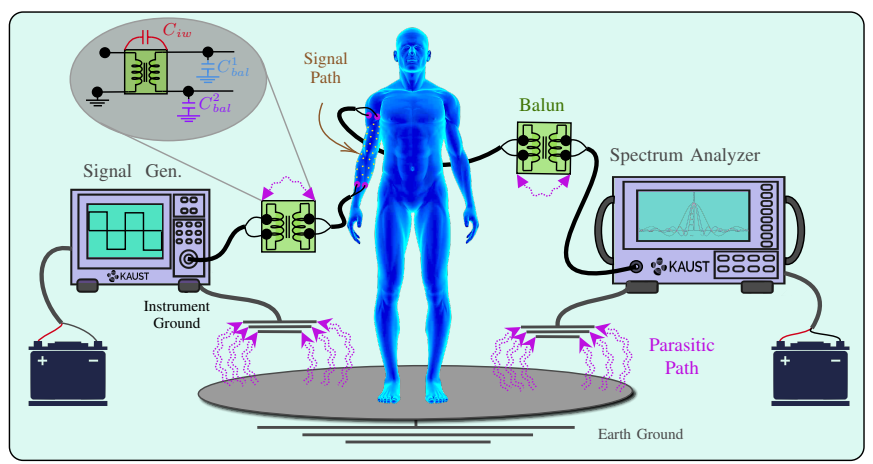

Fig. 10: Illustration of galvanic coupling based HBC.

conditions make the GC is a good option to periodically transmit physiological data especially using devices embedded underneath the skin [38].

3) Magnetic Coupling (MC): Unlike CC and GC, magnetic coupling exploits the electromagnetic resonance to generate a magneto quasi-static field throughout the body. As demonstrated in Fig. 11, the current induced by the signal flowing through the transmitter's coil (yellow dashed lines) generates a magnetic field (red dashed lines). When the foot and hand touch the ground and signal electrode at the same time, respectively, the conductive line and human body create a communication channel by forming an effective loop (green dashed lines) [210]. Notice that the communication channel disappears if foot or hand is detached from the electrodes. That is, the communication channel is connected and disconnected by closing and opening the effective loop, respectively. Similar to $\mathrm{CC}, \mathrm{MC}$ can reach relatively long distances $(\sim 120 \mathrm{~cm})$.

\section{B. HBC Channel Models}

An accurate channel model is essential for developing effective HBC systems. Nonetheless, this is a non-trivial task as $\mathrm{HBC}$ channels are affected by many factors such as environment, distance between transceivers, electrode orientation and locations on the body, variable contact impedance of different electrode types and their specifications, and backward loss that dynamically changes with various body postures and movements.

1) Analytical Models: As discussed in Section IIII-B and Section III-C, Maxwell's equations can explain the electric field within and around the body through a set of complex

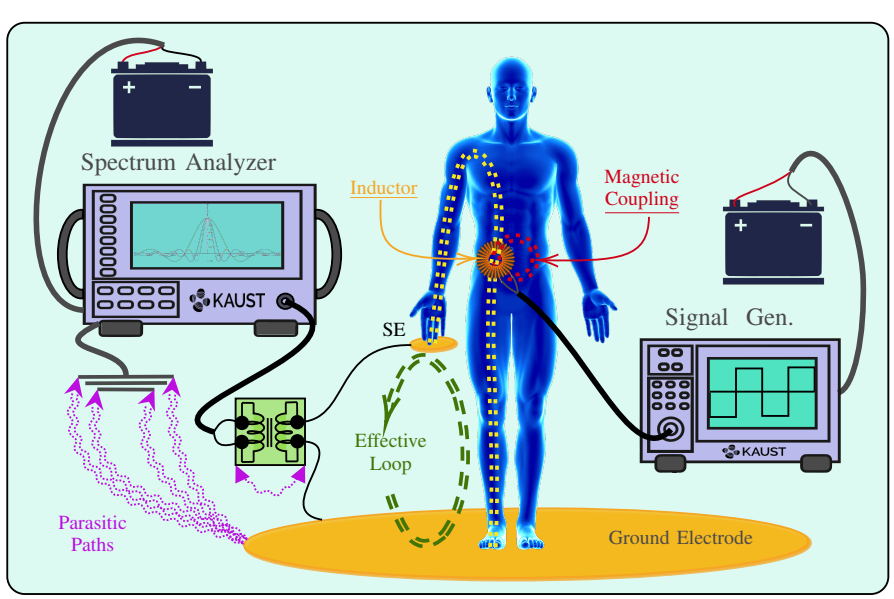

Fig. 11: Illustration of magnetic coupling based HBC.

TABLE VII: Analytical and Numerical HBC channel models.

\begin{tabular}{|c|c|c|c|c|c|c|c|}
\hline & & Ref. & Year & Coup. & Frequency & Pathloss & Validation Methods \\
\hline \multirow{4}{*}{\multicolumn{2}{|c|}{ 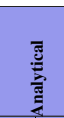 }} & 211] & 2009 & $\mathrm{GC}$ & $1 \mathrm{KHz}-1 \mathrm{MHz}$ & Linear & SIM: Finite element Mesh \\
\hline & & 212 & 2012 & $\mathrm{CC}$ & $1 \mathrm{KHz}-100 \mathrm{MHz}$ & Linear & $\begin{array}{l}\text { EXP: In-VIVo measure. } \\
\text { EXP: Measure. up to } 1.3 \text { meters. }\end{array}$ \\
\hline & & - & & & & & SIM: FDTD simulations \\
\hline & & [213] & 2016 & GC & $<5 \mathrm{MHz}$ & Linear & EXP: Liquid Muscle Tissue Phantom \\
\hline \multirow{11}{*}{ 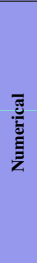 } & \multirow{5}{*}{$\sum_{\substack{x \\
\Gamma}}$} & 149) & 2009 & $\mathrm{CC}$ & $1 \mathrm{MHz}-100 \mathrm{MHz}$ & & EXP: In-vivo measure. (VNA) \\
\hline & & 150 & 2011 & $\mathrm{CC}$ & $1 \mathrm{MHz}-100 \mathrm{MHz}$ & Linear* & EXP: In-vivo measure. (VNA) \\
\hline & & 151 & 2012 & GC & $10 \mathrm{kHz}-5 \mathrm{MHz}$ & Linear* & EXP: In-vivo measure. (SG+OS) \\
\hline & & 152 & 2014 & GC & $1 \mathrm{KHz}-100 \mathrm{MHz}$ & Linear & EXP: In-vivo measure. (SG+OS) \\
\hline & & 153 & 2017 & $\mathrm{CC}$ & $1-40 \mathrm{MHz}$ & Linear* & EXP: In-vivo measure. (VNA w. Balun) \\
\hline & \multirow{6}{*}{$\hat{\hat{\theta}}$} & 154 & 2018 & GC & $<1 \mathrm{MHz}$ & Linear* & EXP: In-vivo measure. (SG+OS w. Balun) \\
\hline & & 1621 & 2003 & GC & & & \\
\hline & & $\frac{1001}{104 \mid}$ & $\begin{array}{l}2004 \\
2006\end{array}$ & $\begin{array}{l}\text { with } \\
\text { single }\end{array}$ & $10 \mathrm{MHz}$ & - & EXP: Tissue-equivalent solid phantom \\
\hline & & 165 & 2007 & elect. & & & \\
\hline & & 100 & 2009 & pair & $10-100 \mathrm{MHz}$ & Linear & EXP: In-vivo measure. (SG+OS) \\
\hline & & & tor $\mathrm{N}$ & & & 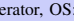 & Iscilloscope, *: Freq. dep. pathloss \\
\hline
\end{tabular}

electric field equations. Therefore, analytical models have been developed to investigate the electric field based on electromagnetic theories [166], [211]-[213]. In [212], authors consider three main electric field components of CC-HBC; the quasistatic near field, induction-field radiation, and the surface wave field. The theoretical analysis is validated by measurements up to frequency of $100 \mathrm{MHz}$ and channel distance of $1.3 \mathrm{~m}$. A linear pathloss model (Model A in (4)) is also presented and compared with FDTD based linear model given in [166]. In [213], authors exploit multilayered ellipsoidal geometry to present a unified analytical GC-HBC channel model that can be used for any part of the body. The model is validated by FDTD simulations and phantom measurements. Similar to other works, pathloss has been shown to increase linearly with frequency and distance.

Wegmueller et. al. investigate GC-HBC by using finite element method to understand the impacts of distance, tissue types, electrode positions, and joints on signal attenuation between $1 \mathrm{kHz}$ and $1 \mathrm{MHz}$ [211]. The developed analytical models were compared with numerical simulations and invivo clinical trials. The results showed that a $5 \mathrm{~cm}$ increase in distance yields 6-9 $\mathrm{dB}$ more path loss while joints may put an additional $8 \mathrm{~dB}$ loss proportional to their size. Although receiver electrode size had a negligible effect, a larger transmitter size yielded a lower attenuation. The resistance of different tissues was also shown to have varying influence on the path loss. 
TABLE VIII: Circuit models.

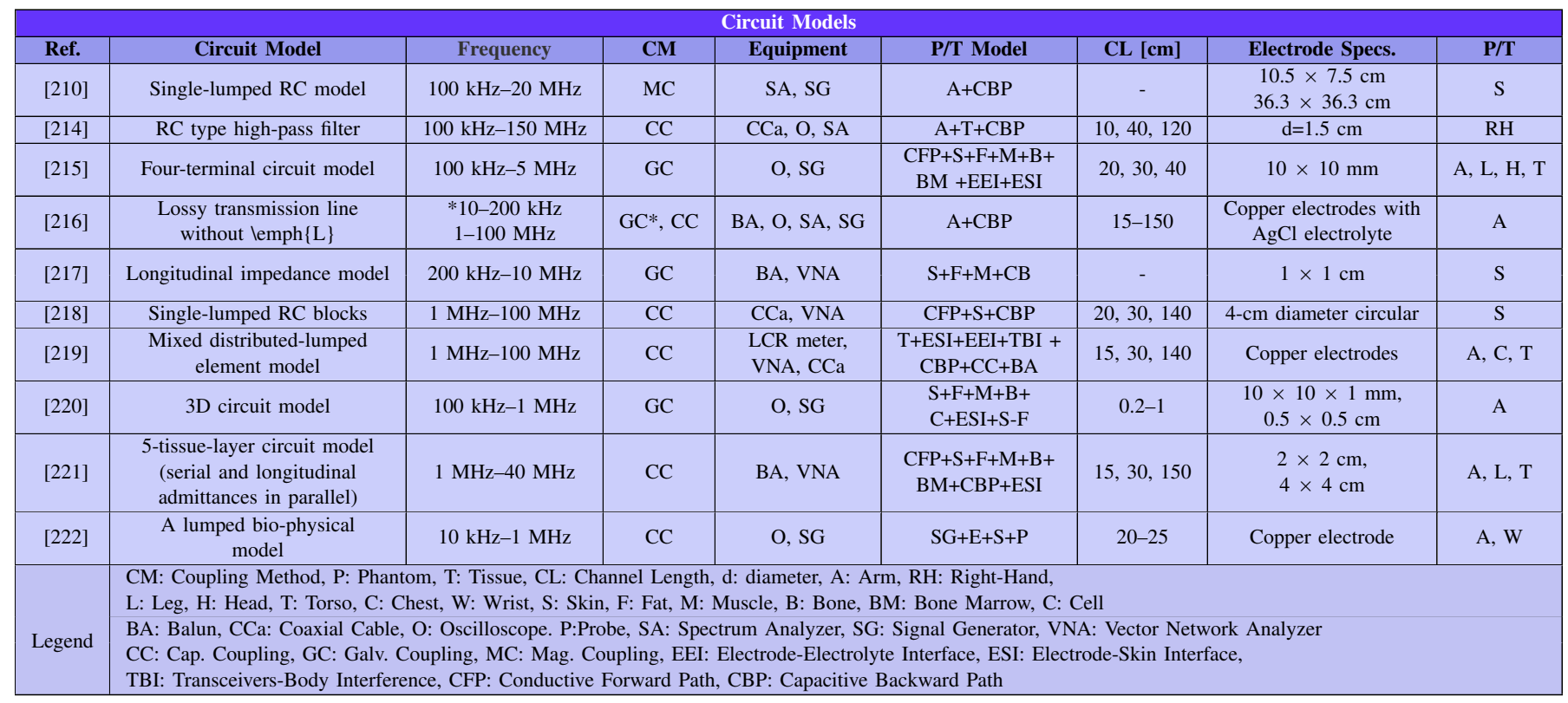

2) Numerical Models: Among numerical tools presented Section III-C, there are two common methods used to simulate the signal propagation on HBC channels: FEM [149]-[154] and FDTD [162]-[166]. We refer interested readers to Section III-C for technical details of FEM and FDTD.

FEM: In [149], a circuit-coupled FEM method is used to explore CC-HBC. The FEM simulations on multilayer human forearm model is validated by clinical trials by using VNAs between 1-100 MHz. Results showed that increasing path loss by distance is mainly caused by the increasing length of the parasitic capacitor return path. The work in [149] is extended in [150] by dividing the surrounding environment into three regions: near-field region, transmission region, and far-field region. A whole body FEM simulations are conducted for GC$\mathrm{HBC}$ in [151] where frequency-dependent signal attenuation has exhibits different slopes in low and high frequency ranges. Moreover, the impact of distance on the frequency-dependent signal attenuation was also observed to become more significant as the frequency increases. A GC-HBC is also considered in [152] where the influence of frequency, channel length, and inter-electrode distance have been analyzed. Specifically, authors have shown that attenuation increases as far as 20 $\mathrm{dB}$ for an increment of $5 \mathrm{~cm}$ and concluded that $\mathrm{GC}$ is limited to short distances. In [153], CC-HBC is investigated with different ground electrode heights, separation distances, and dimensions. During our initial investigations using FEM simulators, we observe that path loss exponentially decays with increasing frequency while its relation with distance is linear. We refer interested readers to [223] for a deeper insight into the FEM based analysis and estimation of the GC-HBC channels.

Furthermore, the effects of different shapes and relative angles of the ground electrodes are investigated for the first time. The results have shown that a shape with more sides yields a smaller path loss. Interestingly, authors conclude their simulation and experimental results with a path loss model which depends on air and ground coupling capacitances and electrode angles. In [154], authors provide a computational GC-HBC analysis covering some issues that was not fully explained before, including the modeling of skin-electrode impedance, the differences associated with the use of constant voltage, current excitation modes, the influence of the subject's bioelectric properties on both distance and frequency dependent attenuation.

FDTD: In [162]-[165], authors employs FDTD method for GC-HBC where a single pair of electrode is considered such that signal and ground electrodes act as transmitter and receiver, respectively. The simulation results are validated with measurements on tissue-equivalent solid phantom. [166]

3) Circuit Models: Previously discussed numerical models have two main drawbacks: a long simulation time and accuracy limited to low-frequency approximations of Maxwell's equations. Alternatively, circuit models offer shorter running times and accuracy for a wide range of frequencies. The circuit models are established on simple transfer functions that mathematically characterize the signal propagation along a transmission path on/in the human body [210], [214]-[222].

By using the frequency-dependent dielectric properties of different tissue types, a simple phantom can be modeled with resistance and capacitance, representing the dissipation loss and charge holding ability of tissues, respectively. Since the human body parts are small compared to signal wavelengths under $100 \mathrm{MHz}$, the lumped-element model is capable of analyzing the signal behavior [221]. Accordingly, a variety of approaches have been developed such as the use of a single RC model [210], longitudinal impedance model [217], 3D circuit model [220], and 5-tissue-layer circuit model [221], etc. Table VIII compare the circuit models based on frequency range, coupling method, equipment used for measurement, employed phantom/tissue model and types, propagation distance, and electrode specifications. The range of frequencies of interest 
and the coupling method are the main considerations to decide on the model that fits best the channel. To date, there is no standardized circuit model that characterizes the full human body over the entire HBC frequency range. This is mainly because of deteriorating accuracy level when generalization of a circuit model is not capable of accounting for various practical issues, which are discussed in the sequel.

The accuracy of the circuit models can be affected by several factors such as conductive forward body path (CFP); capacitive backward path (CBP); propagation distance; dielectric properties of tissue layers; the signal radiation leaked to the air and absorbed by body tissues; and electrodeskin contact/interface impedance. These effects are generally classified as intrinsic and extrinsic. The accuracy of a circuit models lies within the ability to account for intrinsic and extrinsic effects in the transfer functions. The intrinsic effects can be considered as static as they merely depend on channel distance and tissue properties along the propagation path rather than external/environmental conditions. On the contrary, the extrinsic effects are caused by the return and parasitic paths, as shown in Fig. 9 and Fig 10 . Furthermore, the interface between electrodes and skin is a significant part of the extrinsic channel as well as skin conditions such as age and moisture level of the skin [221]. Based on above discussion, one can tell that the intrinsic and extrinsic effects are more related to CFP and $\mathrm{CBP}$, respectively. Indeed, one common observation on the presented models by references tabulated in Table VIII is that the accuracy decreases at higher frequency ranges, especially above $70 \mathrm{MHz}$.

4) Empirical Models: A common shortcoming of the previous channel models is that they cannot capture the impacts of environmental effects on the channel model. As a remedy, empirical models can provide a deeper insight into the channel behaviors under real circumstances. Fig. 9.Fig. 11 depict different measurement setup by using various equipment such as signal generator (SG), vector network analyzer (VNA), and spectrum analyzer (SA). However, recent studies have revealed that empirical studies reported significantly inconsistent channel gains due to different measurement configurations in terms of grounding, load resistance, the effect of cables and connections, and type of measurement devices [210], [214], [224]-[226], [234], [235]. We list the empirical studies in terms of these configurations in Table $\mathrm{IX}$ and discuss details in what follows.

a) Grounding Effects: Using earth-grounded equipment leads to erroneous channel gain measurements since there is a cabled path between the measurement devices' internal grounds. In such a case, the true channel gain is overestimated as the measurement instruments' ground plane creates a larger path loss than the actual return path. In order to avoid such optimistic measurements, TX and RX ports have often been isolated from the common internal ground by using balancedto-unbalanced (balun) components. In [222], the channel characteristic without baluns shows a flat band response with a loss of around $20 \mathrm{~dB}$ over the frequency range of $100 \mathrm{KHz}-100$ $\mathrm{MHz}$. On the other hand, introducing baluns show a band-pass characteristic with a loss of $\sim 80 \mathrm{~dB}$ around $100 \mathrm{KHz}$ and the minimum loss of $\sim 20 \mathrm{~dB}$ around $35 \mathrm{MHz}$. However, the use of baluns cause two types of parasitic paths [235]:

- As shown in Fig. 10, parasitic paths goes through interwinding capacitance $\left(\mathrm{C}_{i \omega}\right)$ that occur between balun terminals.

- The differential signal influence the HBC channel by forming a return path through the surrounding environment. Thus, the asymmetrical capacitance between the ground and balun terminals should be the same in the whole frequency range. In this way, only parasitic paths from instrument's ground to the earth's ground will remain, as shown in Fig. 9 and 10

Nonetheless, the use of baluns is not a complete solution to the grounding effects. In [224], it has been shown that inclusion of any additional ground plane area overestimates the channel gain by up to $33.6 \mathrm{~dB}$, whether it is isolated by via a balun or not. Therefore, recent studies considered batterypowered transceivers/instruments for a more accurate channel characterization [216], [219], [234].

b) Load Resistance and Instrument Effects: Another critical concern on the experimental configuration is the input resistance of measurement instruments and transceivers. In the literature, measurement campaigns typically used $50 \Omega$ and $1 \mathrm{M} \Omega$ load resistances [236]. Especially in the case of $\mathrm{GC}-\mathrm{HBC}$, the impedance observed before and after the instruments is the same as that presented by the human body and electrode interface, which is independent of the frequency [152]. Therefore, $50 \Omega$ may not be the best option for channel characterization. A comparison between $50 \Omega$ and $1 \mathrm{M} \Omega$ load resistances shows that $1 \mathrm{M} \Omega$ yields a $25 \mathrm{~dB}$ more channel gain at $10 \mathrm{KHz}$, which reduces to $15 \mathrm{~dB}$ at $1 \mathrm{MHz}$ [234]. It is also worth noting that VNAs, SGs, SAs, and oscilloscopes have different characteristics and thus different impacts on the measurements. In light of the above discussions, achieving optimum coupling is possible only if any impedance mismatch is avoided by taking the aforementioned issues into account.

c) Cable Effects: The electrical cables are usually prone to attenuation and radiation at higher frequencies, which limits the range of study of the HBC channels. Therefore, their effect should be minimized as much as possible by using correct matching and minimal length. In the literature, several studies explored the cable effects [214], [218], [224]-[227], [230], [231], [234]. Various cable types are compared over $10 \mathrm{kHz}$ to $100 \mathrm{MHz}$ frequency range in [234], where the performance of computational and experimental results match well up to 1 $\mathrm{MHz}$, then a difference about $10 \mathrm{~dB}$ observed starting from 4 $\mathrm{MHz}$.

Excluding [226], the measurement campaigns carried out in references listed in Table IX with a limited number of participants, even some of them consider only a single human subject. Since this inherently yield statistically insignificant results [225], [227]. To the best of authors' knowledge, a holistic cross-sectional and longitudinal study is yet to be performed to reach statistically meaningful inferences on the channel characteristics.

\section{Variable Electrode-Skin Impedance (ESI)}

The electrode-skin (contact) impedance (ESI) between the human body and the signal electrode has a considerable 
TABLE IX: Empirical models.

\begin{tabular}{|c|c|c|c|c|c|c|c|c|c|c|c|c|c|c|c|}
\hline \multicolumn{16}{|c|}{$\begin{array}{c}\text { Empirical Models } \\
\end{array}$} \\
\hline Ref. & VNA & SA & SG & $\mathbf{O}$ & B & $\begin{array}{c}\text { Cable } \\
\text { Effects }\end{array}$ & $\begin{array}{c}\text { Battery } \\
\text { Powered }\end{array}$ & $\begin{array}{c}\text { Ground } \\
\text { Plane }\end{array}$ & $\begin{array}{c}\# \\
\text { People }\end{array}$ & $\begin{array}{c}\text { Env. } \\
\text { Effects }\end{array}$ & $\begin{array}{l}\text { Body } \\
\text { Mov. }\end{array}$ & Electrode & CM & Frequency & $\begin{array}{c}\text { Channel } \\
\text { Length }\end{array}$ \\
\hline 214] & $\mathrm{N}$ & $\mathrm{Y}$ & - & $\mathrm{Y}$ & $\mathrm{N}$ & $\mathrm{Y}$ & $\mathrm{Y}$ & $24-60 \mathrm{~cm}^{2}$ & 1 & $\mathrm{Y}$ & $\mathrm{Y}$ & $\mathrm{d}=1.5 \mathrm{~cm}$ & $\mathrm{CC}$ & $100 \mathrm{kHz}-150 \mathrm{MHz}$ & $10,40,120 \mathrm{~cm}$ \\
\hline 224 & $\mathrm{Y}$ & $\mathrm{Y}$ & $\mathrm{N}$ & $\mathrm{N}$ & $\mathrm{Y}$ & $\mathrm{Y}$ & $\mathrm{Y}$ & $4 \times 4 \times 3 \mathrm{~m}$ & 1 & - & $\mathrm{N}$ & $30 \times 30 \mathrm{~mm}^{2}$ & $\mathrm{CC}$ & $20-150 \mathrm{MHz}$ & $20 \mathrm{~cm}$ \\
\hline 225] & $\mathrm{Y}$ & $\mathrm{N}$ & $\mathrm{N}$ & $\mathrm{N}$ & $\mathrm{Y} / \mathrm{N}$ & $\mathrm{Y}$ & $\mathrm{Y} / \mathrm{N}$ & $2.4 \times 2.4 \times 3 \mathrm{~m}$ & 11 & $\mathrm{Y}$ & $\mathrm{Y}$ & $2 \times 2 \mathrm{~cm}^{2}$ & GC & 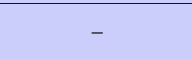 & $\begin{array}{c}4,16,28, \\
36,120 \mathrm{~cm}\end{array}$ \\
\hline 210$]$ & $\mathrm{N}$ & $\mathrm{Y}$ & $\mathrm{Y}$ & $\mathrm{N}$ & $\mathrm{N}$ & - & $\mathrm{N}$ & $36.5 \times 36.5 \mathrm{~cm}$ & 1 & $\mathrm{~N}$ & $\mathrm{Y}$ & $7.5 \times 10.5 \mathrm{~cm}^{2}$ & MC & $\leqslant 10 \mathrm{MHz}$ & \\
\hline 226 & $\mathrm{~N}$ & $\mathrm{~N}$ & $\mathrm{Y}$ & $\mathrm{Y}$ & $\mathrm{N}$ & $\mathrm{Y}$ & $\mathrm{N}$ & $7 \times 4 \mathrm{~cm}^{2}$ & 94 & - & $\mathrm{N}$ & $2 \times 2 \mathrm{~cm}^{2}$ & $\mathrm{CC}$ & $\leqslant 100 \mathrm{MHz}$ & $1.5 \mathrm{~m}$ \\
\hline 227] & $\mathrm{Y}$ & $\mathrm{N}$ & $\mathrm{N}$ & $\mathrm{N}$ & $\mathrm{Y}$ & $\mathrm{Y}$ & - & - & 4 & $\mathrm{Y}$ & $\mathrm{Y}$ & $\begin{array}{l}3 \times 3 \mathrm{~cm}^{2} \\
6 \times 8 \mathrm{~cm}^{2}\end{array}$ & & $300 \mathrm{kHz}-15 \mathrm{MHz}$ & $30,90,150 \mathrm{~cm}$ \\
\hline 218 & $\mathrm{Y}$ & $\mathrm{N}$ & - & - & $\mathrm{Y}$ & $\mathrm{Y}$ & $\mathrm{N}$ & - & 1 & - & $\mathrm{N}$ & $2 \times 2 \mathrm{~cm}^{2}$ & $\mathrm{CC}$ & $1-100 \mathrm{MHz}$ & $5<\mathrm{d}<150 \mathrm{~cm}$ \\
\hline 228] & $\mathrm{Y}$ & $\mathrm{N}$ & $\mathrm{Y}$ & - & $\mathrm{N}$ & $\mathrm{N}$ & $\mathrm{N}$ & - & 1 & - & $\mathrm{N}$ & $\begin{array}{l}2 \times 5 \mathrm{~cm}^{2} \\
2 \times 10 \mathrm{~cm}^{2}\end{array}$ & & $1 \mathrm{MHz}-2.5 \mathrm{GHz}$ & $0.7<\mathrm{d}<180 \mathrm{~cm}$ \\
\hline 229$]$ & $\mathrm{Y}$ & $\mathrm{N}$ & $\mathrm{Y}$ & $\mathrm{N}$ & $\mathrm{N}$ & - & $\mathrm{N}$ & - & 1 & - & $\mathrm{Y}$ & $\mathrm{d}=1 \mathrm{~cm}, 3 \mathrm{~cm}$ & $\mathrm{CC}$ & $200-600 \mathrm{MHz}$ & $20<\mathrm{d}<155 \mathrm{~cm}$ \\
\hline 230 & $\mathrm{Y}$ & $\mathrm{N}$ & $\mathrm{N}$ & $\mathrm{N}$ & $\mathrm{Y}$ & $\mathrm{N}$ & $\mathrm{N}$ & - & 1 & $\mathrm{~N}$ & $\mathrm{~N}$ & $2 \times 2 \mathrm{~cm}^{2}$ & & $10-100 \mathrm{MHz}$ & $15 \mathrm{~cm}$ \\
\hline 231] & $\mathrm{Y}$ & $\mathrm{N}$ & $\mathrm{N}$ & $\mathrm{Y}$ & $\mathrm{Y}$ & $\mathrm{Y}$ & $\mathrm{N}$ & - & 1 & $\mathrm{~N}$ & $\mathrm{~N}$ & $\begin{array}{l}4 \times 5 \mathrm{~cm}^{2} \\
4 \times 10 \mathrm{~cm}^{2}\end{array}$ & $\mathrm{CC}$ & $1-100 \mathrm{MHz}$ & $20 \mathrm{~cm}$ \\
\hline 232 & $\mathrm{~N}$ & $\mathrm{~N}$ & $\mathrm{~N}$ & $\mathrm{~N}$ & $\mathrm{~N}$ & $\mathrm{Y}$ & $\mathrm{Y}$ & - & 1 & $\mathrm{Y}$ & $\mathrm{Y}$ & $4 \times 4 \mathrm{~cm}^{2}$ & & $100 \mathrm{kHz}-60 \mathrm{MHz}$ & $11 \mathrm{~cm}$ \\
\hline 211] & $\mathrm{N}$ & $\mathrm{N}$ & $\mathrm{N}$ & $\mathrm{N}$ & $\mathrm{N}$ & $\mathrm{N}$ & $\mathrm{Y}$ & - & 2 & $\mathrm{~N}$ & $\mathrm{~N}$ & $\begin{array}{r}28 \times 20 \mathrm{~cm}^{2} \\
30 \times 22 \mathrm{~mm}^{2} \\
\end{array}$ & GC & $10 \mathrm{kHz}-1 \mathrm{MHz}$ & 5 and $7 \mathrm{~cm}$ \\
\hline 233 & $\mathrm{~N}$ & $\mathrm{Y}$ & $\mathrm{Y}$ & $\mathrm{N}$ & $\mathrm{N}$ & $\mathrm{N}$ & $\mathrm{Y}$ & - & 1 & $\mathrm{Y}$ & $\mathrm{Y}$ & $4 \times 4 \mathrm{~cm}^{2}$ & $\mathrm{CC}$ & - & - \\
\hline Legend: & VNA & & & & & & & & & & & & & & \\
\hline
\end{tabular}

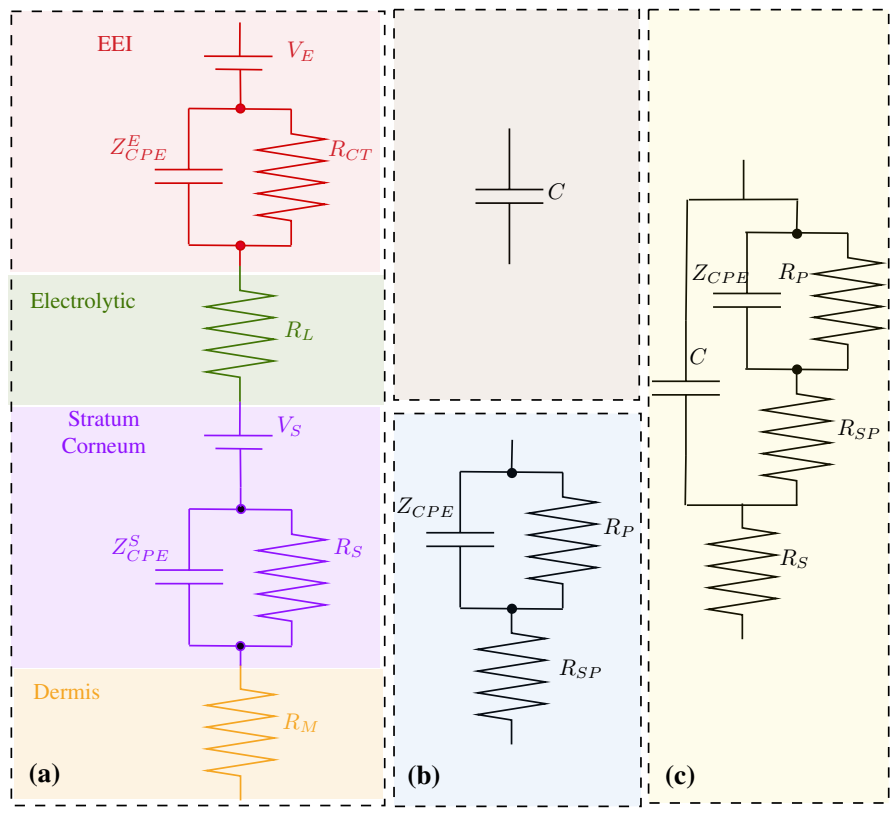

Fig. 12: Equivalent circuit model of the ESI.

impact on the overall path loss. The contact impedance is determined by the electrode-electrolyte interface (EEI) and the skin-surface impedance (SSI) track. While the EEI is characterized by electrode types (wet or dry) and specifications (size, shape, metal), the SSI varies with operating frequency, skin conditions, and body motions. Therefore, the channel gains change with the contact impedance variations, which characterize the shadowing effects on HBC channels. In this subsection, we first introduce the circuit representation of ESI components and then discuss the impacts of electrode types (wet or dry) and specifications (size, shape, metal) on the contact impedance.

1) ESI Circuit Representation: The human skin consists of three main layers; stratum corneum (SC), epidermis, dermis, which have the following electrical properties [237]: since the SC mostly consists of dead cells, it is has the largest impedance especially under $1 \mathrm{kHz}$. The epidermis and dermis have similar water content (40\%-60\%) and electrical properties, and thus form the viable skin, that contributes to skin impedance especially above $1 \mathrm{MHz}$.

Based on this vertically layered skin structure, the ESI equivalent circuit model is illustrated in Fig. $12 \mathrm{a}$ a, where the EEI is represented by the electrical potential difference between electrode and electrolyte $\left(V_{E}\right)$, charge transfer resistance accounting for the Faraday effect $\left(R_{C T}\right)$, and electrode constant phase element (CPE) accounting for the non-faradic characteristics $\left(Z_{C P E}^{E}\right)$ [238]. Likewise, SSI is represented by electrical potential $\left(V_{S}\right)$ and a parallel connection of skin resistance $\left(R_{S}\right)$ and CPE $\left(Z_{C P E}^{S}\right)$. On the other hand, the resistance of sweat/water and dermis is denoted by $R_{L}$ and $R_{M}$, respectively. As shown in the upper part of Fig. $12 \mathrm{~b}$, the non-contacting electrode parts (highlighted in red and green backgrounds) can be simplified to a capacitor as they exhibit a non-faradic process [239]. Moreover, the contacting electrode parts (highlighted in purple and yellow backgrounds) can be reduced to polarization resistance $\left(R_{P}\right)$, the $\mathrm{CPE}\left(Z_{C P E}^{E}\right)$, and electrolyte resistance $\left(R_{S P}\right)$. Finally, the circuit model can be further simplified, as shown in Fig. 12. c where $R_{S}$ represents the superposition of body-wire-and-lead resistances, resulting in a double-order electrode impedance model.

It is worth noting that the above model parameters are affected by polarization and motion effects. The overall impedance should be derived by combining $R_{C T}$ with the polarization at low-frequency ranges [240]. On the other hand, movement effects can lead to changes in $V_{S}$, which is also referred to as motion artifacts and can be mitigated by the use of conductive electrode gels [241]. Furthermore, these parameters also highly dependent on skin conditions and electrode specifications, which are discussed next.

2) Impacts of Electrode Types and Specifications: Similar to the role of antennas in the NB/UWB systems, electrodes have a major impact on the overall channel path loss as they are the main components that interface the human body 
and transceiver. Electrodes generally do not deliver the same performance as they come in a variety of designs, structures, and materials, etc. In what follows, we first present electrode types and then discuss various specifications and their effects on the contact impedance.

a) Electrode Types: Electrodes are broadly classified as wet and dry electrodes based on whether some chemical gels are used to increase the conductivity. The wet electrodes are further sorted into two types: electrolyte and pre-gelled electrodes. The latter offers a good fit and high conductivity and has a wide-spread use thanks to their disposable and low-cost nature [242]. However, the gel conductivity would degrade over prolonged use and/or through sweat/grease accumulation. This inherently deteriorates the overall transducer performance and limits the wet electrodes to single and short-term clinical use [237].

On the other hand, dry electrodes have a large contact impedance as it is a metal directly attached to the skin surface. The lack of a conductive gel causes two main problems: Firstly, the air generally traps between the electrode and skin, which creates an extra dielectric layer and increases the overall impedance considerably. Secondly, the electrode may not attach well to a dry skin surface, which yields motion artifacts and high contact impedance, as mentioned in the previous subsection. To overcome these drawbacks, dry electrodes are recently designed by using soft substrates, such as textile electrodes [243], tattoo electrodes [244], and skinlike electrodes [245] are popular examples of soft-electrodes. Since IoB nodes are generally used for long-term applications, dry electrodes are more suitable in terms of a longer lifetime and reduced discomfort, which are vital attributes for commercialization efforts.

b) Electrode Specifications: Material, size, and geometry of the electrodes are essential for the contact impedance's selectivity and sensitivity. In order to avoid irritation/discomfort to user skin and eliminate the performance deterioration over long-term use, electrode material should be biocompatibility and strike an excellent electrochemical balance at the ESI. Silver/silver chloride $(\mathrm{Ag} / \mathrm{AgCl})$ electrodes are widely accepted since they have stable chemical properties, low compensation voltage, low intrinsic noise, and low ESI [241]. Since $\mathrm{Ag} / \mathrm{AgCl}$ electrodes have a simple and mature fabrication process, they also have a low-cost [237]. In addition to the above $\mathrm{Ag} / \mathrm{AgCl}$ electrode advantages, gold electrodes offer a better biocompatibility conductivity at a higher cost. Furthermore, metallic and carbon-based nanomaterials are also used in electrodes thanks to their advantages of having a large surface, higher conductivity, and better connectivity. For example, AgNW based metal nanoparticle and nanowire electrodes are shown to deliver similar/superior performance with/than $\mathrm{Ag} / \mathrm{AgCl}$ electrodes in resting/moving states [246].

The electrode size and geometry determine effective contact area, sensitivity, depth, and intensity of the applied electric field, and signal-to-noise ratio [247]. Electrodes typically come in rectangular, circular, spiral, ring, and interdigital shapes. While circular and rectangular electrodes are commonly macro-block electrodes, others are often microelectrodes. Along with the electrode size, inter-electrode spacing also has a considerable impact on the penetration depth and electric field distribution [248].

3) Impedance Matching: Throughout the previous subsections, our discussion concludes that modeling ESI is not trivial since it is affected by many factors, which inherently yields design uncertainty and degrades the connection reliability. The variable contact impedance also requires an extra margin at both the transmitter and receiver side, which results in an additional power budget. These necessitate impedance compensation techniques to maximize the power transfer at the electrode-to-skin interface [249], which can be done via matching networks [250]. At the cost of extra power margin, a commonly adopted way is increasing and decreasing the input and output impedance of the receiver and transceiver, respectively [251]. A power-efficient alternative is adaptive and automatic receiver mode selection based on an impedance sensing circuit [252]. In [253], authors develop low and high modes to mitigate the variable contact impedance. The noncontact cases have been considered in [254], [255], where an inductor is employed to compensate for the electrode-skin interface capacitance in a dynamic fashion.

\section{Summary and Insights}

Unlike the NB/UWB channels' coexistence and interference problems, the HBC is an alternative solution with the virtues of ultra low power operation, sufficient throughput for most of the IoB applications, and enhanced physical layer security due to the low signal leakage. In this section, we first outline three main coupling methods: capacitive, galvanic, and magnetic. In the realm of $\mathrm{HBC}, \mathrm{CC}-\mathrm{HBC}$ and $\mathrm{GC}-\mathrm{HBC}$ are mostly preferred due to their practical use. While GC-HBC confines both forward and the backward path to the human body, the $\mathrm{CC}$ HBC completes the backward path through the environment, the earth's ground, and electrodes' ground plates. For this reason, the $\mathrm{CC}-\mathrm{HBC}$ is more sensitive to the environmental changes than the $\mathrm{GC}-\mathrm{HBC}$.

The HBC channels are characterized by analytical, numerical, circuit, and empirical models. Analytical models are important to gain a deep insight into the propagation mechanism by using a set of complex Maxwell's equations. For the sake of analytical tractability, analytical models are limited to simple cases. Therefore, analytical models found the basis of numerical methods, which run heavy computational tools on numerical body phantoms presented in Section III-B The FEM is the most common numerical technique to characterize the HBC channels. Numerical methods have a long simulation time and limited accuracy at low frequencies. Thus, circuit models are also developed based on an electric circuit representation of the human body's dielectric properties. A common shortcoming of these approaches is that they cannot capture the impacts of environmental effects on the channel model. As a remedy, empirical models can provide more realistic channel measurements. Nonetheless, the campaign measurements must be set very carefully as many factors affect the channel gain's true estimate, including grounding strategy, load resistance, instrumentation device specifications, and cable effects. Lastly, the variable contact impedance yields 
design uncertainty and degrades the connection reliability. In order to avoid its negative impact on the overall HBC system performance, the contact impedance elements (e.g., ESI and SSI) should be accurately modeled and dynamically matched for the sake of improved performance.

\section{COnclusions and Future Research Directions}

As an imminent extension to the IoT domain, IoB can open up significant opportunities, however, it is subject to technical challenges, security concerns, and risks. In this survey, we presented the IoB concept, specified requirements, and introduced related communications and networking standards. Then, we narrowed our scope to the characterization of channel features in-on-and-around the human body. A systematic survey of channel modeling issues is presented for various link types on NB, UWB, and HBC channels. In what follows, we bring prospective research directions to the interested readers' attention.

\section{A. Unified Channel Models}

In Section IV and Section V, it has been clearly shown that reported channel attenuation statistics significantly differs from one article to another. This is mainly due to the enormous diversity of IoB communications in terms of channel length, operating frequency, propagation modes, a myriad combination of node locations and resulting link configurations, surrounding environment, hardware specifications, simulation methods, phantom types, and measurement set up. There is a dire need to have a unified channel model database which provides key channel attributes based on frequency, distance, node locations, propagation mode, and surrounding environment. In this way, researchers and engineers can develop a more accurate system design that takes into consideration the impact of the hardware specifications (antenna matching and radiation, contact impedance of electrode, grounding effects, etc.). Although NB and UWB channel modeling issues have been studied more thoroughly, HBC channel modeling is relatively less explored. This is also the case for channel models provided by IEEE 802.15.6 [167] where HBC channels are briefly mentioned without getting into the technical details. Considering the distinct advantages offered by the HBC and the fact that it is less investigated, we see a window of opportunity for impactful contribution in this area.

\section{B. Channel Estimation Techniques}

Channel state information (CSI) is one of the most critical components to have efficient communication systems consisting of equalizers, demodulators, and decoders. The CSI can be categorized as instant and statistical. While the former describes short-term channel response to optimize the performance by adapting the transmission scheme, the latter provides a long-term description for path loss, shadowing, fading distribution, prorogation mode, etc. In practice, the statistical CSI is used along with the instant CSI to represent a communication channel. Although CSI acquisition is a challenging task for fast fading channels, this is generally not the case for IoB nodes due to the limited speed of the human mobility.

One way for channel estimation is estimating the parameters of the statistical CSI models, i.e., estimating the parameters of the combined path-loss and shadowing models or estimating the shape, scale, and skewness parameters of the underlying fading distribution [256]. Another popular approach is periodically sending and analyzing pilot or training sequences which are known by both transmitter and receiver [257]. The accuracy of pilot-aided estimation can be further improved by iterative channel estimation by using the soft information inferred from the data symbols [258]. While the parameter estimation approach is suitable to update parameters of the statistical CSI, the pilot-aided estimation is more suitable to capture time-variant SOCS metrics.

Recently, deep learning has been recognized as a powerful tool to improve overall channel estimation performance in comparison with the above traditional methods [259], [260]. Since the IoB nodes are not expected to have a high computational power, online learning approaches should be supported by offline training methods. An alternative approach would be striking a good balance between model based and data-driven learning based channel characterization. An inspiring approach is presented in [261] where authors compares model-based, learning-based, and their hybridization.

\section{Channel Aware Cross-Layer Optimization}

Cross-layer network optimization is a key enabler of running a wide variety of IoB applications on limited network resources, some of which are already in extensive use of other devices. As outlined in Section I-B. IoB applications have diverse QoS demands in terms of reliability, latency, energy efficiency, and data rate. Therefore, cross-layer optimization is a daunting challenge because of the human-body driven challenges discussed above and uncontrollable interference caused by the technologies coexisting on the same bands. The first and foremost prerequisite for an optimal cross-layer design is acquisition of accurate and timely channel estimates based on which transmission scheme can control power, manage interference, and adapt necessary coding/modulation techniques [262]. The CSI is also necessary to have an adaptive design for two critical higher layer functions: 1) Medium access control (MAC) to allow various IoB nodes to operate on the same band, and 2) Transmission control protocol to avoid congestion, sustain connectivity, and provision a reliable communication between the IoB node pairs. Body mobility and postures have already been shown to have substantial impacts on the key SOCS metrics such as delay spread, power delay profile, level crossing rate, and average fade duration. To this end, we believe it is necessary to adapt MAC layer (e.g.,packet length, frame structure, power control, channel estimation intervals, etc.) to such changes.

\section{Energy Self-Sustainability \& Network Lifetime}

IoB nodes are naturally designed as ultra-low power and low-cost devices with a limited battery capacity due to their small-form factor. However, they are still required to have 
a long operational time since either they are not reachable to charge (e.g., embedded or implanted IoB devices) or for the sake of user satisfaction (e.g., body worn devices). Therefore, energy harvesting is a key technology to design energy self-sustaining IoB devices by scavenging renewable energy sources such as thermoelectric energy from body heat [263], [264], and kinetic energy from body motions [265], [266]. It is worth noting that a proper design is necessary to strike a good balance between power consumption and system performance based on QoS demands of underlying IoB application. In order to maximize the overall network lifetime, harvested energy must be used economically by means of energy efficient cross-layer approaches [267], which still depends on accurate channel estimation. There are two main factors contributing to the power consumption in IoB nodes:

- Communication and signal processing circuitry is the most power-hungry part of the IoB devices. In addition to designing power-efficient circuit designs, energy-aware transmission strategies are of utmost importance, such as opportunistic transmission scheduling [268] and lazy packet scheduling [269]. As signal sampling and processing operations run more frequently than the signal transmission in the background, compressed sensing (i.e., sampling sparse signals at sub-Nyquist rates [270]) can significantly save energy in many IoB applications such as ECG [271], EEG [272], and EMG [273].

- Another major factor contributing to power consumption is the MAC protocol, which scales up the power consumed by communication and signal processing modules [274]. Therefore, a power-wise MAC protocol should consider energy-efficient measures such as sleeping strategy for nodes exchanging data intermittently [275]. The MAC should also minimize the number of retransmissions due to the collisions caused by interference received from nearby coexisting nodes at the same band. Therefore, carrier sense multiple access with collision avoidance (CSMA/CA) is a widely adopted technique to mitigate adverse impacts of interfering users [276]-[279]. Idle-listening and over-hearing are two other factors that further decrease the power and throughput efficiency of the MAC [280]. While the former occurs when a node listens to an idle channel to receive traffic addressed to itself, the latter happens when one node receives a packet that is intended to other nodes. A useful technique to reduce the effect of idle-listening and over-hearing is known to be low power listening, which is a preamble sampling approach [281].

Considering the fact that all these approaches counts on a precise CSI acquisition, it is obvious that channel characterization is critical to realize energy-efficient transmission and medium access schemes for energy self-sufficient IoB networks.

\section{E. Opportunities in Millimeter Wave Band}

Thanks to its abundant bandwidth availability $(3-300 \mathrm{GHz})$, millimeter Wave (mmWave) band has been considered to overcome spectrum scarcity of 5GB cellular networks, in both access and backhaul link levels. Even if exiting standards do not recognize mmWave as a component of PHY layer, it has been has recently received substantial attention to be used in BANs [111]. Despite of its high bandwidth, mmWave band suffers from high propagation loss, need for directivity, and susceptibility to blockage, which makes it more suitable for LoS communication. Therefore, it could be a good-fit for on-body and off-body links rather than the implant in-body communications [282]. Since mmWave is expected to be well integrated in future wireless networks, we believe mmWave band can open up ample opportunity for IoB applications. However, unlike the PHY techniques surveyed in this paper, there is no sufficient works on characterizing the mmWave channels on and around the human body.

\section{F. Privacy and Security}

Medical IoB applications necessitates a high level of security, confidentiality, and privacy [283]. IoB devices operating on NB and UWB channels are particularly susceptible to adversary nodes' capabilities of altering original data. Since these bands are already over-crowded by other communication devices, highly radiative and generally omni-directional nature of the NB and UWB communications inadvertently permits an eavesdropper to intercept the sensitive information. Accordingly, the confidentiality and privacy of transmitted data must be carefully guarded against the eavesdropping and overheard. Unfortunately, fulfilling these goals is a non-trivial task given the limited energy, memory, and computational power of IoB nodes.

Thanks to its low radiation footprint, $\mathrm{HBC}$ is inherently a viable alternative with its inherent physical layer security qualities. Since both forward and backward paths are confined within the body, the GC-HBC has a limited signal leakage compared to the $\mathrm{CC}-\mathrm{HBC}$ technique. To further confine signals within the body, electro-quasistatic HBC (eQs-HBC) has recently proposed as a carrier-less (broadband) transmission [284]. Thereby, eQs-HBC creates a covert communication channel where it is extremely difficult for a nearby eavesdropper to intercept critical private data. The nominal work of Das et. al. reveals that eQs leakage is detectable up to $<0.15$ $\mathrm{m}$, whereas the human body alone leaks only up to $\sim 0.01$ $\mathrm{m}$ [285]. Compared to $>5 \mathrm{~m}$ detection range for on-body NB and UWB communication, this practically suggests that the adversary needs to be in direct physical contact with or almost touching the body to obtain any information.

\section{ACKNOWLEDGEMENT}

We thank Dr. Aslihan Kartci for her valuable discussions on Section $\mathrm{V}-\mathrm{C}$ and help in preparing Table VIII. Table IX, and Figure 5. We also thank Ms. Kenan S. Sindi for her participation in preparing Table III and Table IV

\section{REFERENCES}

[1] L. Atzori, A. Iera, and G. Morabito, "The internet of things: A survey," Computer networks, vol. 54, no. 15, pp. 2787-2805, 2010.

[2] M. Agiwal, A. Roy, and N. Saxena, "Next generation $5 \mathrm{~g}$ wireless networks: A comprehensive survey," IEEE Commun. Surveys Tuts. vol. 18 , no. 3 , pp. 1617-1655, 2016. 
[3] N. Saeed, A. Elzanaty, H. Almorad, H. Dahrouj, T. Y. Al-Naffouri, and M.-S. Alouini, "Cubesat communications: Recent advances and future challenges," IEEE Commun. Surveys Tuts., 2020.

[4] M. J. O’Mahony, C. Politi, D. Klonidis, R. Nejabati, and D. Simeonidou, "Future optical networks," Journal of Lightwave Technology, vol. 24, no. 12, pp. 4684-4696, 2006.

[5] I. F. Akyildiz, W. Su, Y. Sankarasubramaniam, and E. Cayirci, "Wireless sensor networks: a survey," Computer networks, vol. 38, no. 4, pp. 393-422, 2002.

[6] M. Chen, S. Gonzalez, A. Vasilakos, H. Cao, and V. C. M. Leung, "Body area networks: A survey," Mobile Networks and Applications, vol. 16, no. 2, pp. 171-193, 2011.

[7] A. Celik and A. E. Kamal, "Multi-objective clustering optimization for multi-channel cooperative spectrum sensing in heterogeneous green crns," IEEE Trans. Cognitive Commun. Netw., vol. 2, no. 2, pp. 150$161,2016$.

[8] A. Celik, A. Alsharoa, and A. E. Kamal, "Hybrid energy harvestingbased cooperative spectrum sensing and access in heterogeneous cognitive radio networks," IEEE Trans. Cognitive Commun. Netw., vol. 3 , no. 1, pp. 37-48, 2017.

[9] A. Celik and A. E. Kamal, "Green cooperative spectrum sensing and scheduling in heterogeneous cognitive radio networks," IEEE Trans. Cognitive Commun. Netw., vol. 2, no. 3, pp. 238-248, 2016.

[10] "Wireless LAN medium access control (MAC) and physical layer (PHY) specifications," IEEE Std 802.11-2016, pp. 1-3534, Dec. 2016

[11] D. Tsonev, S. Videv, and H. Haas, "Light fidelity (li-fi): towards alloptical networking," in Broadband Access Communication Technologies VIII, vol. 9007. International Society for Optics and Photonics, 2014, p. 900702.

[12] "Specification of the bluetooth system version 5.1," Bluetooth SIG, pp. 1-2985, Jan. 2019.

[13] "Zigbee specifications 05-3474-21," ZigBee Alliance, pp. 1-542, Aug. 2015 .

[14] O. Georgiou and U. Raza, "Low power wide area network analysis: Can lora scale?" IEEE Wireless Commun. Lett., vol. 6, no. 2, pp. 162 165, 2017.

[15] I. F. Akyildiz and A. Kak, "The internet of space things/cubesats: A ubiquitous cyber-physical system for the connected world," Computer Networks, vol. 150, pp. 134-149, 2019.

[16] N. Saeed, M.-S. Alouini, and T. Y. Al-Naffouri, "Toward the internet of underground things: A systematic survey," IEEE Commun. Surveys Tuts., vol. 21, no. 4, pp. 3443-3466, 2019.

[17] A. Celik, N. Saeed, B. Shihada, T. Y. Al-Naffouri, and M.-S. Alouini, "A software-defined opto-acoustic network architecture for internet of underwater things," IEEE Commun. Mag., vol. 58, no. 4, pp. 88-94, 2020.

[18] S. Jeschke, C. Brecher, T. Meisen, D. Özdemir, and T. Eschert, "Industrial internet of things and cyber manufacturing systems," in Industrial internet of things. Springer, 2017, pp. 3-19.

[19] A. Ometov, S. V. Bezzateev, J. Kannisto, J. Harju, S. Andreev, and Y. Koucheryavy, "Facilitating the delegation of use for private devices in the era of the internet of wearable things," IEEE Internet Things J., vol. 4, no. 4, pp. 843-854, 2016.

[20] P. Fraga-Lamas, T. M. Fernández-Caramés, M. Suárez-Albela, L. Castedo, and M. González-López, "A review on internet of things for defense and public safety," Sensors, vol. 16, no. 10, p. 1644, 2016.

[21] C. A. da Costa, C. F. Pasluosta, B. Eskofier, D. B. da Silva, and R. da Rosa Righi, "Internet of health things: Toward intelligent vital signs monitoring in hospital wards," Artificial intelligence in medicine, vol. 89, pp. 61-69, 2018.

[22] B. Latré, B. Braem, I. Moerman, C. Blondia, and P. Demeester, "A survey on wireless body area networks," Wireless Networks, vol. 17, no. 1 , pp. 1-18, 2011.

[23] G. Schalk, D. J. McFarland, T. Hinterberger, N. Birbaumer, and J. R. Wolpaw, "Bci2000: a general-purpose brain-computer interface (bci) system," IEEE Trans. Biomed. Eng., vol. 51, no. 6, pp. 1034-1043, 2004.

[24] Wikipedia contributors, "Microchip implant (human) - Wikipedia, the free encyclopedia," 2020, [Online; accessed 5-July2020]. [Online]. Available: https://en.wikipedia.org/w/index.php?title= Microchip_implant_(human)\&oldid=965261230

[25] A. Milenković, C. Otto, and E. Jovanov, "Wireless sensor networks for personal health monitoring: Issues and an implementation," Computer communications, vol. 29, no. 13-14, pp. 2521-2533, 2006.

[26] L. Lorenzoni, A. Marino, D. Morgan, and C. James, "Health spending projections to 2030," no. 110, 2019. [Online]. Available: https://www.oecd-ilibrary.org/content/paper/5667f23d-en
[27] "Novel coronavirus 2019 situation report-209,", The World Health Organization, Tech. Rep., Jul. 2020. [Online]. Available: https://www. who.int/emergencies/diseases/novel-coronavirus-2019/situation-reports

[28] "Pandemic, recession: The global economy in crisis,", The World Bank, Tech. Rep., Jun. 2020. [Online]. Available: https://www. worldbank.org/en/publication/global-economic-prospects

[29] P. S. Hall, Y. Hao, Y. I. Nechayev, A. Alomainy, C. C. Constantinou, C. Parini, M. R. Kamarudin, T. Z. Salim, D. T. M. Hee, R. Dubrovka, A. S. Owadally, W. Song, A. Serra, P. Nepa, M. Gallo, and M. Bozzetti, "Antennas and propagation for on-body communication systems," IEEE Antennas and Propagation Magazine, vol. 49, no. 3, pp. 41-58, 2007.

[30] H. Baldus, S. Corroy, A. Fazzi, K. Klabunde, and T. Schenk, "Humancentric connectivity enabled by body-coupled communications," IEEE Commun. Mag., vol. 47, no. 6, pp. 172-178, 2009.

[31] H. Cao, V. Leung, C. Chow, and H. Chan, "Enabling technologies for wireless body area networks: A survey and outlook," IEEE Commun. Mag., vol. 47, no. 12, pp. 84-93, Dec. 2009.

[32] S. Ullah, H. Higgins, B. Braem, B. Latre, C. Blondia, I. Moerman, S. Saleem, Z. Rahman, and K. S. Kwak, "A comprehensive survey of wireless body area networks," Journal of Medical Systems, vol. 36, no. 3, pp. 1065-1094, 2012.

[33] R. Chavez-Santiago, K. Sayrafian-Pour, A. Khaleghi, K. Takizawa, J. Wang, I. Balasingham, and H. Li, "Propagation models for ieee 802.15.6 standardization of implant communication in body area networks," IEEE Commun. Mag., vol. 51, no. 8, pp. 80-87, Aug. 2013.

[34] D. B. Smith, D. Miniutti, T. A. Lamahewa, and L. W. Hanlen, "Propagation models for body-area networks: A survey and new outlook," IEEE Antenna Propag. Mag., vol. 55, no. 5, pp. 97-117, Oct. 2013.

[35] M. Seyedi, B. Kibret, D. T. H. Lai, and M. Faulkner, "A survey on intrabody communications for body area network applications," IEEE Trans. Biomed. Eng., vol. 60, no. 8, pp. 2067-2079, Aug. 2013.

[36] S. Movassaghi, M. Abolhasan, J. Lipman, D. Smith, and A. Jamalipour, "Wireless body area networks: A survey," IEEE Commun. Surveys Tuts., vol. 16, no. 3, pp. 1658-1686, Third 2014.

[37] R. Cavallari, F. Martelli, R. Rosini, C. Buratti, and R. Verdone, "A survey on wireless body area networks: Technologies and design challenges," IEEE Commun. Surveys Tuts., vol. 16, no. 3, pp. 16351657, Third 2014.

[38] J. F. Zhao, X. M. Chen, B. D. Liang, and Q. X. Chen, "A review on human body communication: signal propagation model, communication performance, and experimental issues," Wireless Communications and Mobile Computing, vol. 2017, 2017.

[39] W. J. Tomlinson, S. Banou, C. Yu, M. Stojanovic, and K. R. Chowdhury, "Comprehensive survey of galvanic coupling and alternative intrabody communication technologies," IEEE Commun. Surveys Tuts. vol. 21, no. 2, pp. 1145-1164, 2018.

[40] "Wireless LAN medium access control (MAC) and physical layer (PHY) specifications for wireless personal area networks (WPAN)," IEEE Std 802.15.1-2005, pp. 1-700, Jun. 2005.

[41] "IEEE standard for low-rate wireless networks," IEEE Std 802.15.42015, pp. 1-709, Apr. 2016

[42] M. Hernandez and R. Kohno, "Coexistence of uwb-bans with other wireless systems," in 2009 International Symposium on Intelligent Signal Processing and Communication Systems (ISPACS), Jan. 2009, pp. 135-137.

[43] F. Martelli and R. Verdone, "Coexistence issues for wireless body area networks at $2.45 \mathrm{GHz}$," in 18th European Wireless Conference, Apr. 2012, pp. 1-6.

[44] M. Hernandez and R. Miura, "Coexistence of ieee std 802.15.6tm2012 uwb-phy with other uwb systems," in 2012 IEEE International Conference on Ultra-Wideband, Sep. 2012, pp. 46-50.

[45] I. Guideline, "Guidelines for limiting exposure to time-varying electric, magnetic, and electromagnetic fields (up to $300 \mathrm{ghz}$ )," Health phys, vol. 74, no. 4, pp. 494-522, 1998.

[46] "FCC encyclopedia - radio frequency safety," Scientific Committee on Emerging and Newly Identified Health Risks (SCENIHR), Tech. Rep., Jan. 2015. [Online]. Available: https://ec.europa.eu/health/scientific_committees/consultations/ public_consultations/scenihr_consultation_19_en

[47] "Potential health effects of exposure to electromagnetic fields (EMF)," Federal Commissions on Communications, Tech. Rep. [Online]. Available: https://www.fcc.gov/general/radio-frequency-safety-0

[48] V. S. Mallela, V. Ilankumaran, and N. S. Rao, "Trends in cardiac pacemaker batteries," Indian pacing and electrophysiology journal, vol. 4, no. 4, p. 201, 2004.

[49] A. M. Hussain and M. M. Hussain, "Cmos-technology-enabled flexible and stretchable electronics for internet of everything 
applications," Advanced Materials, vol. 28, no. 22, pp. 4219-4249, 2016. [Online]. Available: https://onlinelibrary.wiley.com/doi/abs/10. 1002/adma.201504236

[50] S. Cheng, A. Rydberg, K. Hjort, and Z. Wu, "Liquid metal stretchable unbalanced loop antenna," Applied Physics Letters, vol. 94, no. 14, p. 144103, 2009.

[51] M. Kubo, X. Li, C. Kim, M. Hashimoto, B. J. Wiley, D. Ham, and G. M. Whitesides, "Stretchable microfluidic radiofrequency antennas," Advanced materials, vol. 22, no. 25, pp. 2749-2752, 2010.

[52] L. H. Sverdrup Jr, N. F. Dessel, and A. Pelkus, "Thin film flexible solar cell," Apr. 15 2003, uS Patent 6,548,751.

[53] D. W. Carroll, "Wearable personal computer system having flexible battery forming casing of the system," Nov. 5 1996, uS Patent 5,572,401.

[54] A. Nag, S. C. Mukhopadhyay, and J. Kosel, "Wearable flexible sensors: A review," IEEE Sensors Journal, vol. 17, no. 13, pp. 3949-3960, 2017.

[55] M. Gao, L. Li, and Y. Song, "Inkjet printing wearable electronic devices," Journal of Materials Chemistry C, vol. 5, no. 12, pp. 29712993, 2017.

[56] D. Kim and J. Moon, "Highly conductive ink jet printed films of nanosilver particles for printable electronics," Electrochemical and Solid-State Letters, vol. 8, no. 11, pp. J30-J33, 2005.

[57] C. C. Poon, Y.-T. Zhang, and S.-D. Bao, "A novel biometrics method to secure wireless body area sensor networks for telemedicine and $\mathrm{m}$ health," IEEE Commun. Mag., vol. 44, no. 4, pp. 73-81, 2006.

[58] S. Cherukuri, K. K. Venkatasubramanian, and S. K. Gupta, "BIOSEC: A biometric based approach for securing communication in wireless networks of biosensors implanted in the human body," in 2003 International Conference on Parallel Processing Workshops, 2003. Proceedings. IEEE, 2003, pp. 432-439.

[59] "Ipv6 over low power wpan (6lowpan)," Internet Engineering Task Force (IETF). [Online]. Available: https://datatracker.ietf.org/wg/ 6lowpan/documents/

[60] "Ieee standard for wireless body area networks," IEEE Std 802.15.62012, pp. 1-271, Feb. 2012.

[61] E. M. Staderini, "Uwb radars in medicine," IEEE aerospace and electronic systems magazine, vol. 17, no. 1, pp. 13-18, 2002.

[62] J. Wang and Q. Wang, Body area communications: channel modeling, communication systems, and EMC. John Wiley \& Sons, 2012.

[63] H. Kanai, M. Haeno, and K. Sakamoto, "Electrical measurement of fluid distribution in legs and arms," in Medical Progress through technology. Springer, 1987, pp. 159-170.

[64] C. Li, M.-R. Tofighi, D. Schreurs, and T.-S. J. Horng, Principles and Applications of RF/microwave in Healthcare and Biosensing. Academic Press, 2016.

[65] J. D. Bronzino and D. R. Peterson, Biomedical engineering fundamentals. CRC press, 2014

[66] H. P. Schwan, "Electrical properties of tissue and cell suspensions," in Advances in biological and medical physics. Elsevier, 1957, vol. 5 , pp. 147-209.

[67] K. S. Cole and R. H. Cole, "Dispersion and absorption in dielectrics i. alternating current characteristics," The Journal of chemical physics, vol. 9 , no. 4, pp. 341-351, 1941.

[68] S. Gabriel, R. W. Lau, and C. Gabriel, "The dielectric properties of biological tissues: II. measurements in the frequency range $10 \mathrm{hz}$ to $20 \mathrm{GHz}, "$ Physics in Medicine and Biology, vol. 41, no. 11, pp. 2251-2269, nov 1996. [Online]. Available: https: //doi.org/10.1088\%2F0031-9155\%2F41\%2F11\%2F002

[69] Jianqing Wang, O. Fujiwara, and S. Watanabe, "Approximation of aging effect on dielectric tissue properties for sar assessment of mobile telephones," IEEE Trans. Electromagn. Compat., vol. 48, no. 2, pp. 408-413, 2006

[70] J. B. Keller, "Geometrical theory of diffraction," Josa, vol. 52, no. 2, pp. 116-130, 1962.

[71] B. Mohammed, A. Abbosh, B. Henin, and P. Sharpe, "Head phantom for testing microwave systems for head imaging," 2012 Cairo International Biomedical Engineering Conference (CIBEC), 2012

[72] Z. N. Chen, G. C. Liu, and T. S. P. See, "Transmission of rf signals between mics loop antennas in free space and implanted in the human head," IEEE Trans. Antennas Propag., vol. 57, no. 6, p. 1850-1854, 2009.

[73] K. S. Moon, H. D. Choi, A. K. Lee, K. Y. Cho, H. G. Yoon, and K. S. Suh, "Dielectric properties of epoxy-dielectrics-carbon black composite for phantom materials at radio frequencies," Journal of Applied Polymer Science, vol. 77, no. 6, p. 1294-1302, 2000.

[74] S. Mochizuki, H. Wakayanagi, T. Hamada, S. Watanabe, M. Taki, Y. Yamanaka, and H. Shirai, "Effects of ear shape and head size on sim- ulated head exposure to a cellular phone," IEEE Trans. Electromagn. Compat., vol. 49, no. 3, p. 512-518, 2007.

[75] V. Hombach, K. Meier, M. Burkhardt, E. Kuhn, and N. Kuster, "The dependence of em energy absorption upon human head modeling at 900 mhz," IEEE Trans. Microw. Theory Techn., vol. 44, no. 10, p. $1865-1873,1996$.

[76] K. Ogawa and T. Matsuyoshi, "An analysis of the performance of a handset diversity antenna influenced by head, hand, and shoulder effects at $900 \mathrm{mhz}$.i. effective gain characteristics," IEEE Trans. Veh. Technol., vol. 50, no. 3, p. 830-844, 2001.

[77] F. Zhang, X. Liu, S. A. Hackworth, R. J. Sclabassi, and M. Sun, "In vitro and in vivo studies on wireless powering of medical sensors and implantable devices," 2009 IEEE/NIH Life Science Systems and Applications Workshop, 2009.

[78] S.-I. Watanabe, H. Taki, T. Nojima, and O. Fujiwara, "Characteristics of the sar distributions in a head exposed to electromagnetic fields radiated by a hand-held portable radio," IEEE Trans. Microw. Theory Techn., vol. 44, no. 10, p. 1874-1883, 1996

[79] T. Kobayashi, T. Nojima, K. Yamada, and S. Uebayashi, "Dry phantom composed of ceramics and its application to sar estimation," IEEE Trans. Microw. Theory Techn., vol. 41, no. 1, p. 136-140, 1993.

[80] H. Tamura, Y. Ishikawa, T. Kobayashi, and T. Nojima, "A dry phantom material composed of ceramic and graphite powder," IEEE Trans. Electromagn. Compat., vol. 39, no. 2, p. 132-137, 1997.

[81] V. Monebhurrun, "Conservativeness of the sam phantom for the sar evaluation in the child's head,", IEEE Trans. Magn., vol. 46, no. 8, p. $3477-3480$.

[82] O. Saraereh et al., "Simulation and experimental sar and efficiency study for a dual-band pifa handset antenna (gsm $900 /$ des 1800) at varied distances from a phantom head," Technical Seminar on Antenna Measurements and SAR (AMS 2004), 2004.

[83] C. K. Looi and Z. N. Chen, "Design of a human-head-equivalent phantom for ism 2.4-ghz applications," Microw. Opt. Technol. Lett, vol. 47 , no. 2 , p. $163-166$, Oct. 2005

[84] C. K. Looi, T. See, and Z. N. Chen, "Study of human head effects on the planar inverted-f antenna," IWAT 2005. IEEE International Workshop on Antenna Technology: Small Antennas and Novel Metamaterials, 2005.

[85] I. Youngs, G. Fixter, A. Treen, and S. Holden, "Design of solid broadband human tissue simulant materials," IEE Proceedings - Science, Measurement and Technology, vol. 149, no. 6, p. 323-328, Jan 2002.

[86] K. Ito, Y. Okano, A. Hase, and I. Ida, "A tissue-equivalent solid phantom for estimation of interaction between human head and handset antenna," 1998 IEEE-APS Conference on Antennas and Propagation for Wireless Communications (Cat. No.98EX184).

[87] A. T. Mobashsher, A. M. Abbosh, and Y. Wang, "Microwave system to detect traumatic brain injuries using compact unidirectional antenna and wideband transceiver with verification on realistic head phantom," IEEE Trans. Microw. Theory Techn., vol. 62, no. 9, p. 1826-1836, 2014.

[88] A. T. Mobashsher and A. M. Abbosh, "Three-dimensional human head phantom with realistic electrical properties and anatomy," IEEE Antennas and Wireless Propagation Letters, vol. 13, p. 1401-1404, 2014.

[89] B. J. Mohammed, A. M. Abbosh, S. Mustafa, and D. Ireland, "Microwave system for head imaging," IEEE Trans. Instrum. Meas. vol. 63, no. 1, p. 117-123, 2014.

[90] B. J. Mohammed and A. M. Abbosh, "Realistic head phantom to test microwave systems for brain imaging," Microwave and Optical Technology Letters, vol. 56, no. 4, p. 979-982, 2014.

[91] S. Mustafa, B. Mohammed, and A. Abbosh, "Novel preprocessing techniques for accurate microwave imaging of human brain," IEEE Antennas and Wireless Propagation Letters, vol. 12, p. 460-463, 2013

[92] A. T. Mobashsher, B. J. Mohammed, S. Mustafa, and A. Abbosh, "Ultra wideband antenna for portable brain stroke diagnostic system," 2013 IEEE MTT-S International Microwave Workshop Series on RF and Wireless Technologies for Biomedical and Healthcare Applications (IMWS-BIO), 2013.

[93] H. N. Schwerdt, J. Chae, and F. A. Miranda, "Wireless performance of a fully passive neurorecording microsystem embedded in dispersive human head phantom," Proceedings of the 2012 IEEE International Symposium on Antennas and Propagation, 2012.

[94] H. N. Schwerdt, F. A. Miranda, and J. Chae, "A fully passive wireless backscattering neurorecording microsystem embedded in dispersive human-head phantom medium," IEEE Electron Device Letters, vol. 33 , no. 6, p. 908-910, 2012. 
[95] R. Ishido, "A study in the solid phantom for 3-6 ghz and evaluation of sar distribution based on the thermographic method," EMC'04, Sendai, Japan, June, 2004

[96] G. Conway, W. Scanlon, C. Orlenius, and C. Walker, "In situ measurement of uhf wearable antenna radiation efficiency using a reverberation chamber," IEEE Antennas and Wireless Propagation Letters, vol. 7, p. 271-274, 2008.

[97] G. Conway, W. Scanlon, and S. Cotton, "The performance of on-body wearable antennas in a repeatable multipath environment," 2008 IEEE Antennas and Propagation Society International Symposium, 2008.

[98] G. Gajda, M. A. Stuchly, and S. S. Stuchly, "Mapping of the near- field pattern in simulated biological tissues,", Electron Lett, vol. 15, no. 4, Feb. 1979.

[99] A. Cheung and D. Koopman, "Experimental development of simulated biomaterials for dosimetry studies of hazardous microwave radiation (short papers)," IEEE Trans. Microw. Theory Techn., vol. 24, no. 10, p. 669-673, 1976.

[100] T. Yamamoto, K. Sano, K. Koshiji, X. Chen, S. Yang, M. Abe, and A. Fukuda, "Development of electromagnetic phantom at lowfrequency band," 2013 35th Annual International Conference of the IEEE Engineering in Medicine and Biology Society (EMBC), 2013.

[101] H. Kato and T. Ishida, "Development of an agar phantom adaptable for simulation of various tissues in the range 5-40 mhz. (hyperthermia treatment of cancer)," Physics in Medicine and Biology, vol. 32, no. 2, p. 221-226, Jan. 1987

[102] G. Hartsgrove, A. Kraszewski, and A. Surowiec, "Simulated biological materials for electromagnetic radiation absorption studies," Bioelectromagnetics, vol. 8, no. 1, p. 29-36, 1987.

[103] V. Vigneras and F. Bonnaudin, "Biological tissues equivalent liquids in the frequency range 900-3000 mhz," Proc. XXVIIIth URSI General Assembly, p. 1-4, 2005.

[104] T. Takimoto, T. Onishi, K. Saito, M. Takahashi, S. Uebayashi, and K. Ito, "Characteristics of biological tissue equivalent phantoms applied to uwb communications," Electronics and Communications in Japan (Part I: Communications), vol. 90, no. 5, p. 48-55, 2007.

[105] Y. Yuan, C. Wyatt, P. Maccarini, P. Stauffer, O. Craciunescu, J. Macfall, M. Dewhirst, and S. K. Das, "A heterogeneous human tissue mimicking phantom for rf heating and mri thermal monitoring verification," Physics in Medicine and Biology, vol. 57, no. 7, p. 2021-2037, 2012.

[106] C. Gabriel, "Tissue equivalent material for hand phantoms," Physics in Medicine and Biology, vol. 52, no. 14, p. 4205-4210, 2007.

[107] N. Chahat, M. Zhadobov, and R. Sauleau, "Broadband tissue-equivalent phantom for ban applications at millimeter waves," IEEE Trans. Microw. Theory Techn., vol. 60, no. 7, pp. 2259-2266, 2012.

[108] N. Chahat, C. Leduc, M. Zhadobov, and R. Sauleau, "Antennas and interaction with the body for body-centric wireless communications at millimeter-waves," in 2013 7th European Conference on Antennas and Propagation (EuCAP). IEEE, 2013, pp. 772-775.

[109] L. Zhang, Z. Wang, and J. L. Volakis, "Textile antennas and sensors for body-worn applications," IEEE Antennas and Wireless Propagation Letters, vol. 11, p. 1690-1693, 2012.

[110] G. Hartsgrove, A. Kraszewski, and A. Surowiec, "Simulated biological materials for electromagnetic radiation absorption studies," Bioelectromagnetics: Journal of the Bioelectromagnetics Society, The Society for Physical Regulation in Biology and Medicine, The European Bioelectromagnetics Association, vol. 8, no. 1, pp. 29-36, 1987.

[111] A. Pellegrini, A. Brizzi, L. Zhang, K. Ali, Y. Hao, X. Wu, C. Constantinou, Y. Nechayev, P. Hall, N. Chahat et al., "Antennas and propagation for body-centric wireless communications at millimeterwave frequencies: A review [wireless corner]," IEEE Antennas and Propagation Magazine, vol. 55, no. 4, pp. 262-287, 2013.

[112] T. Cuyckens, "Influence of the human body on the behaviour of monopole and patch antennas," Ph.D. dissertation, $\mathrm{Ph}$. D. dissertation, University Gent.[online]. Available: http://lib. ugent ..., 2010.

[113] P. S. Hall and Y. Hao, Antennas and propagation for body-centric wireless communications. Artech house, 2012 .

[114] A. E. Khorshid, I. N. Alquaydheb, A. M. Eltawil, and F. J. Kurdahi, "Physical multi-layer phantoms for intra-body communications," IEEE Access, vol. 6, pp. 42 812-42821, 2018.

[115] — - "Ibcfap: Intra-body communications five-layers arm phantom model," IEEE Access, vol. 7, pp. 93701-93710, 2019.

[116] A. W. Guy, "Analyses of electromagnetic fields induced in biological tissues by thermographic studies on equivalent phantom models," IEEE Trans. Microw. Theory Techn., vol. 19, no. 2, pp. 205-214, 1971.

[117] C.-K. Chou, G.-W. Chen, A. W. Guy, and K. H. Luk, "Formulas for preparing phantom muscle tissue at various radiofrequencies," Bioelectromagnetics: Journal of the Bioelectromagnetics Society, The
Society for Physical Regulation in Biology and Medicine, The European Bioelectromagnetics Association, vol. 5, no. 4, pp. 435-441, 1984.

[118] A. T. Mobashsher and A. Abbosh, "Three-dimensional human head phantom with realistic electrical properties and anatomy," IEEE Antennas and Wireless Propagation Letters, vol. 13, pp. 1401-1404, 2014.

[119] N. Chahat, M. Zhadobov, and R. Sauleau, "Broadband tissue-equivalent phantom for ban applications at millimeter waves," IEEE Trans. Microw. Theory Techn., vol. 60, no. 7, pp. 2259-2266, 2012.

[120] N. Chahat, M. Zhadobov, S. Alekseev, and R. Sauleau, "Human skinequivalent phantom for on-body antenna measurements in $60 \mathrm{ghz}$ band," Electronics letters, vol. 48, no. 2, pp. 67-68, 2012.

[121] H. Tamura, Y. Ishikawa, T. Kobayashi, and T. Nojima, "A dry phantom material composed of ceramic and graphite powder," IEEE Trans. Electromagn. Compat., vol. 39, no. 2, pp. 132-137, 1997.

[122] M.-C. Gosselin et al., "Development of a new generation of high-resolution anatomical models for medica 1 device evaluation: the virtual population 3.0," Physics in Medicine and Biology, vol. 59, no. 18, pp. 5287-5303, aug 2014. [Online]. Available: https://doi.org/10.1088\%2F0031-9155\%2F59\%2F18\%2F5287

[123] M. Mazzurana, L. Sandrini, A. Vaccari, C. Malacarne, L. Cristoforetti, and R. Pontalti, "A semi-automatic method for developing an anthropomorphic numerical model of dielectric anatomy by MRI," Physics in Medicine and Biology, vol. 48, no. 19, pp. 3157-3170, sep 2003.

[124] H. N. Kritikos and H. P. Schwan, "Hot spots generated in conducting spheres by electromagnetic waves and biological implications," IEEE Trans. Biomed. Eng., vol. BME-19, no. 1, pp. 53-58, 1972.

[125] A. R. Shapiro, R. F. Lutomirski, and H. T. Yura, "Induced fields and heating within a cranial structure irradiated by an electromagnetic plane wave," IEEE Trans. Microw. Theory Techn., vol. 19, no. 2, pp. 187-196, 1971

[126] W. T. Joines and R. J. Spiegel, "Resonance absorption of microwaves by the human skull," IEEE Trans. Biomed. Eng., no. 1, pp. 46-48, 1974.

[127] C. M. Weil, "Absorption characteristics of multilayered sphere models exposed to uhf/microwave radiation," IEEE Trans. Biomed. Eng., no. 6, pp. 468-476, 1975.

[128] H. Massoudi, C. H. Durney, P. W. Barber, and M. F. Iskander, "Electromagnetic absorption in multilayered cylindrical models of man," IEEE Trans. Microw. Theory Techn., vol. 27, no. 10, pp. 825-830, 1979.

[129] S. Nishizawa, "0. hashimoto," effective shielding analysis for three layered human model,"," IEEE Trans. Microwave Theory Tech, vol. 47, no. 3, pp. 277-283, 1999.

[130] C. WG3, "Proposal for numerical canonical models in mobile communications," 1994.

[131] A. Christ et al., "The virtual family-development of surface-based anatomical models of two adults and two children for dosimetric simulations," Physics in Medicine \& Biology, vol. 55, no. 2, p. N23, 2009

[132] N. Petoussi-Henss, M. Zankl, U. Fill, and D. Regulla, "The gsf family of voxel phantoms," Physics in Medicine \& Biology, vol. 47, no. 1 , p. 89,2001

[133] C. Waldby, The visible human project: Informatic bodies and posthuman medicine. Psychology Press, 2000.

[134] T. Nagaoka, S. Watanabe, K. Sakurai, E. Kunieda, S. Watanabe, M. Taki, and Y. Yamanaka, "Development of realistic high-resolution whole-body voxel models of japanese adult males and females of average height and weight, and application of models to radiofrequency electromagnetic-field dosimetry," Physics in Medicine \& Biology, vol. 49, no. 1, p. 1, 2003.

[135] T. Wu, L. Tan, Q. Shao, C. Zhang, C. Zhao, Y. Li, E. Conil, A. Hadjem, J. Wiart, B. Lu et al., "Chinese adult anatomical models and the application in evaluation of rf exposures," Physics in Medicine \& Biology, vol. 56, no. 7, p. 2075, 2011.

[136] M. Ghaddar, L. Talbi, T. A. Denidni, and A. Sebak, "A conducting cylinder for modeling human body presence in indoor propagation channel," IEEE Trans. Antennas Propag., vol. 55, no. 11, pp. 30993103, 2007.

[137] M. Ghaddar, L. Talbi, T. Denidni, and A. Charbonneau, "Modeling human body effects for indoor radio channel using utd," in Canadian Conference on Electrical and Computer Engineering 2004 (IEEE Cat. No. 04CH37513), vol. 3. IEEE, 2004, pp. 1357-1360.

[138] D. S. Ahluwalia, "Uniform asymptotic theory of diffraction by the edge of a three-dimensional body," SIAM Journal on Applied Mathematics, vol. 18, no. 2, pp. 287-301, 1970.

[139] Y. Zhao, Y. Hao, A. Alomainy, and C. Parini, "Uwb on-body radio channel modeling using ray theory and subband fdtd method," IEEE Trans. Microw. Theory Techn., vol. 54, no. 4, pp. 1827-1835, 2006. 
[140] S. Collonge, G. Zaharia, and G. E. Zein, "Influence of the human activity on wide-band characteristics of the $60 \mathrm{ghz}$ indoor radio channel," IEEE Trans. Wireless Commun., vol. 3, no. 6, pp. 2396-2406, 2004.

[141] G. Koutitas, "Multiple human effects in body area networks," IEEE Antennas and Wireless Propagation Letters, vol. 9, pp. 938-941, 2010.

[142] R. F. Harrington, Field computation by moment methods. Wiley-IEEE Press, 1993.

[143] K. Ito, I. Ida, and M.-S. Wu, "Body effect on characteristics of small loop antenna in pager systems," in IEEE Antennas and Propagation Society International Symposium 1992 Digest. IEEE, 1992, pp. 10811084

[144] H.-R. Chuang and W.-T. Chen, "Computer simulation of the humanbody effects on a circular-loop-wire antenna for radio-pager communications at 152, 280, and $400 \mathrm{mhz}$," IEEE Trans. Veh. Technol., vol. 46, no. 3, pp. 544-559, 1997

[145] D. Psychoudakis, G. Lee, C. Chen, and J. Volakis, "Body-worn diversity antennas for squad area networks (san)," in URSI General Assembly, 2008, pp. 1-4.

[146] W.-T. Chen and H.-R. Chuang, "Numerical computation of human interaction with arbitrarily oriented superquadric loop antennas in personal communications," IEEE Trans. Antennas Propag., vol. 46, no. 6, pp. 821-828, 1998.

[147] _ "Numerical computation of the em coupling between a circular loop antenna and a full-scale human-body model," IEEE Trans. Microw. Theory Techn., vol. 46, no. 10, pp. 1516-1520, 1998.

[148] I. Farmaga, P. Shmigelskyi, P. Spiewak, and L. Ciupinski, "Evaluation of computational complexity of finite element analysis," in 2011 11th International Conference The Experience of Designing and Application of CAD Systems in Microelectronics (CADSM). IEEE, 2011, pp. 213214.

[149] R. Xu, H. Zhu, and J. Yuan, "Circuit-coupled fem analysis of the electric-field type intra-body communication channel," in 2009 IEEE Biomedical Circuits and Systems Conference, 2009, pp. 221-224.

[150] R. Xu, H. Zhu, and J. Yuan, "Electric-field intrabody communication channel modeling with finite-element method," IEEE Trans. Biomed. Eng., vol. 58, no. 3, pp. 705-712, 2011.

[151] Y. Song, K. Zhang, Q. Hao, L. Hu, J. Wang, and F. Shang, "A finiteelement simulation of galvanic coupling intra-body communication based on the whole human body," Sensors, vol. 12, no. 10, pp. $13567-$ $13582,2012$.

[152] M. A. Callejón, J. Reina-Tosina, D. Naranjo-Hernández, and L. M. Roa, "Galvanic coupling transmission in intrabody communication: A finite element approach," IEEE Trans. Biomed. Eng., vol. 61, no. 3, pp. 775-783, 2014.

[153] J. Mao, H. Yang, and B. Zhao, "An investigation on ground electrodes of capacitive coupling human body communication," IEEE Trans. Biomed. Circuits Syst., vol. 11, no. 4, pp. 910-919, 2017.

[154] M. A. Callejón, P. del Campo, J. Reina-Tosina, and L. M. Roa, "A parametric computational analysis into galvanic coupling intrabody communication," IEEE Journal of Biomedical and Health Informatics, vol. 22, no. 4, pp. 1087-1096, 2018.

[155] K. Yee, "Numerical solution of initial boundary value problems involving maxwell's equations in isotropic media," IEEE Trans. Antennas Propag., vol. 14, no. 3, pp. 302-307, 1966.

[156] A. Taflove and S. C. Hagness, Computational electrodynamics: the finite-difference time-domain method. Artech house, 2005.

[157] J.-Y. Chen and O. P. Gandhi, "Currents induced in an anatomically based model of a human for exposure to vertically polarized electromagnetic pulses," IEEE Trans. Microw. Theory Techn., vol. 39, no. 1, pp. 31-39, 1991.

[158] G. Lazzi and O. P. Gandhi, "A mixed fdtd-integral equation approach for on-site safety assessment in complex electromagnetic environments," IEEE Trans. Antennas Propag., vol. 48, no. 12, pp. 1830-1836, 2000.

[159] M. A. Jensen and Y. Rahmat-Samii, "Em interaction of handset antennas and a human in personal communications," Proceedings of the IEEE, vol. 83, no. 1, pp. 7-17, 1995.

[160] C. M. Furse, J.-Y. Chen, and O. P. Gandhi, "The use of the frequencydependent finite-difference time-domain method for induced current and sar calculations for a heterogeneous model of the human body,' IEEE Trans. Electromagn. Compat., vol. 36, no. 2, pp. 128-133, 1994.

[161] P. Dimbylow and S. Mann, "Sar calculations in an anatomically realistic model of the head for mobile communication transceivers at $900 \mathrm{mhz}$ and 1.8 ghz," Physics in Medicine \& Biology, vol. 39, no. 10, p. 1537, 1994.
[162] K. Fujii, K. Ito, and S. Tajima, "A study on the receiving signal level in relation with the location of electrodes for wearable devices using human body as a transmission channel," in IEEE Antennas and Propagation Society International Symposium. Digest. Held in conjunction with: USNC/CNC/URSI North American Radio Sci. Meeting (Cat. No. 03CH37450), vol. 3. IEEE, 2003, pp. 1071-1074.

[163] K. Fujii and K. Ito, "Evaluation of the received signal level in relation to the size and carrier frequencies of the wearable device using human body as a transmission channel," in IEEE Antennas and Propagation Society Symposium, 2004., vol. 1. IEEE, 2004, pp. 105-108.

[164] K. Ito, M. Takahashi, and K. Fujii, "Transmission mechanism of wearable devices using the human body as a transmission channel," Artech House, pp. 65-92, 2006.

[165] K. Fujii, M. Takahashi, and K. Ito, "Electric field distributions of wearable devices using the human body as a transmission channel," IEEE Trans. Antennas Propag., vol. 55, no. 7, pp. 2080-2087, 2007.

[166] J. Wang, Y. Nishikawa, and T. Shibata, "Analysis of on-body transmission mechanism and characteristic based on an electromagnetic field approach," IEEE Trans. Microw. Theory Techn., vol. 57, no. 10, pp. 2464-2470, 2009.

[167] K. S.-P. Kamya Yekeh Yazdandoost, "Channel model for body area network (ban)," IEEE P802.15-08-0780-12-0006, Nov. 2010.

[168] A. Alomainy and Y. Hao, "Modeling and characterization of biotelemetric radio channel from ingested implants considering organ contents," IEEE Trans. Antennas Propag., vol. 57, no. 4, pp. 999-1005, 2009

[169] B. Zhen, K. Takizawa, T. Aoyagi, and R. Kohno, "A body surface coordinator for implanted biosensor networks," in 2009 IEEE International Conference on Communications, 2009, pp. 1-5.

[170] R. Chávez-Santiago, C. Garcia-Pardo, A. Fornes-Leal, A. VallésLluch, G. Vermeeren, W. Joseph, I. Balasingham, and N. Cardona, "Experimental path loss models for in-body communications within 2.36-2.5 ghz," IEEE journal of biomedical and health informatics, vol. 19, no. 3, pp. 930-937, 2015.

[171] A. Khaleghi, R. Chávez-Santiago, and I. Balasingham, "Ultra-wideband statistical propagation channel model for implant sensors in the human chest," vol. 5, no. 15, pp. 1805-1812, 2011.

[172] K. M. S. Thotahewa, J. Redoutè, and M. R. Yuce, "Propagation, power absorption, and temperature analysis of uwb wireless capsule endoscopy devices operating in the human body," IEEE Trans. Microw. Theory Techn., vol. 63, no. 11, pp. 3823-3833, 2015.

[173] Y. Shimizu, T. Furukawa, D. Anzai, and J. Wang, "Performance improvement by transmit diversity technique for implant ultra-wideband communication," IET Microwaves, Antennas Propagation, vol. 10, no. 10, pp. 1106-1112, 2016.

[174] J. Shi and J. Wang, "Channel characterization and diversity feasibility for in-body to on-body communication using low-band uwb signals," in 2010 3rd International Symposium on Applied Sciences in Biomedical and Communication Technologies (ISABEL 2010), 2010, pp. 1-4.

[175] M. Kanaan and M. Suveren, "A novel frequency-dependent path loss model for ultra wideband implant body area networks," Measurement, vol. 68 , pp. $117-127,2015$

[176] R. A. Receveur, F. W. Lindemans, and N. F. De Rooij, "Microsystem technologies for implantable applications," Journal of Micromechanics and Microengineering, vol. 17, no. 5, p. R50, 2007.

[177] K. Sayrafian, "Mics channel characteristics, preliminary results," IEEE 802.15-08-0351-00-0006, May 2008.

[178] —_ "Ieee 802.15-08-0519-01-0006," A Statistical Path Loss Model for MICS, Sep. 2008.

[179] C. Garcia-Pardo, C. Andreu, A. Fornes-Leal, S. Castelló-Palacios, S. Perez-Simbor, M. Barbi, A. Vallés-Lluch, and N. Cardona, "Ultrawideband technology for medical in-body sensor networks: An overview of the human body as a propagation medium, phantoms, and approaches for propagation analysis," IEEE Antennas and Propagation Magazine, vol. 60, no. 3, pp. 19-33, 2018.

[180] T. Aoyagi et al., "Ieee 802.15-08-0416-04-0006," Channel model for wearable and implantable WBANs, Nov. 2008

[181] G. Dolmans and A. Fort, "Ieee 802.15-08-0418-01-0006," Channel models WBAN-Holst centre/IMEC-NL, Jul. 2008.

[182] E. Reusens, W. Joseph, B. LatrÉ, B. Braem, G. Vermeeren, E. Tanghe, L. Martens, I. Moerman, and C. Blondia, "Characterization of onbody communication channel and energy efficient topology design for wireless body area networks," IEEE Trans. Inf. Tech. Biomed., vol. 13, no. 6, pp. 933-945, Nov. 2009

[183] A. Fort, C. Dessetand P. Wambacq, and L. Biesen, "Indoor bodyarea channel model for narrowband communications," IET Microwaves, Antennas \& Propagation, vol. 1, pp. 1197-1203(6), December 2007. 
[184] A. Fort, C. Desset, J. Ryckaert, P. De Doncker, L. Van Biesen, and P. Wambacq, "Characterization of the ultra wideband body area propagation channel," in 2005 IEEE International Conference on UltraWideband, Sep. 2005.

[185] Y. Chen, J. Teo, J. C. Y. Lai, E. Gunawan, K. S. Low, C. B. Soh, and P. B. Rapajic, "Cooperative communications in ultra-wideband wireless body area networks: Channel modeling and system diversity analysis," IEEE J. Sel. Areas Commun., vol. 27, no. 1, pp. 5-16, 2009.

[186] S. van Roy, F. Quitin, L. Liu, C. Oestges, F. Horlin, J. Dricot, and P. De Doncker, "Dynamic channel modeling for multi-sensor body area networks," IEEE Trans. Antennas Propag., vol. 61, no. 4, pp. 2200 2208, Apr. 2013.

[187] A. Sani, A. Alomainy, G. Palikaras, Y. Nechayev, Y. Hao, C. Parini, and P. S. Hall, "Experimental characterization of uwb on-body radio channel in indoor environment considering different antennas," IEEE Trans. Antennas Propag., vol. 58, no. 1, pp. 238-241, Jan. 2010.

[188] T. Kumpuniemi, T. Tuovinen, M. Hämäläinen, K. Y. Yazdandoost, R. Vuohtoniemi, and J. Iinatti, "Measurement-based on-body path loss modelling for uwb wban communications," in 2013 7th International Symposium on Medical Information and Communication Technology (ISMICT), 2013, pp. 233-237.

[189] A. Fort, C. Desset, P. De Doncker, P. Wambacq, and L. Van Biesen, "An ultra-wideband body area propagation channel model-from statistics to implementation," IEEE Trans. Microw. Theory Techn., vol. 54, no. 4, pp. 1820-1826, 2006.

[190] Y. P. Zhang and Q. Li, "Performance of uwb impulse radio with planar monopoles over on-human-body propagation channel for wireless body area networks," IEEE Trans. Antennas Propag., vol. 55, no. 10, pp. 2907-2914, Oct. 2007.

[191] A. Fort, J. Ryckaert, C. Desset, P. De Doncker, P. Wambacq, and L. Van Biesen, "Ultra-wideband channel model for communication around the human body," IEEE J. Sel. Areas Commun., vol. 24, no. 4, pp. 927-933, Apr. 2006

[192] P. Thirumaraiselvan, K. Sakthidasan, V. B. Geo, V. Prakash, N. Vasudevan et al., "A statistical lower uwb channel model for in body communications," Mobile Networks and Applications, vol. 24, no. 6, pp. 1814-1820, 2019.

[193] M. Mackowiak and L. M. Correia, "Statistical path loss model for dynamic off-body channels," in 2014 IEEE 25th Annual International Symposium on Personal, Indoor, and Mobile Radio Communication (PIMRC), 2014, pp. 53-57.

[194] A. F. Molisch, K. Balakrishnan, D. Cassioli, Chia-Chin Chong, S. Emami, A. Fort, J. Karedal, J. Kunisch, H. Schantz, and K. Siwiak, "A comprehensive model for ultrawideband propagation channels," in GLOBECOM '05. IEEE Global Telecommunications Conference, 2005., vol. 6, Nov. 2005, pp. 6 pp.-3653.

[195] R. Rosini and R. D'Errico, "Off-body channel modelling at $2.45 \mathrm{ghz}$ for two different antennas," in 2012 6th European Conference on Antennas and Propagation (EUCAP), 2012, pp. 3378-3382.

[196] M. Särestöniemi, T. Tuovinen, M. Hämäläinen, K. Y. Yazdandoost, and J. H. Iinatti, "Channel modeling for uwb wban on-off body communication link with finite integration technique." in BODYNETS 2012, pp. 235-241.

[197] P. S. Hall, M. Ricci, and T. M. Hee, "Measurements of on-body propagation characteristics," in IEEE Antennas and Propagation Society International Symposium (IEEE Cat. No.02CH37313), vol. 2, 2002, pp. 310-313 vol.2.

[198] D. B. Smith, L. W. Hanlen, J. A. Zhang, D. Miniutti, D. Rodda, and B. Gilbert, "First- and second-order statistical characterizations of the dynamic body area propagation channel of various bandwidths," Annals of Telecommunications - annales des télécommunications, vol. 66, no. 3, pp. 187-203, 2011.

[199] T. Zasowski, F. Althaus, M. Stager, A. Wittneben, and G. Troster, "Uwb for noninvasive wireless body area networks: channel measurements and results," in IEEE Conference on Ultra Wideband Systems and Technologies, 2003, Nov. 2003, pp. 285-289.

[200] Y. I. Nechayev, P. S. Hall, I. Khan, and C. C. Constantinou, "Wireless channels and antennas for body-area networks," in 2010 Seventh International Conference on Wireless On-demand Network Systems and Services (WONS), 2010, pp. 137-144.

[201] D. B. Smith, D. Miniutti, L. W. Hanlen, D. Rodda, and B. Gilbert, "Dynamic narrowband body area communications: Link-margin based performance analysis and second-order temporal statistics," in 2010 IEEE Wireless Communication and Networking Conference, 2010, pp. $1-6$.

[202] D. B. Smith, T. Lamahewa, L. W. Hanlen, and D. Miniutti, "Simple prediction-based power control for the on-body area communications channel," in 2011 IEEE International Conference on Communications (ICC), 2011, pp. 1-5.

[203] P. Sroka and J. A. Samuels, “Adaptive antenna matching," Jul. 7 1998, uS Patent 5,778,308.

[204] P. W. Dent and R. A. Dolman, "System and method for adaptive antenna impedance matching," Jan. 18 2005, uS Patent 6,845,126.

[205] P. W. Dent and S. Mattisson, "System and method for adaptive antenna impedance matching," Jan. 8 2013, uS Patent 8,351,874.

[206] C. Roblin, J.-M. Laheurte, R. D'Errico, A. Gati, D. Lautru, T. Alvès, H. Terchoune, and F. Bouttout, "Antenna design and channel modeling in the ban context-part i: Antennas," Annals of Telecommunicationsannales des télécommunications, vol. 66, no. 3-4, pp. 139-155, 2011.

[207] S. Yan, P. J. Soh, and G. A. E. Vandenbosch, "Wearable ultrawideban technology - a review of ultrawideband antennas, propagation channels, and applications in wireless body area networks," IEEE Access, vol. 6 , pp. $42177-42185,2018$

[208] T. G. Zimmerman, "Personal area networks: Near-field intrabody communication," IBM Systems Journal, vol. 35, no. 3.4, pp. 609-617, 1996.

[209] T. Handa, S. Shoji, S. Ike, S. Takeda, and T. Sekiguchi, "A very lowpower consumption wireless ecg monitoring system using body as a signal transmission medium," in Proceedings of International Solid State Sensors and Actuators Conference (Transducers' 97), vol. 2. IEEE, 1997, pp. 1003-1006.

[210] T. Ogasawara, A.-i. Sasaki, K. Fujii, and H. Morimura, "Human body communication based on magnetic coupling," IEEE Trans. Antennas Propag., vol. 62, no. 2, pp. 804-813, 2013.

[211] M. S. Wegmueller, M. Oberle, N. Felber, N. Kuster, and W. Fichtner, "Signal transmission by galvanic coupling through the human body," IEEE Trans. Instrum. Meas., vol. 59, no. 4, pp. 963-969, 2009.

[212] J. Bae, H. Cho, K. Song, H. Lee, and H.-J. Yoo, "The signal transmission mechanism on the surface of human body for body channel communication," IEEE Trans. Microw. Theory Techn., vol. 60, no. 3 , pp. 582-593, 2012.

[213] A. K. Teshome, B. Kibret, and D. T. H. Lai, "Galvanically coupled intrabody communications for medical implants: A unified analytic model," IEEE Trans. Antennas Propag., vol. 64, no. 7, pp. 2989-3002, 2016.

[214] N. Cho, J. Yoo, S.-J. Song, J. Lee, S. Jeon, and H.-J. Yoo, "The human body characteristics as a signal transmission medium for intrabody communication," IEEE Trans. Microw. Theory Techn., vol. 55, no. 5, pp. 1080-1086, 2007.

[215] Y. Song, Q. Hao, K. Zhang, M. Wang, Y. Chu, and B. Kang, "The simulation method of the galvanic coupling intrabody communication with different signal transmission paths," IEEE Trans. Instrum. Meas., vol. 60, no. 4, pp. 1257-1266, 2010.

[216] M. A. Callejon, D. Naranjo-Hernandez, J. Reina-Tosina, and L. M. Roa, "Distributed circuit modeling of galvanic and capacitive coupling for intrabody communication," IEEE Trans. Biomed. Eng., vol. 59, no. 11, pp. 3263-3269, 2012.

[217] B. Kibret, M. Seyedi, D. T. Lai, and M. Faulkner, "Investigation of galvanic-coupled intrabody communication using the human body circuit model," IEEE Journal of Biomedical and Health Informatics, vol. 18, no. 4, pp. 1196-1206, 2014.

[218] M. Pereira, G. Alvarez, and F. de Sousa, "Modeling of the test fixtures to improve the hbc channel interpretation," in 2015 IEEE International Instrumentation and Measurement Technology Conference (I2MTC) Proceedings. IEEE, 2015, pp. 1753-1756.

[219] M. D. Pereira, G. A. Alvarez-Botero, and F. R. de Sousa, "Characterization and modeling of the capacitive hbc channel," IEEE Trans. Instrum. Meas., vol. 64, no. 10, pp. 2626-2635, 2015.

[220] M. Swaminathan, F. S. Cabrera, J. S. Pujol, U. Muncuk, G. Schirner, and K. R. Chowdhury, "Multi-path model and sensitivity analysis for galvanic coupled intra-body communication through layered tissue," IEEE Trans. Biomed. Syst., vol. 10, no. 2, pp. 339-351, 2016.

[221] J. Mao, H. Yang, Y. Lian, and B. Zhao, "A five-tissue-layer human body communication circuit model tunable to individual characteristics," IEEE Trans. Biomed. Circuits Syst., vol. 12, no. 2, pp. 303-312, 2018.

[222] S. Maity, M. He, M. Nath, D. Das, B. Chatterjee, and S. Sen, "Bio-physical modeling, characterization, and optimization of electroquasistatic human body communication," IEEE Trans. Biomed. Eng., vol. 66, no. 6, pp. 1791-1802, 2019.

[223] I. N. Alquaydheb, A. E. Khorshid, and A. M. Eltawil, "Analysis and estimation of intra-body communications path loss for galvanic coupling," in Advances in Body Area Networks I - Technology, Communications and Computing, G. Fortino and Z. Wang, Eds. 
Springer, 2019, pp. 267-277. [Online]. Available: https://doi.org/10. 1007/978-3-030-02819-0_20

[224] J. Park, H. Garudadri, and P. P. Mercier, "Channel modeling of miniaturized battery-powered capacitive human body communication systems," IEEE Trans. Biomed. Eng., vol. 64, no. 2, pp. 452-462, 2016.

[225] Ž. Lucev, I. Krois, and M. Cifrek, "A capacitive intrabody communication channel from $100 \mathrm{khz}$ to $100 \mathrm{mhz}$," IEEE Trans. Instrum. Meas., vol. 61 , no. 12, pp. 3280-3289, 2012.

[226] J. Hwang, T. Kang, Y. Kim, and S. Park, "Measurement of transmission properties of hbc channel and its impulse response model," IEEE Trans. Instrum. Meas., vol. 65, no. 1, pp. 177-188, 2016.

[227] Y. Liu, J. Kuang, Z. He, and J. Fang, "Measurement system for propagation characteristics of intra-body communication," in 2010 6th International Conference On Wireless Communications Networking And Mobile Computing (WiCOM). IEEE, 2010, pp. 1-4.

[228] J. A. Ruiz and S. Shimamoto, "Experimental evaluation of body channel response and digital modulation schemes for intra-body communications," in 2006 IEEE International Conference on Communications, vol. 1. IEEE, 2006, pp. 349-354.

[229] J. A. Ruiz, J. Xu, and S. Shimamoto, "Propagation characteristics of intra-body communications for body area networks," in CCNC 2006. 2006 3rd IEEE Consumer Communications and Networking Conference, 2006., vol. 1. IEEE, 2006, pp. 509-513.

[230] R. Xu, H. Zhu, and J. Yuan, "Characterization and analysis of intrabody communication channel," in 2009 IEEE Antennas and Propagation Society International Symposium. IEEE, 2009, pp. 1-4.

[231] W.-c. Wang, Z.-d. Nie, F. Guan, T.-f. Leng, and L. Wang, "Experimental studies on human body communication characteristics based upon capacitive coupling," in 2011 International Conference on Body Sensor Networks. IEEE, 2011, pp. 180-185.

[232] T. C. Schenk, N. S. Mazloum, L. Tan, and P. Rutten, "Experimental characterization of the body-coupled communications channel," in 2008 IEEE International Symposium on Wireless Communication Systems. IEEE, 2008, pp. 234-239.

[233] N. Zedong, M. Jingjing, K. Ivanov, and W. Lei, "An investigation on dynamic human body communication channel characteristics at $45 \mathrm{mhz}$ in different surrounding environments," IEEE Antennas and Wireless Propagation Letters, vol. 13, pp. 309-312, 2014.

[234] M. A. Callejón, J. Reina-Tosina, D. Naranjo-Hernández, and L. M. Roa, "Measurement issues in galvanic intrabody communication: Influence of experimental setup," IEEE Trans. Biomed. Eng., vol. 62, no. 11, pp. 2724-2732, 2015.

[235] G. A. Álvarez-Botero, Y. K. Hernández-Gómez, C. E. Telléz, and J. F. Coronel, "Human body communication: Channel characterization issues," IEEE Instrumentation \& Measurement Magazine, vol. 22, no. 5, pp. 48-53, 2019.

[236] M. A. Callejon, D. Naranjo-Hernandez, J. Reina-Tosina, and L. M. Roa, "A comprehensive study into intrabody communication measurements," IEEE Trans. Instrum. Meas., vol. 62, no. 9, pp. 2446-2455, 2013.

[237] F. Lu, C. Wang, R. Zhao, L. Du, Z. Fang, X. Guo, and Z. Zhao, "Review of stratum corneum impedance measurement in non-invasive penetration application," Biosensors, vol. 8, no. 2, p. 31, 2018

[238] A. E. Khorshid, I. N. Alquaydheb, and A. M. Eltawil, "Electrode impedance modeling for channel characterization for intra-body communication," in Advances in Body Area Networks I - Technology, Communications and Computing, G. Fortino and Z. Wang, Eds. Springer, 2019, pp. 253-266. [Online]. Available: https://doi.org/10. 1007/978-3-030-02819-0_19

[239] F. Xiong, D. Chen, Z. Chen, C. Jin, and S. Dai, "Impedance characteristics of the skin-electrode interface of dry textile electrodes for wearable electrocardiogram," in Advances in Body Area Networks I, G. Fortino and Z. Wang, Eds. Cham: Springer International Publishing, 2019, pp. 343-356.

[240] A. C. Ahn and Ø. G. Martinsen, "Electrical characterization of acupuncture points: technical issues and challenges," The journal of alternative and complementary medicine, vol. 13, no. 8, pp. 817-824, 2007

[241] H.-J. Yoo and C. van Hoof, Bio-Medical CMOS ICs. Springer, 2010.

[242] S. Schwingenschuh, H. Scharfetter, Ø. G. Martinsen, B. Boulgaropoulos, T. Augustin, K. I. Tiffner, C. Dragatin, R. Raml, C. Hoefferer, E.C. Prandl et al., "Assessment of skin permeability to topically applied drugs by skin impedance and admittance," Physiological measurement, vol. 38, no. 11, p. N138, 2017.

[243] Y. Meng, Z. Li, and J. Chen, "A flexible dry electrode based on aptes-anchored pdms substrate for portable ecg acquisition system," Microsystem Technologies, vol. 22, no. 8, pp. 2027-2034, 2016.
[244] A. J. Bandodkar, W. Jia, C. Yardımc1, X. Wang, J. Ramirez, and J. Wang, "Tattoo-based noninvasive glucose monitoring: a proof-ofconcept study," Analytical chemistry, vol. 87, no. 1, pp. 394-398, 2015.

[245] Y. Chen, S. Lu, S. Zhang, Y. Li, Z. Qu, Y. Chen, B. Lu, X. Wang, and $X$. Feng, "Skin-like biosensor system via electrochemical channels for noninvasive blood glucose monitoring," Science advances, vol. 3 , no. 12, p. e1701629, 2017.

[246] S. Yao and Y. Zhu, "Nanomaterial-enabled dry electrodes for electrophysiological sensing: A review," Jom, vol. 68, no. 4, pp. 1145-1155, 2016.

[247] C.-u. Kim, G. Li, J. Li, H. Jong, C. Ro, Y. Song, G. Pak, and S. Im, "Numerical analysis on effective electric field penetration depth for interdigital impedance sensor," in J. Phys. Conf. Ser, vol. 418, no. 1, 2013, p. 012020

[248] M. Varshney and Y. Li, "Interdigitated array microelectrodes based impedance biosensors for detection of bacterial cells," Biosensors and bioelectronics, vol. 24, no. 10, pp. 2951-2960, 2009.

[249] B. Zhao, J. Mao, J. Zhao, H. Yang, and Y. Lian, "The role and challenges of body channel communication in wearable flexible electronics," IEEE Trans. Biomed. Circuits Syst., vol. 14, no. 2, pp. $283-$ 296, 2020

[250] H. Wang, X. Tang, C. S. Choy, and G. E. Sobelman, "Cascaded network body channel model for intrabody communication," IEEE Journal of Biomedical and Health Informatics, vol. 20, no. 4, pp. 1044-1052, 2016.

[251] B. Zhao, Y. Lian, A. M. Niknejad, and C. H. Heng, "A low-power compact ieee 802.15. 6 compatible human body communication transceiver with digital sigma-delta iir mask shaping," IEEE Journal of Solid-State Circuits, vol. 54, no. 2, pp. 346-357, 2018.

[252] J. Bae, K. Song, H. Lee, H. Cho, and H.-J. Yoo, "A 0.24-nj/b wireless body-area-network transceiver with scalable double-fsk modulation," IEEE Journal of Solid-State Circuits, vol. 47, no. 1, pp. 310-322, 2011.

[253] Y. L. Tsou, C.-S. A. Gong, N. C. Cheng, Y. Lee, and C. F. Jou, "Integrated biosensing platform based on a 1.74-mw- $90-\mathrm{dbm}$ sensitivity dual-mode-operation receiver for ieee 802.15. 6 human body communication standard," IEEE Sensors Journal, vol. 15, no. 6, pp. 3317-3327, 2014.

[254] K.-R. Lee, J. Jang, H. Cho, and H.-J. Yoo, "A $635 \mu$ w non-contact compensation ic for body channel communication," in 2016 IEEE International Symposium on Circuits and Systems (ISCAS). IEEE, 2016, pp. 1406-1409.

[255] J. Zhao, J. Mao, T. Zhou, L. Lai, H. Yang, and B. Zhao, "An auto loss compensation system for non-contact capacitive coupled body channel communication," in 2018 IEEE International Symposium on Circuits and Systems (ISCAS). IEEE, 2018, pp. 1-5.

[256] Y. Liu, Z. Tan, H. Hu, L. J. Cimini, and G. Y. Li, "Channel estimation for ofdm," IEEE Communications Surveys Tutorials, vol. 16, no. 4, pp. 1891-1908, 2014.

[257] M. Morelli and U. Mengali, "A comparison of pilot-aided channel estimation methods for ofdm systems," IEEE Trans. Signal Process., vol. 49, no. 12, pp. 3065-3073, 2001.

[258] M. C. Valenti and B. D. Woerner, "Iterative channel estimation and decoding of pilot symbol assisted turbo codes over flat-fading channels," IEEE J. Sel. Areas Commun., vol. 19, no. 9, pp. 1697-1705, 2001.

[259] H. Ye, G. Y. Li, and B. Juang, "Power of deep learning for channel estimation and signal detection in ofdm systems," IEEE Wireless Commun. Lett., vol. 7, no. 1, pp. 114-117, 2018.

[260] H. Huang, J. Yang, H. Huang, Y. Song, and G. Gui, "Deep learning for super-resolution channel estimation and doa estimation based massive mimo system," IEEE Trans. Veh. Technol., vol. 67, no. 9, pp. 85498560, 2018.

[261] A. Zappone, M. Di Renzo, and M. Debbah, "Wireless networks design in the era of deep learning: Model-based, ai-based, or both?" IEEE Trans. Commun., vol. 67, no. 10, pp. 7331-7376, 2019.

[262] Xiaojun Lin, N. B. Shroff, and R. Srikant, "A tutorial on crosslayer optimization in wireless networks," IEEE J. Sel. Areas Commun., vol. 24, no. 8, pp. 1452-1463, 2006.

[263] V. Leonov, P. Fiorini, and R. J. Vullers, "Theory and simulation of a thermally matched micromachined thermopile in a wearable energy harvester," Microelectronics Journal, vol. 42, no. 4, pp. 579-584, 2011.

[264] V. Leonov, "Thermoelectric energy harvesting of human body heat for wearable sensors," IEEE Sensors Journal, vol. 13, no. 6, pp. 22842291, 2013.

[265] T. Von Buren, P. D. Mitcheson, T. C. Green, E. M. Yeatman, A. S Holmes, and G. Troster, "Optimization of inertial micropower generators for human walking motion," IEEE Sensors journal, vol. 6, no. 1, pp. 28-38, 2006. 
[266] C. Saha, T. O'donnell, N. Wang, and P. McCloskey, "Electromagnetic generator for harvesting energy from human motion," Sensors and Actuators A: Physical, vol. 147, no. 1, pp. 248-253, 2008.

[267] H. Yetgin, K. T. K. Cheung, M. El-Hajjar, and L. H. Hanzo, "A survey of network lifetime maximization techniques in wireless sensor networks," IEEE Commun. Surveys Tuts., vol. 19, no. 2, pp. 828-854, 2017.

[268] X. Liu, E. K. P. Chong, and N. B. Shroff, "Opportunistic transmission scheduling with resource-sharing constraints in wireless networks," IEEE J. Sel. Areas Commun., vol. 19, no. 10, pp. 2053-2064, 2001.

[269] B. Prabhakar, E. U. Biyikoglu, and A. El Gamal, "Energy-efficient transmission over a wireless link via lazy packet scheduling," in Proceedings IEEE INFOCOM 2001. Conference on Computer Communications. Twentieth Annual Joint Conference of the IEEE Computer and Communications Society (Cat. No. O1CH37213), vol. 1. IEEE, 2001, pp. 386-394.

[270] D. L. Donoho, "Compressed sensing," IEEE Trans. Inf. Theory, vol. 52, no. 4, pp. 1289-1306, 2006.

[271] H. Mamaghanian, N. Khaled, D. Atienza, and P. Vandergheynst, "Compressed sensing for real-time energy-efficient ECG compression on wireless body sensor nodes," IEEE Trans. Biomed. Eng., vol. 58, no. 9, pp. 2456-2466, 2011.

[272] Z. Zhang, T.-P. Jung, S. Makeig, and B. D. Rao, "Compressed sensing of EEG for wireless telemonitoring with low energy consumption and inexpensive hardware," IEEE Trans. Biomed. Eng., vol. 60, no. 1, pp. 221-224, 2012.

[273] A. M. Dixon, E. G. Allstot, D. Gangopadhyay, and D. J. Allstot, "Compressed sensing system considerations for ECG and EMG wireless biosensors," IEEE Trans. Biomed. Circuits Syst., vol. 6, no. 2, pp. 156166, 2012.

[274] S. A. Gopalan and J.-T. Park, "Energy-efficient mac protocols for wireless body area networks: Survey," in International Congress on Ultra Modern Telecommunications and Control Systems. IEEE, 2010, pp. 739-744.

[275] S. Marinkovic, C. Spagnol, and E. Popovici, "Energy-efficient tdmabased mac protocol for wireless body area networks," in 2009 Third International Conference on Sensor Technologies and Applications. IEEE, 2009, pp. 604-609.

[276] G. Bianchi, L. Fratta, and M. Oliveri, "Performance evaluation and enhancement of the csma/ca mac protocol for 802.11 wireless lans," in Proceedings of PIMRC'96-7th International Symposium on Personal, Indoor, and Mobile Communications, vol. 2. IEEE, 1996, pp. 392-396.

[277] X. Wang and K. Kar, "Throughput modelling and fairness issues in csma/ca based ad-hoc networks," in Proceedings IEEE 24th Annual Joint Conference of the IEEE Computer and Communications Societies., vol. 1. IEEE, 2005, pp. 23-34.

[278] T. R. Park, T. H. Kim, J. Y. Choi, S. Choi, and W. H. Kwon, "Throughput and energy consumption analysis of ieee 802.15. 4 slotted csma/ca," Electronics Letters, vol. 41, no. 18, pp. 1017-1019, 2005.

[279] M. Särestöniemi, M. Hämäläinen, and J. Iinatti, "An overview of the electromagnetic simulation-based channel modeling techniques for wireless body area network applications," IEEE Access, vol. 5, pp. 10622-10632, 2017.

[280] A. Bachir, M. Dohler, T. Watteyne, and K. K. Leung, "Mac essentials for wireless sensor networks," IEEE Commun. Surveys Tuts., vol. 12, no. 2, pp. 222-248, 2010.

[281] T. Van Dam and K. Langendoen, "An adaptive energy-efficient mac protocol for wireless sensor networks," in Proceedings of the 1st international conference on Embedded networked sensor systems, 2003, pp. 171-180.

[282] T. Wu, T. S. Rappaport, and C. M. Collins, "The human body and millimeter-wave wireless communication systems: Interactions and implications," in 2015 IEEE International Conference on Communications (ICC), 2015, pp. 2423-2429.

[283] M. Li, W. Lou, and K. Ren, "Data security and privacy in wireless body area networks," IEEE Wireless Commun., vol. 17, no. 1, pp. 5158, 2010.

[284] S. Maity, M. He, M. Nath, D. Das, B. Chatterjee, and S. Sen, "Bio-physical modeling, characterization, and optimization of electroquasistatic human body communication," IEEE Trans. Biomed. Eng., vol. 66, no. 6, pp. 1791-1802, 2018.

[285] D. Das, S. Maity, B. Chatterjee, and S. Sen, "Enabling covert body area network using electro-quasistatic human body communication," Scientific reports, vol. 9, no. 1, pp. 1-14, 2019. 